\title{
Forelimb muscle and joint actions in Archosauria: insights from Crocodylus johnstoni (Pseudosuchia) and Mussaurus patagonicus (Sauropodomorpha)
}

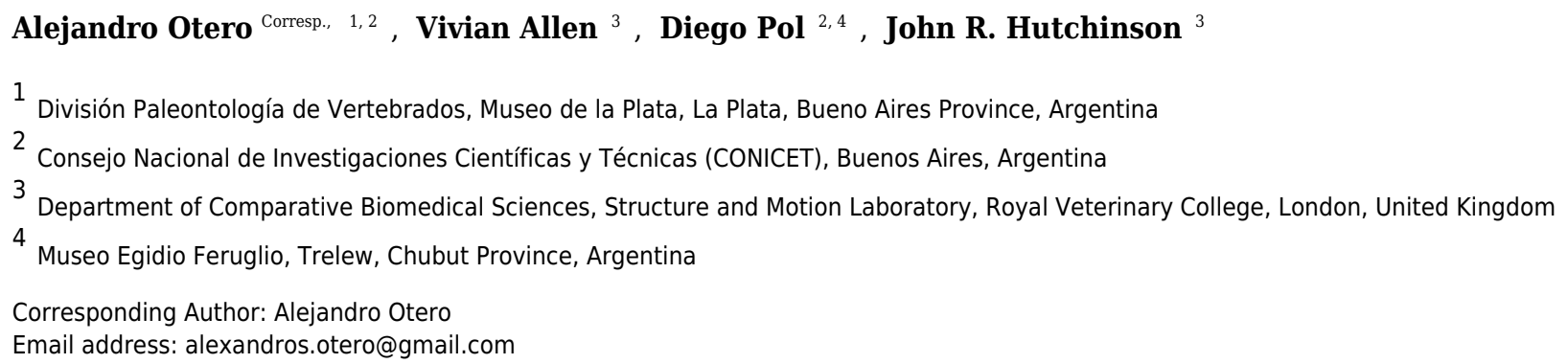

Many of the major locomotor transitions during the evolution of Archosauria, the lineage including crocodiles and birds as well as extinct Dinosauria, were shifts from quadrupedalism to bipedalism (and vice versa). Those occurred within a continuum between more sprawling and erect modes of locomotion and involved drastic changes of limb anatomy and function in several lineages, including sauropodomorph dinosaurs. We present biomechanical computer models of two locomotor extremes within Archosauria in an analysis of joint ranges of motion and the moment arms of the major forelimb muscles in order to quantify biomechanical differences between more sprawling, pseudosuchian (represented the crocodile Crocodylus johnstoni) and more erect, dinosaurian (represented by the sauropodomorph Mussaurus patagonicus) modes of forelimb function. We compare these two locomotor extremes in terms of the reconstructed musculoskeletal anatomy, ranges of motion of the forelimb joints and the moment arm patterns of muscles across those ranges of joint motion. We reconstructed the three-dimensional paths of 31 muscles acting around the shoulder, elbow and wrist joints. We explicitly evaluate how forelimb joint mobility and muscle actions may have changed with postural and anatomical alterations from basal archosaurs to early sauropodomorphs. We thus evaluate in which ways forelimb posture was correlated with muscle leverage, and how such differences fit into a broader evolutionary context (i.e. transition from sprawling quadrupedalism to erect bipedalism and then shifting to graviportal quadrupedalism). Our analysis reveals major differences of muscle actions between the more sprawling and erect models at the shoulder joint. These differences are related not only to the articular surfaces but also to the orientation of the scapula, in which extension/flexion movements in Crocodylus (e.g. protraction of the humerus) correspond to elevation/depression in Mussaurus. Muscle action is highly influenced by limb posture, more so than morphology. Habitual 
quadrupedalism in Mussaurus is not supported by our analysis of joint range of motion, which indicates that glenohumeral protraction was severely restricted. Additionally, some active pronation of the manus may have been possible in Mussaurus, allowing semipronation by a rearranging of the whole antebrachium (not the radius against the ulna, as previously thought) via long-axis rotation at the elbow joint. However, the muscles acting around this joint to actively pronate it may have been too weak to drive or maintain such orientations as opposed to a neutral position in between pronation and supination. Regardless, the origin of quadrupedalism in Sauropoda is not only linked to manus pronation but also to multiple shifts of forelimb morphology, allowing greater flexion movements of the glenohumeral joint and a more columnar forelimb posture. 
1 Forelimb muscle and joint actions in Archosauria: insights from Crocodylus johnstoni

2 (Pseudosuchia) and Mussaurus patagonicus (Sauropodomorpha)

3

4 Alejandro Otero ${ }^{1,2 *}$, Vivian Allen ${ }^{3}$, Diego Pol ${ }^{4,2}$, John R. Hutchinson ${ }^{3}$

5

$6 \quad$ 1División Paleontología de Vertebrados, Museo de La Plata, Paseo del Bosque s/n, La Plata

7 (1900), Argentina.

$8 \quad{ }^{2}$ CONICET - Consejo Nacional de Investigaciones Científicas y Técnicas.

9 3Structure and Motion Laboratory, Department of Comparative Biomedical Sciences, The Royal

10 Veterinary College, Hatfield, Hertfordshire, United Kingdom.

$11{ }^{4}$ Museo Paleontológico Egidio Feruglio, Trelew, Argentina.

12

13

* Correspondence to: alexandros.otero@gmail.com

14

15 
ABSTRACT:

Many of the major locomotor transitions during the evolution of Archosauria, the lineage including crocodiles and birds as well as extinct Dinosauria, were shifts from quadrupedalism to bipedalism (and vice versa). Those occurred within a continuum between more sprawling and erect modes of locomotion and involved drastic changes of limb anatomy and function in several lineages, including sauropodomorph dinosaurs. We present biomechanical computer models of two locomotor extremes within Archosauria in an analysis of joint ranges of motion and the moment arms of the major forelimb muscles in order to quantify biomechanical differences between more sprawling, pseudosuchian (represented the crocodile Crocodylus johnstoni) and more erect, dinosaurian (represented by the sauropodomorph Mussaurus patagonicus) modes of forelimb function. We compare these two locomotor extremes in terms of the reconstructed musculoskeletal anatomy, ranges of motion of the forelimb joints and the moment arm patterns of muscles across those ranges of joint motion. We reconstructed the three-dimensional paths of 31 muscles acting around the shoulder, elbow and wrist joints. We explicitly evaluate how forelimb joint mobility and muscle actions may have changed with postural and anatomical alterations from basal archosaurs to early sauropodomorphs. We thus evaluate in which ways forelimb posture was correlated with muscle leverage, and how such differences fit into a broader evolutionary context (i.e. transition from sprawling quadrupedalism to erect bipedalism and then shifting to graviportal quadrupedalism). Our analysis reveals major differences of muscle actions between the more sprawling and erect models at the shoulder joint. These differences are related not only to the articular surfaces but also to the orientation of the scapula, in which extension/flexion movements in Crocodylus (e.g. 
38 protraction of the humerus) correspond to elevation/depression in Mussaurus. Muscle action is

39 highly influenced by limb posture, more so than morphology. Habitual quadrupedalism in

40 Mussaurus is not supported by our analysis of joint range of motion, which indicates that

41 glenohumeral protraction was severely restricted. Additionally, some active pronation of the

42 manus may have been possible in Mussaurus, allowing semi-pronation by a rearranging of the

43 whole antebrachium (not the radius against the ulna, as previously thought) via long-axis

44 rotation at the elbow joint. However, the muscles acting around this joint to actively pronate it

45 may have been too weak to drive or maintain such orientations as opposed to a neutral

46 position in between pronation and supination. Regardless, the origin of quadrupedalism in

47 Sauropoda is not only linked to manus pronation but also to multiple shifts of forelimb

48 morphology, allowing greater flexion movements of the glenohumeral joint and a more

49 columnar forelimb posture. 


\section{INTRODUCTION}

52 Archosauria (all descendants of the most recent common ancestor of Crocodylia and Aves) has

53

54

been a highly diverse and disparate clade since the Triassic period ( $<250 \mathrm{Ma}$ ), both

morphologically and ecologically. This diversity and disparity is reflected not only in the great abundance and taxonomic richness that Archosauria achieved in the past, but also in its living representatives. Terrestrial locomotion in extant archosaurs (crocodiles and birds) is split between two extremes - 'sprawling' (less erect; Gatesy, 1991) quadrupeds and parasagitally erect bipeds (not to mention amphibious habits vs. flight). The evolutionary patterns that preceded and gave rise to these disparities have long been an attractive research subject (e.g. Romer, 1956; Jenkins, 1993; Gatesy \& Middleton, 1997; Hutchinson \& Allen, 2009; Gauthier et al., 2011; Bates \& Schachner, 2012), including the study of topics such as the transition from bipedalism to quadrupedalism (e.g. Bonnan \& Yates, 2007; Maidment \& Barrett, 2011), and the origin of avian flight (e.g. Jenkins, 1993; Dial, 2003), among others.

The functional anatomy of these locomotor transitions has also attracted considerable research effort (Romer, 1956; Jenkins, 1993; Gatesy \& Middleton, 1997; Hutchinson \& Allen, 2009; Gauthier et al., 2011; Bates \& Schachner, 2012). Much of the attention has focused on the evolution of the hindlimb in Dinosauriformes as it adapted to the demands of bipedal locomotion (Romer, 1923; Carrano, 2000; Hutchinson \& Gatesy, 2000; Hutchinson et al., 2005; Bates \& Schachner, 2012), particularly in the theropod lineage (e.g. Gatesy, 1990). However, archosaur forelimbs have also undergone major functional transformations in Archosauria. Fewer studies have dealt with changes in forelimb function during the quadruped (e.g., basal archosaurs) to biped (e.g., basal sauropodomorphs) transition (but see Hutson \& Hutson, 2013, 
2014, 2017; Hutson, 2015). The lineage of Triassic archosaurs leading to sauropods began as quadrupeds, transitioned to bipedality close to the base of Dinosauria, and then shifted back to quadrupedality close to or at the base of Sauropoda (Wilson \& Sereno, 1998; Carrano, 2005). The evolution of bipedalism itself has been a rare event, and such reversion to quadrupedalism from bipedalism is extremely rare, with known examples confined exclusively to the Dinosauria: sauropods themselves, and independently in three branches of ornithischian dinosaurs (ceratopsians, ornithopods and thyreophorans [Carrano, 1998; Brusatte et al., 2010; Maidment \& Barrett, 2012; VanBuren \& Bonnan, 2013; Hutson, 2015]).

Along the archosaur lineage leading to sauropods, forelimbs thus evolved from a role as weight-bearing locomotor modules to a variety of grasping and manipulating functions, before re-evolving weight-bearing and locomotor capacity with the transition back to quadrupedalism (e.g., Cooper, 1981; Bonnan \& Senter, 2007, Bonnan \& Yates, 2007; Yates et al., 2010; VanBuren \& Bonnan, 2013). The biped-quadruped transition occurred between basal sauropodomorphs and basal sauropods, near the boundary of the Triassic and Jurassic periods (ca. $200 \mathrm{Ma}$ ). Consequently, the forelimbs of basal sauropodomorphs have captured the attention of palaeontologists because their functional morphology was likely pivotal to the acquisition of quadrupedalism (Bonnan \& Yates, 2007). Previous studies of the anatomical and functional evolution of archosaur forelimbs have focused on reconstructing their general role in locomotion (e.g. Ostrom, 1974; Cooper, 1981; Johnson \& Ostrom, 1995; Dodson \& Farlow, 1997; Paul \& Christiansen, 2000; Schwarz, Frey \& Meyer, 2007; Bonnan \& Senter, 2007; Bonnan \& Yates, 2007; Maidment \& Barrett, 2012; Fujiwara \& Hutchinson, 2012; Baier \& Gatesy, 2013). Recently, studies have begun to focus on the evolution of manual pronation across the biped- 
95

96

97

98

99

100

101

102

103

104

105

106

107

108

109

110

111

112

113

114

115

quadruped transition (Bonnan \& Yates, 2007; VanBuren \& Bonnan, 2013; Hutson, 2015). A

more fully pronated manus (i.e. the palm facing ventrally/caudally) was hypothesised to be necessary to effectively produce braking or propulsive forces at the manus-ground interface (e.g., Bonnan \& Yates, 2007).

Considering that the ancestral condition of the manus in bipedal dinosaurs appears to have been more supinated, with palms that faced medially (i.e. $\sim 90^{\circ}$ from fully supinated) rather than caudally, the evolution of a fully pronated manus is thought to have been crucial to the origin of quadrupedalism in both sauropods and ornithischians (see references above). In particular, the degree to which the the morphology of the ulna and radius (antebrachium; i.e.

forearm) would have permitted pronation in basal sauropodomorphs, and if so how widespread this ability was across the group, remain crucial questions in understanding the evolution of sauropod locomotion. However, the timing and sequence of changes in the functional anatomy of the forelimbs that were involved in the evolution of sauropod locomotion remain unclear, partly because to date the biomechanical factors involved have largely been analysed using only qualitative, two-dimensional methods (but see Reiss \& Mallison, 2014).

Here, we use three-dimensional biomechanical computer models to quantitatively analyse the evolution of forelimb anatomy and function from early archosaurs to later sauropodomorphs. We model an adult Mussaurus patagonicus Bonaparte \& Vince, 1979, a well-preserved representative basal sauropodiform (sensu Sereno, 2007), close to the origin of Sauropoda (Otero \& Pol, 2013; Otero et al., 2015), and the extant Australian freshwater crocodile, Crocodylus johnstoni Krefft, 1873, a long-tailed quadruped reasonably representative 
116 of the ancestral archosaurian condition (Parrish, 1986; Gatesy, 1990). The phylogenetic

117 relationship between Crocodylus and Mussaurus is presented in Figure 1.

118 As in prior studies of archosaurian hind limbs (e.g. Hutchinson et al., 2005; Hutchinson

119 et al., 2008; Bates, Benson \& Falkingham, 2012; Bates \& Schachner, 2012; Maidment \& Barrett,

120 2012), we use these musculoskeletal models to analyse the relationship between joint angles

121 and moment arms for the muscles of the limb (e.g. An et al., 1984; Murray, Delp \& Buchanan,

122 1995; Holzbaur, Murray \& Delp, 2005), as well as possible joint ranges of motion (e.g. Reiss \&

123 Mallison, 2014). By quantifying similarities and differences in estimated limb biomechanical

124 properties from our Crocodylus and Mussaurus models, we can explicitly evaluate how forelimb

125 muscle actions and joint mobility may have changed with posture from basal archosaurs to

126 early sauropodomorphs. 'Action' here is used as a shorthand term for moment arms about

127 particular joints; distinguished from 'function' which would ideally involve broader data such as

128 muscle force output, length change, etc. (e.g. Zajac, 1989; Allen et al., 2014). Our analysis

129 considers these key questions: (1) How did forelimb musculoskeletal anatomy evolve between

130 early, quadrupedal archosaurs (approximated by Crocodylus) and early sauropodomorphs such

131 as Mussaurus? (2) How did this alter muscle action and joint ranges of motion? Particularly, was

132 forelimb pronation possible in early sauropodomorphs like Mussaurus? (3) What were the

133 consequences of 1) and 2) for forelimb posture and function? Particularly, how many observed

134 functional changes helped or hindered the use of the forelimbs in terrestrial locomotion? Might

135 such changes relate to the transitions from sprawling quadrupedalism to erect bipedalism in

136 dinosaurs, and/or to the subequent evolution of graviportal quadrupedalism in sauropods? 

least facultatively) bipedal archosaur. We synthesize our findings with a review and metaanalysis of research on the biped-quadruped transition in sauropodomorphs.

\section{MATERIALS AND METHODS}

\section{Digitization and Musculoskeletal Modelling}

Our model-building procedure for Mussaurus and Crocodylus comprised five steps: (1) bone geometry acquisition, (2) joint axis estimation, (3) muscle reconstruction (Mussaurus only), (4)

muscle path specification (using the results from the prior three steps), (5) joint range of motion analysis, and (6) analysis of muscle moment arms (automatically calculated from the muscle paths by the modelling software [see Delp \& Loan, 2000]).

\section{Bone geometry acquisition}

The remains of the basal sauropodomorph Mussaurus patagonicus comprise several specimens of different ontogenetic stages, from post-hatchlings to adults (Bonaparte \& Vince, 1979; Otero, Pol \& Powell, 2012; Otero \& Pol, 2013). Our study here focused on the best-preserved and complete right forelimb of the adult specimen number MLP 68-II-27-1 (Museo de la Plata,

157 phalanx of digit two (Otero \& Pol, 2013). A three-dimensional portable surface scanner 
159 obtaining a 3D bone file (.obj format); similar files were output from the CT scan data (see

160 below) for Crocodylus. Meshlab software (Visual Computing Lab - ISTI - CNR, Pisa, Italy) was

161 used to reduce the resolution of the original .obj files as needed. Each individual bone file was

162 then imported to 3D Studio Max ${ }^{\circledR}$ software (Autodesk $^{\circledR}$, San Rafael, California, USA) in order to

163 articulate the shoulder girdle and forelimb and to define the degrees of freedom (DOF; i.e. the

164 possible axes of mobility) of each joint. We obtained our Crocodylus johnstoni specimen from

165 the St. Augustine Alligator Farm and Zoological Park (St. Augustine, Florida, USA), where it had

166 died of natural causes in captivity. This specimen was also used in studies by Allen, Paxton \&

167 Hutchinson (2009), Fujiwara \& Hutchinson (2012) and Allen et al. (2014), and was

168 approximately adult, with a total body mass of $20.19 \mathrm{~kg}$. It was scanned using a Picker PQ 5000

169 CT scanner (axial 512 x 512 pixel slices at 2.5mm thickness; 100mA, $120 \mathrm{kVp}$, resolution 1.024

170 pixels $\mathrm{mm}^{-1}$ ) and segmented in Mimics software (Materialise, Inc.; Leeuwen, Belgium) after CT

171 scanning for simple 3D modelling in the aforementioned studies, especially Fujiwara \&

172 Hutchinson (2012), who reconstructed the major forelimb muscles in a computational model

173 that we adapted here.

175 Joint axis estimation, reference pose and terminology

176 We first used the osteology of each bony joint to estimate the orientations of the 3D axes of

177 that joint (Fig. 2). Those axes also set up the translations required to place the bones in relation

178 to one another, from proximal to distal.

179 Considering that some extinct archosaurs have rather simply shaped appendicular

180 condylar areas, implying the presence of large amounts of epiphyseal cartilage (Fujiwara, Taru 
181 \& Suzuki, 2010; Holliday et al., 2010; Bonnan et al., 2013), thickness of soft tissues between the

182 joints needed to be accounted for. Consequently, we left $10 \%$ of the total forelimb length as

183 free space for epiphyseal cartilage in Mussaurus, distributed between the three main limb

184 joints (i.e. glenohumeral, elbow, wrist), following the estimates of Holliday et al. (2010).

Geometric objects were used to link adjacent segments, using spheres (gimbal/ball-andsocket) for the glenohumeral joints and cylinders (hinges) for the other joints. These objects established the centres of rotation of each joint, through which the axes of joint rotation were positioned. Next, we defined the rotational degrees of freedom (DOFs) that were allowed around each joint axis. Although translation is known to occur in extant archosaur forelimb joints (namely the glenohumeral joint [Baier \& Gatesy, 2013]), we judged the potential effects on moment arms to be relatively minor. For the glenohumeral and elbow joints, we inferred from the morphology that these joints might have three DOFs (extension/flexion, abduction/adduction, and pronation/supination) in both Crocodylus and Mussaurus. For Crocodylus, we also allowed three DOFs for the wrist joint, although only two DOFs for the wrist of Mussaurus, not allowing pronation/supination because the block-like configuration of the carpus (Galton \& Upchurch, 2004) and the fixed radius against the ulna (e.g., VanBuren \& Bonnan, 2012) probably precluded such motion. Finally, for the metacarpo-phalangeal, and interphalangeal joints (only for Mussaurus), we only allowed one DOF (extension/flexion), because the bony anatomy indicated that these joints acted almost exclusively as hinges. Our range of motion analysis (ROM, below) then considered how large the potential angular excursions of these DOFs might have been. 
In order to set the initial position of the models, a reference pose at which all joint angles

203

204

205

206

207

208

209

210

211

212

213

214

215

216

217

218

219

220

221

222

223

were set at $0^{\circ}$ was chosen (e.g. Hutchinson et al., 2005; Hutchinson et al., 2015; Baier \& Gatesy, 2013; Baier, Gatesy \& Dial, 2013). Thus the reference pose constituted a starting point from which comparisons could be made, facilitating understanding of what any value for a joint angle represents (vs. this reference angle, a fully straightened limb orientation, with the forelimb extended laterally, perpendicular to the vertebral column and body's craniocaudal axis) (Fig. 2A-F).

The segments of the forelimb were positioned following Baier \& Gatesy (2013), in which the humerus was laterally oriented, perpendicular to the vertebral column ( $0^{\circ}$ flexion) and its long axis was parallel to the ground $\left(0^{\circ}\right.$ abduction), whereas the axis connecting the medial and lateral distal condyles was parallel to the vertebral column and the deltopectoral crest pointed ventrally $\left(0^{\circ}\right.$ pronation). The major (longitudinal) axis of the ulna and radius (antebrachium) was parallel to that of the humerus $\left(0^{\circ}\right.$ extension/abduction), and again the mediolateral axes of the distal condyles were parallel to the vertebral column's longitudinal axis. Unlike the model of Baier \& Gatesy (2013), the curvature of the ulna was in a plane paralell to the long axis of the humerus ( $0^{\circ}$ pronation/supination). Finally, the manus was oriented with the long axis of the metacarpus parallel to the long axis of the antebrachium ( $0^{\circ}$ flexion and abduction), whereas the curvature of the ungual of the first manual digit was in the same plane as the long axis of the antebrachium ( $0^{\circ}$ pronation) (Fig. 2).

Rotations away from $0^{\circ}$ for each joint were defined as three successive rotations of the segment relative to the axis proximal to it (i.e. its reference position), in the order $x$ (e.g. pronation/supination), y (e.g. abduction/adduction) and z (e.g. extension/flexion). Our models 
224 had right-handed coordinate systems, so pronation (around $\mathrm{x}$ ), abduction (around y) and

225 extension (around $\mathrm{z}$ ) were negative values (i.e. of joint angle rotations), whereas supination,

226 adduction and flexion were positive values.

Finally, the articulated forelimb model in the reference pose was exported to musculoskeletal modelling software (Software for Interactive Musculoskeletal Modeling [SIMM]; Musculographics, Inc., Chicago, Illinois, USA) (Delp \& Loan 1995, 2000), using custom MATLAB code (The Mathworks, Inc., Natick, Massachusetts, USA).

There is not a general consensus regarding anatomical terminology among tetrapods because of their great morphological disparity (Harris, 2004; Wilson, 2006). Caution is thus warranted when attempting to compare animals with sprawling (ancestral, at some level for Archosauria) versus erect (derived) stances or postures (Gatesy, 1991; Padian, Li \& Pchelnikova, 2010) because each one can imply a different typical orientation for homologous or corresponding bones. To partly circumvent this problem, Jasinoski, Russell \& Currie (2006) used two terms for bone orientation: 'developmental' and 'functional' orientations. The term 'developmental orientation'refers to the ancestral (sprawling) state, which is equivalent to that often present in tetrapod embryos, especially forms with relatively plesiomorphic limbs (e.g. positions present in adults, which vary in different groups of tetrapods according to their locomotor mode(s) used. Our model oriented the forelimb segments from the most proximal to distal ones, starting with the scapula. We thus chose a developmental position of the scapula, which means that the scapular blade was initially oriented vertically and the glenohumeral joint was caudolaterally oriented, as retained by extant crocodiles. Positioning Mussaurus's scapula 
246 the same way as in Crocodylus ensured the same kind of movements (pronation/supination,

247 extension/flexion, abduction/adduction) around the same axes in both models, in the starting 248 configuration (i.e. reference pose).

249 As our reference ('developmental') pose did not necessarily reflect a biologically plausible pose (i.e. a pose that is mechanically allowed by their joints without risk of dislocation, or 'functional' orientation), a standardized, biologically plausible pose was also chosen in order to make realistic comparisons between taxa in terms of joint ranges of motion and moment arm analysis. Hence we used a 'resting' pose for both taxa, which was modified from the reference pose and represented an approximate in vivo plausible pose that was feasible for Crocodylus and (in our judgement based on the anatomy) Mussaurus. In our analysis, starting from the reference pose (all $0^{\circ}$ values), the resting pose for Mussaurus was set at $5^{\circ}$ of supination, $25^{\circ}$ of adduction and $-40^{\circ}$ of extension for the glenohumeral joint; whereas $70^{\circ}$ of flexion was chosen for the elbow. In Crocodylus (also starting from the reference pose), the same values were chosen as in Mussaurus, except for long-axis rotation (pronation/supination) at the glenohumeral joint, which remained at $0^{\circ}$ (Fig. 3). These admittedly were subjective judgements based on the joint morphology and function, but were deemed far more plausible than the reference pose and thus more suitable for biological comparisons. Hutchinson et al., 2005; Baier \& Gatesy, 2013), we used pronation and supination for long axis rotation, the former alluding to internal (medial) and the later to external (lateral) rotation. We expressed those DOFs relative to the axes of the reptilian saddle-shaped glenoid on which they 
268 also Baier \& Gatesy, 2013; Baier, Gatesy \& Dial, 2013). Hence the abduction/adduction axis lay

269 parallel to the long axis of the glenoid and the extension/flexion axis was perpendicular to the

270 long axis of the glenoid (Fig. 4A-C).

271 Whilst the reference pose was used as a common point of comparison in terms of the

272 DOFs, caution is warranted when one of the studied taxa (in this case Mussaurus) is shifted

273 from the reference pose (with a vertically oriented scapular blade and caudally-oriented

274 glenoid) to the resting pose (with a caudodorsally oriented scapular blade and a caudoventral

275 glenoid). Such reorientation of the glenoid (Jenkins, 1993; Gatesy \& Baier, 2005) entails drastic

276 modifications of the anatomical and functional implications of the joints' DOFs (except for

277 pronation/supination). This means that homologous movements in both poses are expressed as

278 different functions in each of them. Consequently, an abduction and adduction movement (i.e.

279 action) is expressed as elevation and depression (i.e. function) in the reference pose but

280 functions as retraction and protraction in the resting pose, respectively (Fig. 4D-G). This shifting

281 of joint functions must be kept in mind when comparing our results with those of previous work

282 (see Discussion). To minimize confusion and to keep consistency with the Crocodylus model, we

283 conserved the same terms for motions in the reference and the resting poses for Mussaurus,

284 rather than converting the resting pose's joint motions into different terms. That is, the

285 movement that describes abduction in the reference pose (i.e. the movement parallel to the

286 long axis of the glenoid), was also called abduction in the resting pose, with no reference to the

287 movement relative to the ground (i.e. elevation/depression; protraction/retraction) that the

288 limb would be performing, unless otherwise stated. 


\section{Muscle reconstruction}

291 Soft tissue inferences for the myology of Mussaurus patagonicus were established via reference

292 to the literature, by comparisons and homology hypotheses from previous studies of the

293 anatomy of living archosaurs as well as extinct forms (Cooper, 1981; Meers, 2003; Jasinoski,

294 Russell \& Currie, 2006; Langer, Franca \& Gabriel, 2007; Remes, 2008; Maidment \& Barrett,

295 2011; Burch, 2014; Allen et al., 2014; Klinkhamer et al., 2017) and via additional reference

296 dissections of two specimens of Caiman latirostris (Crocodylia, Alligatoridae) and Gallus gallus

297 (Aves, Galliformes). The Extant Phylogenetic Bracket (EPB, Witmer, 1995) was used to

298 formulate hypotheses about the soft tissue relations in extinct taxa that could be tested by

299 reference to the known osteological correlates of the soft tissues in fossil taxa enclosed by the

300 bracket, constraining speculation to a minimum (Witmer, 1995). We inferred the forelimb

301 muscles' origin and insertion sites for Mussaurus using this EPB method. Muscle nomenclature

302 used herein is based on Meers (2003) and Burch (2014). A total of 30 muscles were

303 reconstructed in Mussaurus (although some were summed into functional groups for some

304 actions). We avoided reconstructing muscles originating from the body wall (i.e. other than the

305 pectoral girdle or forelimb), including major shoulder muscles such as M. latissimus dorsi, M.

306 pectoralis, M. serratus and others including smaller muscles acting solely on the pectoral girdle

307 (e.g. M. rhomboideus, M. trapezius, M. levator scapulae). Plausible reconstructions of these

308 muscles in the future would require 3D geometry (ideally scanned skeletal material) of the

309 vertebrae, ribs and other elements as well as decisions about the ROMs of any joints therein.

310 M. pronator quadratus, a supinator of the radius-ulna, was not reconstructed in Mussaurus as

311 we did not infer motions within the antebrachium. Similarly, small distal carpus/manus muscles 
312 were omitted except where noted below. Muscle abbreviations and EPB levels of inferences are

313 given in Table 1. Our placements of the origin and insertion of each muscle qualitatively

314 approximated the centroids of the estimated areas of attachment inferred from crocodiles and

315 birds (following Hutchinson et al., 2005; Hutchinson et al., 2015). These centroid

316 approximations were used in the next step.

\section{Muscle path specification}

Once muscles were positioned at their respective origins and insertions, the next step was to model plausible paths over which each muscle would move during motion of the joints.

Otherwise a uniformly straight line of action of muscles would create unnatural paths, crossing over (or through) the bones or other muscles in implausible ways, resulting in dubious moment arms as outputs. We used 'via points' and 'wrapping surfaces' to create anatomically realistic paths. Via points are fixed points attached to a body segment that can be used to implement simple constraints on a muscle's path relative to a bone or other structure (Fig. S1). For example, the triceps muscle group, originating on the scapula/coracoid and the humeral shaft, needed via points to avoid the assumed shape that the more internally located muscles might have had, as exemplified by M. triceps brachii caput mediale 1 (TBM1) (internally placed) and M. triceps brachii caput scapulare (TBS) (externally placed). when crossed, preventing any associated muscle from penetrating it (Delp et al., 1990; see also 
333 Hutchinson et al., 2005; Hutchinson et al., 2015). Wrapping objects' attributes are listed in 334 Table S1.

335 Most wrapping objects were represented as cylinders, used to represent physical bone 336 surfaces, to constrain muscle paths, and to imitate unpreserved attributes (e.g. cartilage). This 337 latter point is very important because a large amount of articular cartilage is missing in extinct 338 reptiles, affecting the paths of muscles involved (Hutchinson et al., 2005). The elbow joint is

339 critical because our inferences of its morphology would be affected by missing articular cartilage (Fujiwara, 2010; Holliday et al., 2010) and the main elbow (and other distal) extensor and flexor musculature would pass closely around this joint, with their paths influenced by this cartilage. A set of cylinders, serving as wrapping surfaces for one or more muscles, was placed parallel to the humeral condylar axis at varied distances from the condyles (see sensitivity analysis of moment arms below and in Discussion), taking the role of the articular cartilage on constraining muscle paths around the elbow.

Considering that our Mussaurus model exhibited differences in joint orientations

between the reference and the resting pose, and the former was actually an implausible pose for a basal sauropodomorph, some muscle paths required additional constraints to fit the reference pose (Fig. S3; Table S1). Our complete models for the forelimbs of Crocodylus and

Mussaurus are available online (Otero et al., 2017a,b) at

https://figshare.com/s/2a11566a4cb9e57245ff and

\section{2 https://figshare.com/s/659a63ec758678866d9e.}


355 Analyses of the forelimb joints' ranges of motion (ROM) were conducted for both the reference

356 and resting poses, for each DOF allowed for each joint in the model. Estimation of ROM was

357 done with the musculoskeletal software, by manipulating each DOF manually and visualizing in

358 3D at which joint angles the bones came into close proximity and thus would pass through each

359 other (or their presumed cartilage) if moved further (e.g. Pierce et al., 2012; Reiss \& Mallison,

360 2014; Arnold et al., 2014). ROM estimation was not performed directly from bone to bone

361 surfaces, but rather left $10 \%$ space of total bone length distributed among the glenohumeral,

362 elbow and wrist joints for emulation of the cartilage volume that might have existed in life

363 (following Holliday et al., 2010). Thus our ROM analysis was roughly equivalent to 'ROM4' of

364 Hutson \& Hutson (2012) in which all soft tissues but cartilage was removed. Considering that

365 the reference pose was not a realistic posture, we expected that ROM values estimated from a

366 resting pose would be smaller because of the restrictions imposed by more realistic movements

367 of the joints.

368 The reference pose in this study represented how the joint was positioned at zero

369 degrees; thus, any angular rotation away from that pose would be either positive or negative.

370 To clarify further, when the models were positioned in the resting pose (or any other pose), the

$3710^{\circ}$ position was not altered, and any other positions of the limb segments were quantified

372 relative to that $0^{\circ}$ reference pose.

373

\section{Analysis of muscle moment arms}

375 We calculated muscle moment arms about the glenohumeral, elbow, and wrist joints for the

376 Mussaurus and Crocodylus models (in SIMM software, Delp et al., 1990; Delp \& Loan, 1995). 
377 Additionally, we explored muscle moment arms in extension/flexionfor manual digit I in

378 Mussaurus because this digit in early sauropodomorphs has a medially deflected claw that has

379 been hypothesized as having played a key role in manus functions other than locomotion, such

380 as grasping and browsing (Galton \& Upchurch, 2004; Yates et al., 2010).

For the glenohumeral joint, moment arms were calculated for all three rotational DOFs,

considering that movements of the humerus allowed for appreciable amounts of

extension/flexion, abduction/adduction, and pronation/supination in both Mussaurus and

Crocodylus (see ranges of motion below; additional plots are in the online Supplementary Text

as per the Results). For the remaining joints (i.e. elbow, wrist, metacarpo-phalangeal and

interphalangeal), only flexion and extension moment arms were calculated because this DOF

simplied our analysis (but see Discussion for the elbow joint).

Moment arms were first calculated in the Mussaurus and Crocodylus models for the

reference pose. If a muscle had a certain action for more than $75 \%$ of a given DOF's range of

motion, it was plotted as a 'pure' action muscle (e.g., 'pure' flexor), otherwise it was plotted as

'mixed'. If there was a mismatch between the taxa (e.g. a muscle being a flexor in Crocodylus

but an extensor in Mussaurus), then we also plotted that muscle in the 'mixed' action category.

We also calculated the moment arms for the Mussaurus model in the resting pose for comparison with the reference pose.

Moment arm values vary depending on the paths of muscles (Delp et al., 1990;

Hutchinson et al., 2005), so alteration of either origin or insertion sites (as well as paths 
399

400

401

402

403

404

405

406

407

408

409

410

411

412

413

414

415

416

417 RESULTS accordingly. Discussion.

actions. Here we focused our sensitivity analysis on the elbow of Mussaurus, for which the articular cartilage volume is unknown and likely was considerable (Schwarz, Wings \& Meyer, 2007). We started with a minimum amount of cartilage, increased the elbow cap to a maximum, and then evaluated how extensor muscle moment arms were affected by these assumptions (see Discussion). Here, the minimum and maximum amounts of cartilage were defined taking as a reference the standardized $10 \%$ of the total limb length previously proposed in the literature for archosaurs (Holliday et al., 2010) and then we added and removed cartilage

Our presentation of moment arm values required some normalization to facilitate comparisons between Crocodylus and Mussaurus, because these taxa differ so greatly in body size and forelimb morphology. Following typical practice (e.g. Hutchinson et al., 2008; Bates \& Schachner, 2012), we normalized the moment arms by corresponding segment lengths (humerus, antebrachium (radius-ulna) and metacarpal II for shoulder, elbow and distal joints; data in Table S2), using the distances between joint centres in the model as the lengths.

However, as forelimb proportions clearly changed between Archosauria and these two taxa, segment lengths are not the ideal metrics for normalization. We consider this problem in the

\section{Muscle reconstruction}

419 Non-avian archosaurs represent a particular challenge when reconstructing forelimb 
421 disparities, related to the different modes of locomotion existing between extinct and the living

422 forms (e.g., sprawling vs erect; biped vs quadruped; non-flying vs flying). Although the

423 inferences of presence/absence of the forelimb musculature reconstructed herein for

424 Mussaurus (Fig. 3) were based on the EPB approach (Witmer, 1995), our final decisions of

425 muscle position and extent (e.g. in equivocal cases; Level II' inferences) were based mainly on

426 extant Crocodylia because of the greater morphological similarities that this group shares with

427 non-avian dinosaurs than with birds (Jasinoski, Russell \& Currie, 2006; Remes, 2008; Burch, 428 2014; Maidment et al., 2014).

Within the shoulder musculature, one important difference from previous contributions

430 is the reconstruction of the M. teres major (TM) in Mussaurus. This muscle is absent in most

sauropsids (Remes, 2008) and was reconstructed neither in theropods (Nicholls \& Russell, 1985;

Jasinoski, Russell \& Currie, 2006; Burch, 2014) nor in basal ornithischians (Maidment \& Barrett,

2011). However, the TM is present in extant Crocodylia (e.g., Meers, 2003; Allen et al., 2014;

Klinkhamer et al., 2017), thus representing a Level II inference for an insertion on the humerus,

also inferred to have been present in the basal sauropodomorphs Saturnalia and Efraasia

437 (Remes, 2008).

The origin and insertion of $\mathrm{M}$. deltoides clavicularis (DC) are rather congruent among

different studies, taking origin from the acromial area of the scapula and inserting on the lateral

440 aspect of the deltopectoral crest of the humerus in both Crocodylia and non-avian Dinosauria

441 (Meers, 2003; Jasinoski, Russell \& Currie, 2006; Suzuki \& Hayashi, 2010; Maidment \& Barrett

442 2011; Burch, 2014; Klinkhamer et al., 2017). However, an origin from the clavicle was reported 
443 in lepidosaurs (Russell \& Bauer, 2008) and birds (Burch, 2014), but clavicles are only known for

444 the basal sauropodomorphs Massospondylus, Plateosaurus (Yates \& Vasconcelos, 2005) and

445 Adeopapposaurus (Martínez, 2009). Considering that there is no evidence of clavicles in

446 Mussaurus, such an origin site was not reconstructed here. Remes (2008), on the other hand,

447 proposed the presence of clavicles throughout sauropodomorph evolution, and that these were

448 the osteological correlate for the DC. Regardless, the origin site and the line of action of this

449 muscle would not be drastically affected by the presence of clavicles as reconstructed by Yates

450 \& Vasconcelos (2005) or Remes (2008).

451 The coracobrachialis (CB) muscle was reconstructed in Mussaurus with a single head (M.

452 coracobrachialis brevis), and two divisions of that (pars ventralis and dorsalis; CBV and CBD) as

453 in living crocodiles (Meers, 2003; Suzuki \& Hayashi, 2010; Allen et al., 2014; Klinkhamer et al.,

454 2017). There are two heads for this muscle (pars cranialis and caudalis) in extant birds, both

455 originating from the craniolateral aspect of the coracoid (Vanden Berge \& Zweers, 1993). Based

456 on its anatomical position, the M. coracobrachialis cranialis of birds should be equivalent to the

457 CBV of Crocodylia, and it would insert on the base of the deltopectoral crest of the humerus

458 (Vanden Berge, 1982). An additional head, M. coracobrachialis longus, was reported as absent

459 in Crocodylia (Jasinoski, Russell \& Currie, 2006; Remes, 2008; but see Nicholls \& Russell, 1985)

460 and present in some birds (Jasinoski, Russell \& Currie, 2006). Langer, Franca \& Gabriel (2007),

461 however, inferred the presence of this muscle in Saturnalia, taking into account that most

462 neognaths have it, although we do not agree with such an inference considering the drastic

463 modifications of avian forelimbs (i.e. a level II inference for Mussaurus) so we did not

464 reconstruct the M. coracobrachialis longus in Mussaurus. 
The supracoracoideus (SC) muscle has two heads in Alligator mississippiensis (Meers,

466

467

468

469

470

471

472

473

474

475

476

477

478

479

480

481

482

483

484

485

486

2003). In extant birds there is a single head, but with multiple origins (e.g. keel, mesosternum, manubrium, Vanden Berge \& Zweers, 1993; Jasinoski, Russell \& Currie, 2006). Homologies with Crocodylia are controversial, with no consensus on whether the scapular (Remes, 2008) or coracoid (Maidment \& Barrett, 2011) head was lost in birds. The origin site (either single or multiple) of the supracoracoideus complex is consistently located around the scapula-coracoid boundary in Crocodylia, and always inserts on the deltopectoral crest. Thus for the biomechanical purposes of this study, we reconstructed the SC in Mussaurus as a single head originating from the centroid of the area where any head(s) should have originated. In addition, that area is not preserved in any of the Patagonian specimens, precluding the identification of osteological correlates for the origin of this muscle.

The scapulohumeralis was reconstructed in Mussaurus as a single head ( $\mathrm{M}$.

scapulohumeralis posterior, SHP), corresponding to the M. scapulohumeralis caudalis of Crocodylia (Meers, 2003; Remes, 2008; Suzuki \& Hayashi, 2010; Allen et al., 2014; Klinkhamer et al., 2017). Scapulohumeralis anterior was not reconstructed in Mussaurus because it is absent in Crocodylia (Meers, 2003; Jasinoski, Russell \& Currie, 2006; Suzuki \& Hayashi, 2010; Burch, 2014), although it is reported in birds. The medial side of the scapula of Mussaurus has a long ridge extending parallel to both margins (ventromedial ridge, Otero \& Pol, 2013), which has been hypothesized as the boundary of SHP (ventrally) and SBS (dorsally) (Langer, Franca \& Gabriel, 2007; Burch, 2014).

The inferred number of heads of the Mm. triceps brachii (TB) for archosaurs are four (Jasinoski, Russell \& Currie, 2006; Burch, 2014) or five (Meers, 2003; Remes, 2008). In 
487 Mussaurus, as in extant Crocodylia we infer that there were two origin sites from the

488 scapulocoracoid (i.e. TBS, TBC) and two from the humeral shaft (i.e. TBL, TBM). Regardless of all

489 controversies surrounding the precise number of humeral heads in living archosaurs, for our

490 purposes of muscle moment arm analysis and considering the lines of action of this large

491 muscle group, the reconstruction of the humeral head in Mussaurus (TBM) was split into four

492 portions, which corresponded to the different areas on the humeral shaft from which the TBM

493 probably originated. Previous reconstructions of M. triceps in dinosaurs vary. The triceps group

494 was reconstructed with two scapulocoracoid heads and one humeral head in Saturnalia

495 (Langer, Franca \& Gabriel, 2007) and only two heads in early ornithischian dinosaurs (one from

496 the scapulocoracoid and one from the humerus; Maidment \& Barrett, 2011). Within later

497 ornithischians, five heads were inferred in Euoplocephalus (Coombs, 1978), corresponding to

498 those described for extant crocodiles.

499 The origin of the biceps brachii (BB) mucle in extinct forms is equivocal: some studies

500 place it just cranially to the glenoid lip of the coracoid (Langer, Franca \& Gabriel, 2007; Remes,

501 2008; Burch, 2014), whereas the origin in Crocodylia is even more cranially placed, close to the

502 scapulocoracoid boundary (Meers, 2003; Suzuki \& Hayashi, 2010), a hypothesis followed here

503 (see also Maidment \& Barrett, 2011). A second head of this muscle on the humerus (as present

504 in some birds, Remes, 2008) is too speculative (Level II') because it is absent in Crocodylia (e.g.

505 Meers, 2003) and no corresponding scars are evident in Mussaurus.

506 The brachialis (BR) and humeroradialis (HR) muscle attachments seem to retain their

507 ancestral origins and insertions in most sauropsids, with some secondary changes in birds. In

508 Mussaurus we infer that they originated from the humeral shaft, close to the deltopectoral 
509 crest, and inserted on the proximomedial surface of the radius (Jasinoski, Russell \& Currie,

510 2006; Remes, 2008; Burch, 2014). However, Cooper (1981), Langer, Franca \& Gabriel (2007) and

511 Maidment et al. (2011) placed the BR origin more distally, as in birds; a conclusion that we

512 deem to be less convincing (Level II' inference).

513 Most of the muscles originating from the humeral condyles and inserting either on the

514 radius or ulna, such as the supinator (SU), flexor ulnaris (FU) (anconeus sensu Burch, 2014), and

515 pronator teres (PT) do not exhibit major differences between extant Archosauria. Therefore,

516 qualitative reconstructions in extinct forms remain unequivocal (Remes, 2008; Burch, 2014; but

517 see Langer, Franca \& Gabriel, 2007), with the exception of the abductor radialis (AR), which is

518 not present in birds.

519 The M. extensor digitorum longus (EDL; extensor carpi ulnaris longus sensu Meers,

5202003 ) of sauropsids has an insertion that varies between the dorsal proximal portions of the

521 metacarpals, depending on the group (Remes, 2008; Burch, 2014). Insertions onto the bases of

522 metacarpals I and II are phylogenetically unequivocal for Mussaurus. Considering that

523 reconstructions of the insertion onto $\mathrm{MCl}, \mathrm{MCll}$ or both would not appreciably affect the EDL's

524 line of action as it crosses the wrist joint, we reconstructed this muscle as inserting only onto

525 MCII.

526

Remes (2008) inferred that sauropodomorphs lacked M. extensor carpi ulnaris (ECU),

527 but this is contradicted by evidence from crocodilian myology (dozens of specimens studied by

528 Meers, 2003; Allen et al., 2014) so our models incorporated the ECU for Crocodylus and

529 Mussaurus (also see discussion in Burch, 2014, 2017; Klinkhamer et al., 2017). The archosaurian

530 ECR (M. extensor carpi radialis longus sensu Meers, 2003) muscle inserts onto the radiale. 
531 Considering that basal sauropodomorphs lack a preserved radiale, we reconstructed the ECR as

532 inserting onto the distal carpus in Mussaurus (as in Aves). Similarly, the origin site of the

533 abductor pollucis longus (APL; M. extensor carpi radialis brevis sensu Meers, 2003) was placed

534 on the lateral side of the radius in Mussaurus. The osteological correlate of this origin was

535 assessed to be a small tubercle on the lateral and distal area of the radius, also reported in

536 Saturnalia (Langer, Franca \& Gabriel, 2007). An additional origin from the lateral ulna (Remes,

537 2008; Burch, 2014) was omitted because it would have had the same general line of action and

538 hence would not affect the action of this muscle in Mussaurus.

539 The flexor digitorum longus (FDL) muscle ancestrally had humeral, ulnar, and carpal

540 heads, all of them joining into a single tendon that then diverged to insert on the manual digits

541 (Meers, 2003). As one single common tendon passes across the wrist, we reconstructed only

542 the humeral head for Mussaurus, inserting on the flexor surfaces of the manual phalanges (i.e.

543 proximoventral aspect).

$544 \quad$ Nomenclature for the extensor and flexor musculature of the digits remains

545 controversial among living sauropsids, especially considering the extensive modifications of the

546 avian forelimb. Thus we withheld from reconstructing these muscles in detail for Mussaurus,

547 simply following the scheme from Meers (2003). Muscles extensores digitorum superficiales

548 (EDS) and profundus (EDP) in Mussaurus both originated from the distal aspect of metacarpal I

549 (for a similar myology, see Burch, 2014: extensores digitores breves, EDB), although leaving no

550 muscle scars. In extant birds, the EDP's putative equivalent by position would be the M.

551 extensor brevis alulae, but originating from the extensor apophysis of the metacarpus and the 
552 alula (Vanden Berge, 1982). Hence a Level II inference resulted for this muscle in Mussaurus,

553 and we applied the character state observed in Crocodylia rather than in Aves.

554 The flexores digitorum superficialis (FDS) and profundus (FDP) muscles originate

555 proximally from the distal carpals and distally insert onto the flexor process of the first phalanx

556 of the digits in Crocodylia (Meers, 2003; Burch, 2014). As with the extensor musculature of the

557 digits, avian homologues are difficult to establish, but judging from its position the M. flexor

558 alulae is a plausible candidate (Vanden Berge, 1982; Burch, 2014). We applied crocodylian

559 myology to Mussaurus.

560

561

Joint range of motion analysis

562 Here we consider the results of the range of motion (ROM) analysis for Mussaurus patagonicus

563 in the resting pose, whereas for Crocodylus johnstoni, ROMs for the reference pose were the

564 same as those for the resting pose (Tables 2, S3).

565 Pronation and supination values around the glenohumeral joint had similar values for

566 Mussaurus $\left(-25^{\circ} / 25^{\circ}\right)$ and Crocodylus $\left(-20^{\circ} / 20^{\circ}\right)$, for a total maximal ROM of $50^{\circ}$ and $40^{\circ}$,

567 respectively. The glenohumeral joint of Mussaurus allowed $-25^{\circ}$ of abduction and $40^{\circ}$ of

568 adduction for a total ROM of $65^{\circ}$. On the contrary, the Crocodylus model showed a reduced

569 capacity for abduction from the reference pose $\left(-5^{\circ}\right)$ and allowed $45^{\circ}$ of adduction, for a total

570 glenohumeral ROM of $50^{\circ}$. The flexion and extension axis of the glenohumeral joint in

571 Mussaurus allowed $-35^{\circ}$ of flexion and $-70^{\circ}$ of extension from the $0^{\circ}$ pose, for a total ROM of

$57235^{\circ}$. Crocodylus showed a greater glenohumeral ROM of $65^{\circ}$ ( $5^{\circ}$ flexion to $-60^{\circ}$ extension). 
Long axis rotation at the elbow showed interesting values in the Mussaurus model,

574 allowing $-30^{\circ}$ of pronation and $0^{\circ}$ of supination, for a total ROM of $30^{\circ}$. In Crocodylus, less

575 pronation than in Mussaurus was allowed $\left(-20^{\circ}\right)$ starting from the reference pose, but more

576 supination as well $\left(8^{\circ}\right)$, for a total of $28^{\circ}$ of long axis rotation. Abduction and adduction, on the

577 other hand, showed no differences between both Mussaurus and Crocodylus, allowing a total

578 ROM of $10^{\circ}\left(5^{\circ}\right.$ in each direction). Finally, flexion and extension at the elbow showed similar

579 ROM values in Mussaurus and Crocodylus, allowing flexion to $130^{\circ}$ in Mussaurus and $110^{\circ}$ in

580 Crocodylus. Extension values could not surpass $20^{\circ}$ in Mussaurus and $15^{\circ}$ in Crocodylus.

581 Pronation and supination at the wrist were precluded in Mussaurus. Abduction and

582 adduction showed the same values for Mussaurus $\left(-10^{\circ} / 10^{\circ}\right)$, whereas Crocodylus had more

583 abduction capacity $\left(-30^{\circ}\right)$ in contrast to adduction $\left(5^{\circ}\right)$. Flexion and extension of the wrist joint

584 was $70^{\circ}$ and $-30^{\circ}$, respectively for Mussaurus, whereas in Crocodylus flexion showed smaller

585 ROM values $\left(40^{\circ}\right)$ and extension ROM was greater $\left(-60^{\circ}\right)$ than in Mussaurus. The metacarpo-

586 phalangeal joint of digit I in Mussaurus had $50^{\circ}$ of flexion and $-40^{\circ}$ of extension ROM, whereas

587 the interphalangeal joint allowed the angle of flexion to increase to $70^{\circ}$ and extension could 588 reach $-25^{\circ}$.

\section{Muscle moment arm analysis}

591 Here we compare the moment arm values obtained for Crocodylus and Mussaurus in the

592 resting pose. For the glenohumeral joint, moment arms were measured while varying the

593 degree of freedom they relate to (pronation/supination, extension/flexion, and

594 abduction/adduction) to quantify how joint orientation affected muscle action. We also plotted 
595 glenohumeral pronation/supination and abduction/adduction moment arms against

596 glenohumeral extension/flexion angle (see online Supplementary Text). For the elbow and

597 wrist, we focused on extension/flexion moment arms and joint angles because this is the main

598 motion performed by those joints. Then we focus on inferences about broad trends in muscle

599 actions (and, where feasible, general functions) inferred from the resting pose.

600

601 Glenohumeral joint (Figs. 5-7, S8,S9; Tables 3, S4)

602 Some muscles showed similar actions for pronation and supination around the glenohumeral

603 joint in Crocodylus and Mussaurus, whereas others displayed differences in muscle action

604 between taxa (Fig. 5). Muscles originating from the scapular blade and inserting well lateral or

605 well medial on the proximal humerus had the same action for both taxa; e.g. some humeral

606 supinators such as DC, DS and TM. TBC and SC consistently were supinators, although the TBC

607 inserted on the olecranon process of the ulna rather than on the humerus in both taxa, and the

608 SC originated on the proximal scapula and coracoid and not on the scapular blade. The SBS and

609 CBV remained as humeral pronators for both taxa (Fig. 5A).

610 In contrast, BB, SHP, TBS and CBD had variable actions that differed between Mussaurus

611 and Crocodylus (Fig. 5C). SHP acted as a supinator in Crocodylus, but was a pronator in

612 Mussaurus; BB and TBS were fully a supinators in Crocodylus, but had a mixed action in

613 Mussaurus. Finally, CBD had very different actions in the two taxa: in Crocodylus it switched

614 between weak pronation/supination whereas in Mussaurus it was consistently a strong

615 supinator. 
617 in Crocodylus and Mussaurus for moment arm magnitudes around the glenohumeral joint, 618 especially as joint orientation was varied between supination and pronation (Fig. 5B,C). For example, most supinator muscles in Crocodylus (DC, SHP, TM, SC, TBS, TBC and BB) experienced an increase of their moment arms with pronation. In contrast, in Mussaurus, only two (SC and TBC) of the six supinator muscles increased their supinator moment arms with pronation. The

622 remaining glenohumeral supinators in Mussaurus (DS, DC, TM and CBD) displayed patterns of 623 increasing supinator moment arms with glenohumeral supination, not pronation. Pronator 624 muscles (e.g. CBV), however, displayed similar patterns in both taxa (Fig. 5A), showing a general 625 increase of pronator moment arms with supination; with the exception of the SBS muscle in 626 Mussaurus, which exhibited almost constant large moment arms. SBS, SHP, TBC and TM were extensors in both taxa (Fig. 6A), as well as TBS in Crocodylus. 628 On the other hand, the BB, CBD, SC, DS and DC muscles, which originated from the cranial 629 surface of the scapulocoracoid, were shoulder flexors in both taxa (Fig. 6B). mainly a weak shoulder extensor in Crocodylus (shifting from extension to flexion at about $-15^{\circ}$ ) but it had a strong, consistent flexor action action in Mussaurus (Fig. 6C). TBS, in contrast, consistently had a strong shoulder extensor action in Crocodylus but was a mainly a weak flexor in Mussaurus (shifting to extensor action at about $-65^{\circ}$ ).

636 flexion in both taxa was SBS. The remaining extensor muscles exhibited different patterns (Fig. 637 6A). In Crocodylus, all flexor muscles increased their moment arm about the glenohumeral joint 
638 with flexion, except for the CBD, which displayed less change (Fig. 6B). In Mussaurus, DS, DC

639 and CBV also increased their flexor moment arms with joint flexion, whereas SC and BB showed

640 an increase of flexor moment arms with joint extension.

641 We found that muscles acting about the glenohumeral abductor/adductor axis displayed

642 similar patterns in Crocodylus and Mussaurus. Many muscles (DC, DS, SHP, TBC, TBS and TM)

643 were glenohumeral abductors in both Crocodylus and Mussaurus (Fig. 7A). The BB, CBV, SBS

644 and SC muscles, however, had adduction actions for both taxa (Fig. 7B), whereas CBD acted as

645 an adductor in Crocodylus and as a mixed abductor/adductor in Mussaurus (Fig. 7C).

646 The glenohumeral abductor muscles DC, DS and TBS increased their moment arm with

647 joint abduction in Crocodylus, whereas all abductor muscles (DC, DS, SHP, TBC, TBS and TM) in

648 Mussaurus showed the same pattern (Fig.7A). In Crocodylus, most adductor muscles (CBD, CBV,

649 BB and SC) increased their moment arms with glenohumeral joint adduction, except for SBS and

650 CBD, which had the opposite pattern (Fig. 7B,C). In Mussaurus, BB and SC increased their

651 adduction moment arms with humeral abduction, whereas SBS and CBV remained rather

652 constant and only CBD increased its adduction moment arm with joint adduction (becoming an

653 adductor as the shoulder was adducted past $-10^{\circ}$; Fig. 7C).

654 While the above actions generally held across their same DOFs (e.g.

655 pronation/supination moment arms vs. joint angles), there were some interesting variations

656 when other DOFs were plotted. Pronator/supinator actions varied with glenohumeral

657 extension/flexion angle (Fig. S8), showing that while the SBS and CBV retained the same actions

658 across this DOF, other muscles such as the SC group became more mixed in their actions

659 especially in Mussaurus - and despite its limited glenohumeral ROM around that axis. TBC and 
660 BB even switched entirely to pronators in Mussaurus, and TBS became more mixed action than

661 just a supinator in Crocodylus. Moreover, abductor/adductor actions shifted slightly when 662 plotted the same way-most noticeably, the CBD and SBS muscles switched positions (cf. Fig.

663 7B,C vs. Fig. S9B,C), with Mussaurus and Crocodylus both having consistent adductor actions for 664 the CBD whereras the SBS stayed solely an adductor in Mussaurus but took on a more mixed 665 action in Crocodylus.

666

667 Elbow joint (Fig. 8; Tables 3, S5)

668 Although elbow adduction and abduction occur during 'sprawling' locomotion in Alligator 669 (Fujiwara \& Hutchinson, 2012; Baier \& Gatesy, 2013) and we allowed three DOFs at this joint in 670 both models, here we only consider flexion and extension for the purpose of our moment arm 671 analyses (but see below), because we expect elbow extension/flexion to have predominated in 672 Mussaurus. Generally, muscle actions around the elbow showed fewer differences between 673 Crocodylus and Mussaurus compared to the glenohumeral joint. In addition, the major elbow 674 extensors and flexors had similar patterns in both taxa, although varying in their relative 675 moment arm magnitudes.

676 The triceps group includes the main elbow extensor muscles. In Crocodylus and 677 Mussaurus, these muscles maintained a similar pattern of action (Fig. 8A), with smaller values 678 at full extension, mostly increasing their extensor moment arms as the elbow was flexed. The 679 most noteworthy difference between the action of the Triceps group in both taxa was that the 680 moment arm value in Mussaurus substantially increased (almost twice as large) between full 681 extension to full flexion, whereas in Crocodylus the values at maximal flexion and extension did 
682 not differ greatly. In both taxa, the peak values of moment arms occurred neither at full

683 extension nor full flexion, but at moderate elbow joint angles ( $\left.60-100^{\circ}\right)$.

684 Elbow flexor muscles revealed similar patterns in Crocodylus and Mussaurus, generally

685 increasing their flexor moment arm with increasing joint flexion (Fig. 8B). The BB, BR and HR all

686 reached peak flexor moment arms (about two times the minimal values) at moderate elbow

687 flexion angles $\left(\sim 90^{\circ}\right)$. In contrast, the PT and FDL only acted as weak elbow flexors in

688 Mussaurus, with minimal changes of their moment arms.

689 The remaining muscles acting around the elbow joint corresponded to those originating

690 on the distal humeral condyles. We found that, in Mussaurus, most of these antebrachial

691 muscles (AR, SU, ECR, ECU, EDL and FU) shifted from flexor to extensor moment arms as the

692 elbow became more flexed (between $55^{\circ}-65^{\circ}$; Fig. 8C). One interesting difference observed

693 between Crocodylus and Mussaurus was that, apart from the triceps group, different muscles

694 acted as elbow extensors in the crocodile (AR, SU, ECR, EDL, PT and FU); FDL being the only

695 mixed-action elbow muscle in Crocodylus (but a consistent flexor in Mussaurus). The FU muscle

696 in Crocodylus had the largest moment arm and remained an extensor throughout its ROM;

697 unlike in Mussaurus. Likewise, the PT muscle was always an elbow extensor in Crocodylus but a

698 flexor in Mussaurus. Overall, more muscles acting around the elbow of Mussaurus tended to

699 act as flexors, compared to the pattern in Crocodylus.

700

701

702 Wrist and manus joints (Figs. 9-11; Tables 4, S6, S7) 
703 Muscles acting around the distal forelimb joints in Crocodylus and Mussaurus showed minimal

704 switches of action; only in some cases switching at extremes of extension/flexion ROM (Figs. 9-

705 11). General patterns of moment arm changes with joint angles did not differ remarkably,

706 either. In both taxa, there were multiple carpal extensors including the ECR, ECU, APL and EDL

707 (Fig. 9A). Most of these muscles increase their moment arm with joint flexion (except for the

708 EDL in Crocodylus, which maintained almost constant extreme values). Similarly, the FDL was a

709 carpal flexor in both taxa, increasing flexor moment arm with joint extension (Fig. 9B). Moment arms for manus digit I were analyzed only for Mussaurus. Regarding muscles crossing the metacarpo-phalangeal joint ('MCP' in Fig. 10), the EDP and EDS exhibited similar patterns (due to their common paths) of reduced extensor moment arms with MCP joint extension (Fig. 10A), whereas the FDL, FDPI and FDSI showed a peak (flexor) moment arm at a moderate joint angle (Fig. 10B). EDP and EDS switched from flexor to extensor moment arms at about $-35^{\circ}$ of extension, increasing their moment arms until they reached an approximate plateau near $0^{\circ}$ (Fig. 10A), whereas FDL (and FDP, FDS) acted fully as a flexor (showing a pattern very similar to that for the metacarpo-phalangeal joint; Fig. 11B vs. Fig. 10B). For the interphalangeal joint ('INP' in Fig. 11), EDP and EDS showed similar patterns, being extensor muscles that increase extensor moment arm with joint extension, whereas FDL is a fully flexor, also increasing its flexot moment with joint extension.

721

\section{DISCUSSION}

723 Here, first we compare the results of our forelimb joint ROM analysis in Mussaurus and 724 Crocodylus considering these in light of conclusions from previous studies of this topic in other 
725

726

727

728

729

730

731

732

733

734

735

736

737

738

739

740

741

742

743

744

745

746

sauropodomorphs and theropods. Second, we compare the patterns of muscle moment arms in our two study taxa in the context of the evolution of muscle function across Archosauria, comparing with previous qualitative studies dealing with archosaur forelimb myology in which muscle function has been inferred (e.g. Meers, 2003; Jasinoski, Russell \& Currie, 2006; Langer, Franca \& Gabriel, 2007; Allen et al., 2014; Burch, 2014; Klinkhamer et al., 2017). As an important component of this comparison of muscle moment arms, we discuss the effects of altering: (1) joint posture (e.g. reference vs. resting pose); and (2) articular cartilage extent around the elbow in Mussaurus. Finally, we review the evolution of manus pronation in Sauropodomorpha in the light of our results for joint ROM and, where potentially relevant, muscle moment arms.

\section{Joint ROM analysis: Implications for the evolution of forelimb posture in sauropodomorphs}

Our analysis considered how forelimb joint ROM in Mussaurus differed between the reference and resting poses as well as how the estimated ROM compared with Crocodylus and various saurischian dinosaurs (as previous studies estimated). Here we also evaluate how our findings might reflect potential evolutionary trajectories of maximal forelimb joint ROM in Archosauria, as well as the limitations of these ROM assessments and comparisons. Where relevant, in tandem we also consider our results for muscle moment arms.

The most conservative DOF around the glenohumeral joint in Crocodylus and Mussaurus was pronation and supination (Tables 2, S3), showing grossly similar values in Crocodylus and Mussaurus. This similarity can partly be attributed to the relatively conserved morphology of the glenohumeral joint surfaces in both taxa, in which the scapular and coracoid lips form an 
747 inverted ' $V$ ' surface. Additionally, the potential ROMs in pronation and supination were

748 relatively independent of the orientation of the glenoid (or pectoral girdle and forelimb) and

749 hence, the same values were estimated for the reference and resting poses of Mussaurus (-

$75025^{\circ} / 25^{\circ}$ in pronation/supination), with almost the same values found for Crocodylus johnstoni (-

$75120^{\circ} / 20^{\circ}$ ). It is reassuring that the latter values were crudely similar to the actual ROM used in

752 vivo during walking in Alligator mississippiensis $\left(-17.9^{\circ} / 27.2^{\circ}\right.$ pronation/supination, Baier \&

753 Gatesy, 2013). However, our ROM results are smaller than those obtained by Pierce, Clack \&

754 Hutchinson $\left(2012 ;-75^{\circ} / 70^{\circ}\right.$, pronation/supination) for fleshed specimens of $C$. niloticus as well

755 as results (approximated as 3D) for A. mississippiensis (Hutson \& Hutson, 2013). However,

756 skeletonized specimens (e.g. the crocodile used in the present study) might underestimate

757 ROMs vs. fleshed ones (Hutson \& Hutson, 2012, 2013), although this is controversial (Pierce,

758 Clack \& Hutchinson, 2012; Arnold, Fischer \& Nyakatura, 2014; Kambic, Roberts \& Gatesy, 2017),

759 probably depending strongly on methods and investigators as well as definitions of 3D joint

760 axes and DOFs. Thus any corroboration of our ROM results for pronation/supination in

761 Crocodylus remains tentative.

762 In contrast, the remaining glenohumeral DOFs (extension/flexion and

763 abduction/adduction) exhibited different ROM values in both taxa but also in the reference and

764 the resting poses for Mussaurus, which are linked directly to the orientation of the

765 glenohumeral joint. Interestingly, in both the reference and resting poses, Mussaurus displayed

766 a larger ROM for glenohumeral abduction than Crocodylus; whereas Crocodylus had greater

767 capacity for adduction but only if starting in the reference pose (Tables 2, S3). This difference

768 was probably because of the smooth, broader glenohumeral surface in Mussaurus. It remains 
769 problematic that the extent and shape of glenohumeral articular cartilage in Mussaurus is

770 unknown, and we used admittedly simple models of the joint, but our models are provided with

771 this study so that others might build upon our efforts.

772 A major focus in studies of basal sauropodomorph locomotion is the likelihood of habitual

773 quadrupedalism (Jaekel, 1910; Fraas, 1913; Galton, 1990; Bonnan \& Senter, 2007, Bonnan \&

774 Yates 2007; Mallison, 2010a; Mallison, 2010b; Yates et al., 2010; VanBuren \& Bonnan, 2012;

775 Hutson, 2015). However, the ROMs of the forelimb joints depend on the morphology of the

776 articular surfaces (e.g. a wider glenoid surface should allow larger ROMs), and the orientation

777 of such articular surfaces will ultimately determine the way in which ROMs will influence

778 forelimb function (e.g. Jenkins, 1993; Gatesy \& Baier, 2005). Our ROM analysis of Mussaurus

779 indicated that if the scapula were oriented in an anatomical position of about $55^{\circ}-60^{\circ}$ from the

780 horizontal (i.e. caudodorsally), the maximal humeral protraction (i.e. glenohumeral joint

781 abduction) allowed would not pass vertical, which concurs with previous reports for other basal

782 sauropodomorphs (Bonnan \& Senter 2007; Mallison, 2010a; Mallison, 2010b) and theropods

783 (Senter \& Robins, 2005). This inference partially contradicts the possibility of quadrupedalism

784 as a habitual mode of locomotion in early sauropodomorphs such as Mussaurus. However, if

785 the elbow was habitually flexed during locomotion, then quadrupedalism might be more

786 achievable, perhaps with shorter stride lengths (Maidment \& Barrett, 2012). However, it

787 remains questionable how flexed the limbs of such a large animal as an adult Mussaurus would

788 have been (e.g. Biewener, 1989). Our inference contrasts with the condition inferred in

789 sauropods, in which the glenoid was more ventrally oriented but a caudodorsal scapular blade

790 orientation was maintained (Schwarz, Frey \& Meyer, 2007). A ventrally oriented glenoid seems 
791 to have allowed sauropods to protract their humerus cranial to vertical, facilitating

792 glenohumeral abduction (i.e. joint movement parallel to the long axis of the glenoid) during 793 quadrupedal locomotion.

794 Additionally, for the glenohumeral extension/flexion axis, Mussaurus exhibited a combination of peak moment arms at full extension (BB, CBD, SC, TBC, TM and SHP) but also at full flexion (DC, DS, SBS, CBV and TBS), although Crocodylus displayed mostly peak moment arms at full flexion (Fig. 6). In the case of the glenohumeral abduction/adduction axis, Mussaurus had peak moment arms with a more abducted humerus than in Crocodylus (Fig. 7).

These results indicated that Mussaurus had greater leverage with a more abducted

800 glenohumeral joint (i.e. humerus) than in Crocodylus, but the consequences of this leverage, and of glenohumeral posture in extension/flexion, are ambiguous although they hint at important functional differences in the forelimb muscles of these two taxa. both Crocodylus and Mussaurus were at full extension (i.e. a more columnar forelimb). Nonetheless, it is interesting to note that peak extensor moment arms were reached at different elbow joint angles in both taxa. In Crocodylus, peak moment arms were at joint angles of $\sim 45^{\circ}-55^{\circ}$ (Fig. $8 \mathrm{~A}$ ), which implies that leverage could be maximized at a moderately flexed elbow joint. In Mussaurus, elbow extensor moment arms at maximal extension in the resting pose fell drastically to minimal values. Regardless, peak moment arms around the elbow were

810 present at about $90^{\circ}$ (Fig. 8A), meaning that maximal muscle leverage was achieved at an even

811 more flexed elbow than in Crocodylus. This mechanical benefit of increased elbow flexion in 812 Mussaurus could be speculated to argue against a forelimb with strong specialization for 
813 supportive or locomotor functions (e.g. Biewener, 1989), and thus inconsistent with habitual

814 quadrupedalism in Mussaurus.

815 Overall, Crocodylus and Mussaurus showed interesting postural dependencies of their

816 muscles' moment arms, but the consequences for quadrupedalism in either taxon are unclear.

817 Likewise, divergent results for moment arm analyses in the hindlimbs of Tyrannosaurus (peak

818 extensor moment arms near full joint extension; Hutchinson et al., 2005) and ostriches

819 (extensor moment arms seeming to be suboptimal for antigravity support in walking and

820 running; Hutchinson et al., 2015) make it difficult, at present, to use these data to test

821 inferences about habitual joint posture. Regardless, all of these studies' findings reveal how

822 sensitive the moment arms of muscles are to joint orientation. Hence, assuming a constant

823 moment arm is far more risky than checking for this sensitivity.

824 Similar to pronation and supination around the glenohumeral joint, we found the ROM of

825 flexion and extension around the elbow to be rather conservative between Crocodylus and

826 Mussaurus, even though epiphyseal cartilage in the early sauropodomorph cannot be assessed

827 with great confidence. Full elbow extension $\left(0^{\circ}\right)$ was only allowed (indeed, required) in the

828 reference pose (Table S3), whereas maximal extension in the resting pose was $15-20^{\circ}$ for both

829 taxa, avoiding full extension of the elbow. These limits on elbow extension are similar to those

830 found for the basal tetrapod Ichthyostega (Pierce, Clack \& Hutchinson, 2012), the crocodylian

831 Alligator (Hutson \& Hutson, 2012; Baier \& Gatesy, 2013), basal saurischians (Sereno, 1993),

832 quadrupedal ornithischians (Maidment \& Barrett, 2012), basal sauropodomorphs (Bonnan \&

833 Senter, 2007; Mallison, 2010b; Vargas-Peixoto, Da Rosa \& Franca, 2015), non-avian theropods

834 (Senter \& Robins, 2005; White et al., 2015) and birds (Baier, Gatesy \& Dial, 2013). Thus our 
835 models reject the inference that Mussaurus would have routinely used a fully columnar

836 forelimb pose. This inference also supports the conclusion that no matter if manipulation is

837 being done with fleshed (Hutson \& Hutson, 2012) or skeletonized material (Sereno, 1993;

838 Senter \& Robins, 2005; Bonnan \& Senter, 2007; Mallison, 2010b; Pierce, Clack \& Hutchinson,

839 2012; Vargas-Peixoto, Da Rosa \& Franca, 2015; White et al., 2015), elbow hyperextension close

840 to $180^{\circ}$ leads to a high risk of disarticulation. Similarly, maximal elbow flexion was $110-130^{\circ}$ in

841 the two taxa (regardless of resting or reference pose), so the total ROM was less in the resting

842 pose vs. the reference pose.

843 Although we focused on extension/flexion as the major DOF considered for our analysis

844 of elbow and wrist biomechanics, it may be that Mussaurus (like Crocodylus) was capable of

845 other elbow and wrist motions, to some smaller degree. We found some elbow ROM in

846 pronation/supination $\left(\sim 30^{\circ}\right)$ and abduction/adduction $\left(\sim 10^{\circ}\right)$ for both taxa (Tables 2, S3). These

847 findings stand in contrast to some other studies which have inferred negligible ROM for these

848 joints in sauropodomorphs, archosaurs or tetrapods more generally (Senter, 2005; Senter \&

849 Robins, 2005; Bonnan \& Senter, 2007; Hutson \& Hutson, 2012; White et al., 2015). However,

850 the best representations of such joint motions we are aware of are three-dimensional, in vivo

851 quantitative measurements in taxa such as Alligator (Baier \& Gatesy, 2013), which found, for

852 example, detectable ROMs of $20-30^{\circ}$ for elbow pronation/supination and abduction/adduction

853 during walking. Roughly similar magnitudes of in vivo elbow motion were recently reported for

854 skinks by Nyakatura et al. (2014: their table 2).

855 Thus there is need for more integration of precise in vivo studies of actual joint motions

856 used by functioning animals (see also Ren et al., 2008: their table 3; Arnold, Fischer \& 
857 Nyakatura, 2014; Kambic, Roberts \& Gatesy, 2017), estimates of maximal ROMs from

858 morphology and other evidence such as fossil trackways (e.g. Norman, 1980, 1986; Carrano \&

859 Biewener, 1999; Blob \& Biewener, 2001; Bates et al., 2008; Arnold, Fischer \& Nyakatura, 2014).

860 How much of their arthrologically-apparent joint ROMs do real animals use in different

861 behaviours and do they ever naturally use motions that might seem to be 'dislocations' based

862 upon osteology or even whole-cadaver studies (Kambic, Roberts \& Gatesy, 2017)? How can

863 such data (including improved reconstructions of articular cartilage; see section below) enhance

864 estimates of joint ROM and locomotor function/evolution in extinct tetrapods? At present, we

865 are not convinced that the forelimb joints distal to the glenohumeral joint in archosaurs such as

866 Mussaurus were restricted to purely extension/flexion movements or that other motions were

867 negligible. Yet this controversy is more likely one of a matter of degree, not binary

868 presence/absence of non-parasagittal motions. There is apparently no remaining controversy

869 that extension/flexion motions of the limb joints of dinosaurs and many other archosaurs were

870 the largest ROMs used in vivo or allowed by the joints; our results continue to uphold that

871 inference (Tables 2, S3).

872 However, wrist osteology in Mussaurus makes reconstruction of abduction/adduction and

873 pronation/supination ROM difficult to do with much confidence. Indeed, understanding of the

874 mobility of the wrist joints among early sauropodomorphs is limited because there is a lack of

875 information regarding the osteology of the proximal carpus, and the distal carpus is

876 represented by two or three elements with a 'block' configuration (Senter \& Robins, 2005;

877 Bonnan \& Senter, 2007; Mallison, 2010a; Mallison, 2010b). The only wrist DOF inferred with

878 confidence in Mussaurus is flexion and extension, although even this is speculative. In the case 
879 of Crocodylus, the presence of proximal carpal elements allows more confident interpretations

880 regarding pronation/supination and abduction/adduction (e.g. Hutson \& Hutson, 2014),

881 exhibiting greater ROMs than estimated for Mussaurus (Table 2). Nonetheless, in addition to

882 the issues of cartilage non-preservation noted above, considerable pronation and supination

883 movements have been reported for the hindlimb bones of Alligator mississippiensis during

884 walking (Gatesy, 1991; Blob \& Biewener, 2001).

885 Within digit I in Mussaurus, both the metacarpo-phalangeal and the interphalangeal joints

886 present in early sauropodomorphs (and in dinosaurs in general) displayed ginglymoid, well-

887 defined articular surfaces, contrasting with the flatter (and sometimes pitted) ends of their

888 proximal long bones, for which thick articular cartilage has been inferred (Schwarz, Frey \&

889 Meyer, 2007; Holliday et al., 2010; Bonnan et al., 2013). In Mussaurus, we inferred that minimal

890 hyperextension ROM ( -25 to $\left.-40^{\circ}\right)$ was possible for both joints, with flexion predominating (50

891 to $70^{\circ}$ maximum), which would be similar to the condition reported for the ungual of digit I of

892 Massospondylus (Cooper, 1981) and the digits of Plateosaurus (Reiss \& Mallison, 2014; also

893 White et al., 2015 for the theropod Australovenator). Despite that basal sauropodomorphs

894 share similar manus morphology, more work is needed to test if there are any detectable

895 differences in ROM within this lineage. A limited amount of phalangeal hyperextension has

896 been proposed to be evidence against quadrupedalism because it might also limit the stride

897 length of the forelimb, particularly for the short forelimbs of early sauropodomorphs (Reiss \&

898 Mallison, 2014). However, to the degree that any such limitation imposed on stride length

899 existed, it would have been modest relative to the influences of the ROMs of more proximal

900 joints, considering their associated segments' greater lengths and thus the arcs swept for a 
901 given amount of joint ROM. Furthermore, ROMs of the same joints in quadrupedal

902 sauropod(omorph)s still deserve careful study for comparison, as it is questionable whether

903 phalangeal joint motion was important early in the evolution of their quadrupedalism, given the

904 rapid appearance of a columnar, bundled manus in sauropods (Bonnan, 2003; Bonnan \& Yates,

905 2007). Regardless, the mobility of digit I in the manus would be important for other non-

906 locomotor behaviours such as grasping and thus deserves study in more taxa and ultimately in a

907 phylogenetic context.

908

909

Archosaur forelimb muscle actions: Major differences between sprawling quadrupedalism

910

and erect bipedalism

911

Although the hindlimbs are/were a terrestrial locomotor module in essentially all archosaurs

912 (living and extinct), the biological role of the forelimbs varies, depending on the locomotor

913 pattern of the organism. Facultative bipedal vertebrates tend to devote the forelimbs to

914 biological roles other than solely body support or locomotion; e.g. manipulation, digging,

915 display and combat. Consequently, among our most interesting findings are estimates of how

916 the mechanical actions of some (but not all) muscles appear to differ between the more

917 sprawling forelimb posture of Crocodylus; presumably at least somewhat similar to the

918 ancestral locomotor pattern of basal archosaurs; to the more derived, erect, at least

919 facultatively bipedal pattern in Mussaurus. More studies are certainly needed to test how much

920 our assumption that Crocodylus's joint ROM and moment arm patterns are similar to those of

921 ancestral archosaurs (but see Parrish, 1986) and if Mussaurus's patterns are typical for

922 Sauropodomorpha, especially close to the origin of Sauropoda, but our estimates are important 
923 first steps in this direction. Although quantitative functional shifts have been proposed

924 previously for hindlimb muscles in various archosaurs (e.g. Hutchinson et al., 2008; Hutchinson

925 et al., 2015; Bates \& Schachner, 2012; Maidment \& Barrett, 2012), quantitative data for such

926 shifts in the forelimbs of extinct archosaurs have not been reported before.

927 It is important to note that our study considers muscle actions around the three main

928 DOFs (i.e. pronation/supination, extension/flexion, abduction/adduction) for the glenohumeral

929 joint, which had substantial mobility in both taxa modelled. Past studies, however, have tended

930 to focus on major muscle actions around a single axis, sometimes implicitly assuming that

931 actions around other axes were negligible or unimportant, but more often simplifying muscles

932 to only have one major action (see also Hutchinson et al., 2015; Rankin, Rubenson \&

933 Hutchinson, 2016 for similar points regarding 3D actions and broader biomechanical functions -

934 e.g. strut, motor, spring, brake, damper - in the pelvic limb muscles of ostriches). An advantage

935 of our musculoskeletal modelling approach is that, once constructed, actions in any directions

936 can be quantified, and these models could be used in the future to test broader issues about

937 biomechanical functions, biological roles and (with the addition of more musculoskeletal

938 models) comparative evolutionary patterns.

939 In the above context, the first part of the following section focuses on the influence of

940 the reference versus resting pose on muscle function, exploring how muscles respond to the

941 shifting from ancestral to derived limb postures in our studied taxa. We then examine how

942 moment arms in the resting pose differ between Crocodylus and Mussaurus, in all cases trying

943 to identify the muscles with actions most influenced by morphology and/or posture. Finally, we 
944 reflect on our findings in light of the challenge presented in finding an 'ideal' metric by which to

945 normalize moment arm values for comparisons between taxa.

946

947 Influence of the reference versus resting pose on muscle actions

948 When we compared the muscle actions estimated for the reference and resting poses for our

949 two study taxa, noteworthy differences appeared for the glenohumeral joint, whereas the

950 elbow and wrist joints did not exhibit pronounced differences between the poses (Tables 4, S5-

951 S8). This is discussed more in the Supplementary Text. Most muscle groups crossing the

952 glenohumeral joint that we analysed in the reference pose had evidence for differences of

953 action between Crocodylus and Mussaurus (7 out of 13 of the muscles in at least one DOF).

954 Furthermore, 8 out of 18 muscles acting around the elbow had differences of extensor/flexor

955 action between the two taxa, but no muscle crossing the wrist displayed differences of action

956 (Figs. 9, S7; Tables, 4, S8). In the case of the resting pose, differences in muscle action between

957 Crocodylus and Mussaurus were slightly less marked for the shoulder, with 5 out of 13 of the

958 muscles analysed having differences of action in at least one degree of freedom (Tables 3, S8).

959 Considering that both musculoskeletal models were set in the same reference pose, in

960 equally sprawled limb orientations, it might seem that the most relevant factor influencing

961 disparity of muscle moment arms is skeletal morphology. While, perhaps unsurprisingly, this

962 appears to generally be correct, limb posture (i.e. behavioural choice of joint orientations) also

963 influences muscle action (e.g. Hutchinson et al., 2005; Hutchinson et al., 2015). In particular, we

964 found notable differences in muscle moment arms for glenohumeral extension/flexion between

965 the reference pose and resting pose for our Mussaurus model, with the SHP, TBS, TBC and BB 
966 switching actions; becoming mixed or purely extensor/flexor in each case (Tables 3, S8). These

967 differences in moment arm values between the reference and the resting pose in a single taxon

968 can be explained in terms of posture and ROM. For example, the SHP displayed a mixed action

969 in the reference pose of Mussaurus, remaining an extensor across some of the glenohumeral

970 joint's ROM, but switching to a flexor at about $-40^{\circ}$ of extension from the reference pose (i.e.

971 more flexed joint angles). However, the resting pose of Mussaurus had a more restricted ROM

$972\left(35^{\circ}\right.$ vs. $80^{\circ}$ in the reference pose) that prevented the SHP from changing into a shoulder flexor

973 (cf. Figs. 6A vs. S5C). The above examples show how deep the influence of an assumed

974 reference pose could be on the action of a single muscle, particularly for an organism in which

975 such a pose is not anatomically likely, emphasising the need for comparisons made in the

976 context of a biologically plausible ('resting') posture, as analysed below.

977

978 Functional differences in the resting pose

979 We focus here on muscles whose actions differed between Crocodylus and Mussaurus in the 980 resting pose. That pose represents a more realistic limb configuration for Mussaurus, allowing 981 us to speculate on underlying causes of such functional changes (e.g. morphology, posture).

982 Furthermore, at the end of this section we compare with data on human forelimb muscle 983 actions, for reasons explained there.

984 It is important to distinguish between muscles that change their action owing to a shift

985 in their paths (in any posture) because of anatomical changes, and muscles that change their

986 actions because of reorientations of the joints that alter muscle paths. Muscles DC and DS

987 provide a good example of this distinction. These two muscles did not change their paths 
988 appreciably from the reference to the resting pose in each taxon, and thus maintained their

989 moment arm patterns, even when moment arms were plotted against a different DOF, to which

990 they are related (Figs. S8, S9). However, the evolution of dinosaurs involved a counterclockwise

991 (as seen from a right lateral view) rotation of the glenohumeral articular face, as previously

992 noted (Jenkins, 1993; Gatesy \& Baier, 2005). This reorientation of the glenoid transformed the 993 functions (i.e. elevation/depression; protraction/retraction) of muscles such as the DC and DS 994 (Figs. 4D-G, 12A).

For example, in Crocodylus, a flexor action could be incurred by muscles to move the 996 humerus perpendicular to the long axis of the glenoid surface (in the same plane as the

997

999

1000

1001

1002 1003

1004

1005

1006

1007

1008

1009 vertebral column; in a craniocaudal arc), whereas an adductor action would move the humerus parallel to the glenoid surface (and perpendicular to the vertebral column; in a dorsoventral arc). In a dinosaur such as Mussaurus, the long axis of the glenohumeral joint is not perpendicular to the vertebral column (as in Crocodylus), but caudoventrally (or obliquely) oriented. In birds such glenohumeral reorientation is taken to an extreme, with the long axis more parallel to the vertebral column (Fig. 12B). This reorientation of the shoulder joint along the dinosaurian lineage means that a flexor movement in a crocodile (or other non-dinosaurian archosaur) would protract the humerus. In a typical dinosaur, conversely, a homologous movement (i.e. a movement perpendicular to the long axis of the glenohumeral joint) would elevate the humerus, and this transformation of flexion (for example) would apply to Mussaurus (Fig. 12).

In the resting pose, SHP was identified as a humeral supinator in Crocodylus but a pronator in Mussaurus, although its origin and insertion sites in both taxa are placed in 
1010 topologically similar areas on the scapula and humerus, respectively. A role in glenohumeral

1011 long axis rotation has not been previously reported for this muscle, to our knowledge. The

1012 cause of this divergence in muscle actions would thus be the morphological disparity of the

1013 humerus between the crocodile and Mussaurus. The humerus of Crocodylus has a narrow

1014 proximal shape in comparison with its shaft, whereas Mussaurus presents an expanded

1015 humeral proximal end, as is typical for all early sauropodomorphs (Galton \& Upchurch, 2004).

1016 Hence the more laterally positioned insertion of the SHP in Mussaurus (Fig. 3) resulted in a

1017 sustained pronator action. Conversely, in Crocodylus, SHP's insertion slightly medial to the

1018 humeral midline (Meers, 2003) resulted in a supinator action.

1019 Other morphology-based differences between our study taxa are clearly caused by

1020 osteological correlates indicating soft tissue attachments, rather than by general bony

1021 geometry. The CBV retained the same shoulder pronator and adductor actions in both taxa (see

1022 also Meers, 2003; Jasinoski, Russell \& Currie, 2006), but differed strikingly around the

1023 extension/flexion axis, being a extensor in Crocodylus and a flexor in Mussaurus (Fig. 6C, Table

1024 3). The protractor and adductor actions of CBV seem to be ancestral for Crocodylia and

1025 presumably Archosauria (Meers, 2003; Jasinoski, Russell \& Currie, 2006; Burch, 2014), but the

1026 derived action in Mussaurus appears to have been incurred by a shift of its path to lie more

1027 cranially on the coracoid (Fig. 2).

1028

Some muscles had different action(s) in at least one degree of freedom in the resting

1029 pose for Crocodylus and Mussaurus that could best be explained by joint ROMs inferred from

1030 our models. TBS was a glenohumeral extensor in Crocodylus, as is ancestral for Archosauria

1031 (Meers, 2003; Jasinoski, Russell \& Currie, 2006; Burch, 2014). However, TBS had a flexor action 
1032 in Mussaurus when the joint was moved beyond $-60^{\circ}$ of extension. As the ROM for

1033 glenohumeral maximal extension was limited to $-60^{\circ}$ in Crocodylus vs $-70^{\circ}$ in Mussaurus, this $103410^{\circ}$ difference in ROM was sufficient to alter the TBS from being a pure extensor to having a 1035 mixed action in the latter taxon (Fig. 6C). This pattern of a restricted extension/flexion ROM 1036 preventing certain muscles in Mussaurus from switching actions appears common (Figs. 6, S8, 1037 S9) and would be sensitive to the accuracy of our ROM estimates.

Not all forelimb muscles in our analysis, however, showed different actions in

Crocodylus and Mussaurus. Such muscles are interesting, too, because they might have had a conservative function across (at least non-avian) Archosauria. For example, DC and DS were the only muscles acting around the glenohumeral joint that preserved the same action in the three DOFs for both Crocodylus and Mussaurus (Table 3), combining glenohumeral supination, flexion and abduction. These qualitatively identical muscle actions (regardless of their functions in the resting pose; Fig. 12) are reflected by the conservative attachment sites of both DS and DC on the lateral scapular blade and proximal humerus in these two taxa and, more generally, in Archosauria (Meers, 2003; Jasinoski, Russell \& Currie, 2006; Langer, Franca \& Gabriel, 2007; Remes, 2008; Suzuki \& Hayashi, 2010; Burch, 2014; Klinkhamer et al., 2017). The conservative action of the deltoid muscle heads is partially congruent with previous studies that qualitatively inferred crocodile forelimb functions (Meers, 2003; Jasinoski, Russell \& Currie, 2006; Allen et al., 2014; Klinkhamer et al., 2017). The TM and TBC, likewise, preserved common shoulder supinator, extensor and abductor actions; whereas the SBS remained a pronator, extensor and 1052 adductor; and the SC kept its action as a supinator, flexor and adductor. 
elbow joint also showed unambiguous actions in Crocodylus and Mussaurus, and more

generally in Archosauria (Meers, 2003; Jasinoski, Russell \& Currie, 2006; Burch, 2014, 2017;

1056

Klinkhamer et al., 2017). The PT muscle was estimated to act as an elbow extensor in

Crocodylus but an elbow flexor in Mussaurus (Table 3; Fig. 8C). The more caudal position of the

1058

1059

1060

1061

1062

1063

1064

1065

1066

1067

1068

1069

1070

1071

1072

1073

1074
PT's origin in the former taxon vs. more cranial in the latter (Fig. 2; Table 1) explains this

difference. Relative differences in the magnitudes of moment arms, such as the larger

normalized values for some muscles (particularly the EDL) acting about the carpus in Crocodylus

(Fig. 9), surely relate to the paths of those muscles around the joints and thus to soft tissue

morphology and osteological influences on it.

However, less straightforward actions were evident for muscles that originated at either

side of the humeral distal condyles, which could experience posture-dependent switches of

their actions (Fig. 8). We found complex actions like these for SU, FU, AR, EDL, ECR and ECU,

which were elbow extensors in Crocodylus but had mixed actions in Mussaurus (see Meers,

2003; Burch, 2014, 2017 for different interpretations), or FDL, which had a mixed action in

Crocodylus but was purely a flexor in Mussaurus. The differences in ROM values between both

taxa seemingly did not affect the actions of these muscles (Tables 3, S8). Instead, the actions of

these muscles were extremely sensitive to placements of their origin sites (Figs. 2, S1). The

main problem resulting from this sensitivity is the uncertainty about the location of the origin

sites in Mussaurus, which are obscured by pitting and other artefacts left by the articular

cartilage. Below, we consider the effects of missing cartilage on our general conclusions about

forelimb biomechanics in Mussaurus. 
Of all forelimb muscles in tetrapods, the actions in humans are the best understood,

1076

1077

1078

1079

1080

1081

1082

1083

1084

1085

1086

1087

1088

1089

1090

1091

1092

1093

1094

1095

particularly using these musculoskeletal modelling frameworks and often in conjunction with validation methods such as magnetic resonance imaging or 'tendon travel' experiments (see references below for an introduction). Although a detailed comparison with Mussaurus (or Crocodylus) is far beyond the scope of this study, general patterns of similarities and differences in muscle actions are evident. First, however, the actions must be considered in light of joint ROMs. Compared with Mussaurus and Crocodylus (Table 2), humans (data from Murray, Delp \& Buchanan, 1995; Murray, Buchanan \& Delp, 2000; Holzbaur, Murray \& Delp, 2005; Rankin \& Neptune, 2012) have much larger total ROMs for extension/flexion ( $\left.120^{\circ}\right)$ and long-axis rotation $\left(\sim 105^{\circ}\right)$ of the shoulder (let alone the highly mobile scapula). Humans also certainly have a greater range of forearm pronation/supination $\left(\sim 160^{\circ}\right.$ in models although far less is used in vivo; closer to $\left.90^{\circ}\right)$. Otherwise, the ROMs of the human shoulder in ab/adduction $\left(\sim 65^{\circ}\right)$ and elbow and wrist in flexion/extension ( $\sim 100$ and $90^{\circ}$ respectively; also $\sim 35^{\circ}$ of wrist ab/adduction or 'deviation') are roughly similar to the archosaurs we studied.

Keeping these similarities and differences in joint ROMs in mind, and focusing mainly on extensor/flexor action, there are interesting similarities. In all three taxa, the deltoid muscles tend to increase their shoulder flexor moment arms with increasing joint flexion, and teres major muscles tend to have shoulder extensor moment arms that peak at moderate angles of shoulder flexion (e.g. Ackland et al., 2008; vs. our Fig. 6). Yet differences in shoulder muscle actions also stand out. For instance, human subscapularis muscles have high flexor moment arms in extended shoulder positions, moving toward extensor action as the shoulder becomes 
1096 strongly flexed (e.g. Ackland et al., 2008), whereas the subscapularis muscles in our archosaur

1097 models are predominantly extensors (Fig. 6).

Around the elbow joint, human and archosaur triceps muscles have more flattened

1099

1100

1101

1102

1103

1104

1105

1106

1107

1108

1109

1110

1111

1112

1113

1114

1115

1116 extensor moment arm vs. joint angle curves, whereas the biceps and brachialis elbow flexors

have pronounced peaks for their elbow flexor moment arms at moderate flexion angles

(Murray, Buchanan \& Delp, 2000; our Fig. 8). Data from humans hint that these consistent

patterns may relate to different usages of the active force-length curves of these muscles:

elbow flexors tend to range far in their relative lengths across the ascending limb and plateau of

that curve, whereas triceps muscles tend to remain closer to the curve's plateau (Murray,

Buchanan \& Delp, 2000: their Fig. 5). In other words, the former muscles may be more

specialized for length change whereas the triceps muscles, even in bipeds (such as

Mussaurus?), may remain specialized for antigravity functions such as high isometric force

production. This speculation deserves testing with more direct measurements in living

archosaurs as well as modelling investigations; moment arms alone are only tantalizing in this

regard. However, other muscles acting around the elbow show some divergent patterns: for

example, the pronator teres is a weak elbow flexor in humans but has a moderate extensor or

more mixed action in our archosaur models (see above), and the very transformed $\mathrm{M}$.

brachioradialis in humans is an elbow flexor with a large moment arm (and capacity for length

change), unlike the corresponding ECR+ECU muscles in our models which are estimated to be

elbow extensors in Crocodylus and mixed in Mussaurus (Murray, Delp \& Buchanan, 1995, their

Fig. 4; vs. our Fig. 8). 

ulnaris) comparable with our models' FDL in terms of anatomy. Their moment arms for flexion increase slightly with wrist flexion angle-but in our archosaur models, these muscles exhibit weaker flexion moment arms with increasing flexion and even switch to extensors at extremely peak at moderate joint angles close to the reference pose, and may switch to flexor action in Crocodylus if the wrist is strongly extended (Gonzalez, Buchanan \& Delp, 1997, their Fig. 4; vs. our Fig. 9A). results from some other animals, especially quadrupedal mammals such as hares and greyhounds (Williams et al., 2007, 2008), reinforcing that some similarities may be generalizations for tetrapods or amniotes whereas others may be specializations particular to mammals or archosaurs (or bipeds). Unfortunately too few data exist, especially for nonmammals, to test these ideas and more focus is needed on collecting new data from experiments and models of various taxa, joints and muscles. We have only made a small step here toward integrating these disparate sources of data to understand the evolution of muscle 1136 actions and functions. 
1139 The general patterns that we present here for moment arm postural changes and muscle

1140 actions (e.g. extensor/flexor/mixed) in Crocodylus and Mussaurus are not influenced by our

1141 choice of segment length as a normalizing metric. However, comparisons of the absolute and

1142 normalized values of moment arms are influenced by the vast differences in morphology and

1143 posture (and phylogenetic divergence times) between our two study taxa. Considering that

1144 relative rather than absolute values were most needed here, and the latter problems of

1145 moment arm comparisons across disparate taxa, we have generally not emphasized those

1146 values of moment arms. As Table S2 shows, the ratios of corresponding segment lengths from

1147 our two models vary little; between 1.9-2 (Mussaurus has relatively longer proximal segments,

1148 especially humerus). These ratios slightly complicate proximodistal comparisons across the

1149 limbs, which were not a focus of our study. More problematically, the ideal normalization

1150 metric would be body mass (to remove size influences), but this is unknown for Mussaurus.

1151 Using the minimal humeral and femoral circumferences of our Mussaurus specimen and

1152 equation 2 from Campione \& Evans (2012), we obtained an estimate of $1486 \mathrm{~kg}$ body mass,

115373.6 times that of our Crocodylus specimen, or 4.13 times if the cube root of body mass were

1154 desired as an approximately linear normalizer (reducing moment arms in metres to

1155 dimensionless units as in this study's main results). The equation used for body mass estimation

1156 has 'error bars' of appreciable size, but our focus was more on qualitative comparisons of

1157 muscle actions than quantitative ones (especially absolute magnitudes except where

1158 exceptional). Additionally, we provide our models, results and normalizing metrics here, so it is

1159 feasible for future studies to inspect the effects of this assumption in more detail if desired. We

1160 do not expect that our conclusions would be considerably altered by using a different 
1161 normalizing metric. If more taxa were included in our analysis, this issue would become more

1162 important to consider, so we raise it here but do not elaborate further. An alternative approach 1163 would be to present ratios of moment arms (e.g. extensor/flexor vs. abductor/adductor) but for

1164 simplicity we did not add this analysis. Studies of human moment arms have suggested that the

1165 ideal normalization metrics may even vary with the relative distance of the origin or insertion

1166 from the joint centre, and thus bone lengths, diameters or circumferences might thus be

1167 insufficient for normalization (Murray, Buchanan \& Delp, 2002).

1168

1169

Sensitivity analysis: Influence of cartilage volume on moment arms at the elbow joint

1170 One of the major challenges inherent to soft tissue reconstructions in extinct archosaurs is the

1171 reliable inference of sites of origin and insertion of muscles, as well as 3D paths between them.

1172 However, some muscles leave notable scars and protuberances on the bone surfaces, and thus

1173 inferences about their existence and locations become less speculative than those muscles for

1174 which no osteological correlates exist (Bryant \& Seymour, 1990; Witmer, 1995). This is relevant

1175 not only for the correct interpretation of the anatomy of the animal, but also has a profound

1176 impact on biomechanical inferences based on the musculoskeletal anatomy (e.g. Hutchinson et

1177 al., 2005). The moment arms of some limb muscles are very sensitive to the inferences made

1178 about muscle attachments and paths, especially insertions. Fortunately, in many cases the

1179 more concentrated nature of insertions (vs. more diffuse nature of proximal origins of muscles,

1180 which tend to taper toward their distal insertions) means that the insertions have clearer scars

1181 and thus locations, thereby sometimes reducing concerns about that sensitivity. Yet as noted

1182 above for the elbow (e.g. distal humerus), missing articular/epiphyseal cartilages is one clear 
1183 case where there is cause for special concern and thus attention to potential sensitivity of the

1184 moment arms of muscles that cross the elbow joint.

1185 The inference of epiphyseal cartilage in extinct archosaur limbs has been the subject of

1186 debate and speculation since the late 1800s (e.g. Owen, 1875; Cope, 1878; Osborn, 1898), with

1187 the main focus of discussions centred on the estimated volume occupied by the original

1188 cartilage cap (Holliday et al., 2010; Bonnan et al., 2013; Reiss \& Mallison, 2014; White et al.,

1189 2015). However, inferences about the impact of cartilage volume in functional studies have

1190 received less attention (but see Gatesy, Bäker \& Hutchinson, 2009; Fujiwara, Taru \& Suzuki,

1191 2010; Tsai \& Holliday, 2014; Taylor \& Wedel, 2013). The lost cartilage during the process of

1192 fossilization in dinosaurs is evident in the simplified epiphyseal surfaces of the long bones.

1193 These missing surfaces also complicate interpretations of musculoskeletal biomechanics

1194 because they affect the assumed length of the segment(s) analyzed and the shape of the

1195 articular facet(s) as well (Holliday et al., 2010; Bonnan et al., 2013). Similarly, functional

1196 analyses dealing with joint articulations of limb bones in archosaurs have mostly focused on

1197 how the absence (or presence) of cartilage can influence the ranges of motion (ROM) of joints

1198 (Mallison, 2010b; Hutson \& Hutson, 2012, 2013, 2014; Reiss \& Mallison, 2014), although some

1199 studies opted for a bone-on-bone analysis, arguing that speculation about cartilage extent was

1200 simply excessive (White et al., 2015). Overall, there is virtually no information on how

1201 unpreserved cartilage volumes may affect muscle function in archosaur limbs. To address this

1202 matter in our musculoskeletal model by testing how the estimated moment arms were

1203 influenced by articular cartilage morphology, we varied the effective cartilage volume of the

1204 epiphyses by altering the wrapping surfaces of muscles crossing the elbow joint. 
Increasing or decreasing the radius of the cylinder that muscles traversing the distal

1206

1207

1208

1209

1210

1211

1212

1213

1214

1215

1216

1217

1218

1219

1220

1221

1222

1223

1224

1225

1226

humeral condyles must wrap around when they contacted it represented an increase/decrease of the epiphyseal cartilage assumed for the elbow joint. Subsequently, the radius of this wrapping cylinder was then increased or decreased by $25 \%$ of its original value (Fig. 13; Table S11), and in each case we recalculated all of the affected muscles' moment arms (Table S10). These changes required some adjustments of muscle points to prevent muscle paths from penetrating the wrapping cylinder (Fig. 13A,C).

Our results from this sensitivity analysis showed that altering the effective cartilage volume at the elbow did not affect the qualitative pattern of moment arms for extensor musculature (Fig. 14). The triceps muscle moment arms showed the same human-like pattern described above (increase of extensor moment arm with flexion past the resting pose, then decrease past $\sim 90^{\circ}$ ). In spite of the similar trajectories of the moment arm curves, altering the volume of the hypothetical cartilage cap did (unsurprisingly) alter moment arm values around the elbow. Reduction of the wrapping surface at the elbow by $25 \%$ of its radius considerably decreased the overall moment arms of extensor muscles. At full elbow extension, moment arms with $25 \%$ reduction of the wrapping surface (i.e. 'cartilage') were smaller by about $0.01 \mathrm{~m}$ from the original values, and almost $0.02 \mathrm{~m}$ smaller than the model with enlarged $(+25 \%)$ wrapping surfaces (Table S10). At full flexion, however, leverage differences increased, involving larger values for the model with increased wrapping surface, as expected (Fig. 14; Table S10). These results were expected because muscle moment arms can be defined as the minimal distance between the line of action of a muscle-tendon complex and the centre of rotation of a joint (e.g. An et al., 1984; Pandy, 1999). On average, moment arms calculated for 
1227 the $25 \%$ reduced 'cartilage cap' experienced a decrease of $15 \%$ of moment arms relative to the

1228 non-altered model's mean moment arm (Table S10), whereas an increase of $25 \%$ to the

1229 'cartilage cap' resulted in a 14\% increase of moment arm values. Hence, reduction of the

1230 cartilage cap (as a wrapping surface) around a given joint should lead to a reduction (although

1231 potentially non-proportional) of the moment arms for that joint. Thus, although consideration

1232 of cartilage volume and estimated shape in ROM analysis deserves scrutiny because of

1233 potential for subjectivity (White et al., 2015), our results demonstrate that missing articular

1234 cartilage will influence muscle moment arm variations, highlighting the importance of

1235 epiphyseal caps for inferences about muscle functions and evolution.

1236

1237 Manus pronation in Mussaurus and the evolution of quadrupedalism in Sauropodomorpha

1238 The biped-quadruped transition in Sauropodomorpha was linked with the dramatic postural

1239 changes that evolved from the smaller sauropodomorph ancestors to the gigantic sauropods

1240 (Upchurch, Barrett \& Galton, 2004). Such changes also involved a series of anatomical

1241 transformations, including increased body mass and a forward shift of the body's centre of

1242 mass (Bates et al., 2016), modification of limb proportions (Wilson, 2002), and successive

1243 addition of sacral vertebrae (Wilson \& Sereno, 1998; Pol, Garrido \& Cerda, 2011), among

1244 others. Furthermore, over the past 15 years, pronation of the manus has been proposed as a

1245 critical anatomical feature associated with the acquisition of quadrupedal locomotion in

1246 different lineages of Dinosauria (Bonnan, 2003; Bonnan \& Senter, 2007; Bonnan \& Yates, 2007;

1247 Maidment \& Barrett, 2012; VanBuren \& Bonnan, 2013; Hutson, 2015). The growing consensus

1248 is that increased manus pronation originated during the early evolution of large-bodied, 
1249 quadrupedal and graviportal sauropodomorphs for improved support against gravity. This

1250 consensus exists in contrast to the evolution of pronation capabilities in extant taxa, including

1251 some lizards and mammals, in which pronation seems to have been correlated with increased

1252 arboreality at small body sizes (Matthew, 1904; Haines, 1958; VanBuren \& Bonnan, 2013;

1253 Hutson, 2015; Hutson \& Hutson, 2017).

1254

1255

1256

1257

1258

1259

1260

1261

1262

1263

1264

1265

1266

1267

1268

1269

1270

Bonnan (2003) and Bonnan \& Yates (2007) hypothesised that at least a semi-pronated

manus in sauropodomorph dinosaurs was facilitated, among others, by the evolution of the craniolateral process of the proximal ulna that accommodated the radius in a cranial (not medial) position relative to the ulna proximally, and in a medial position relative to the ulna distally. Then, the evolution of the characteristic U-shaped manus in eusauropods may have originated in relation to increased pronation (Bonnan \& Yates, 2007, p. 166). In contrast,

Hutson (2015) and Hutson \& Hutson (2015) proposed that the evolution of the craniolateral process of the ulna was a specialization to immobilize the proximal radioulnar joint. Either way, increased pronation of the manus should have aided the forelimbs to generate craniocaudally directed propulsive or braking forces that roughly paralleled the actions of the pes in a parasagittal plane (Bonnan, 2003). Excluding (putatively ancestrally) bipedal forms such as Panphagia and Saturnalia, most non-sauropod sauropodomorphs are hypothesized to have been either facultative quadrupeds or bipeds, although few studies have delved deeply into this topic.

Moreover, although widely cited in the literature, the terms 'active' and 'passive' pronation have been scarcely defined, existing mostly in an implicit fashion (see Bonnan \& Senter, 2007; Bonnan \& Yates, 2007; VanBuren \& Bonnan, 2013). Hutson \& Hutson (2015, 
1271 2017), however, defined active forearm pronation as anteromedial long-axis rotation. Here we

1272 define active pronation as the muscle-driven ability to rotate the manus around its longitudinal 1273 axis, from pronation to supination, by any kind of rearrangements of the antebrachial bones.

1274 Active pronation may facilitate facultative quadrupedalism. Passive pronation implies a manus

1275 fixed into pronation, with no clear ability to supinate, leading to obligate quadrupedalism. Massospondyus had poor abilities for quadrupedal locomotion (thus favouring bipedalism) based on the restricted ROMs of their limb joints and the morphology of their radius and ulna pronation. Additionally, ROM analysis performed on a 3D skeleton of Plateosaurus showed that radius rotation around the ulna was impossible, mainly because of its oval-shaped (not circular) proximal end, precluding pronational capabilities and thus quadrupedal locomotion (Mallison, 2010a; Mallison, 2010b). Nonetheless, a permanently semi-pronated manus was not ruled out for Plateosaurus (Mallison, 2010b). In contrast to this, active pronation has been reported in some therian clades with an oval radial epiphysis (Hutson \& Hutson, 2017), implying that rotation of the radius against the ulna should be analysed considering not just one parameter, such as the shape of the proximal radius (see also VanBuren \& Bonnan, 2013), but also a broader range of traits. In contrast, a permanently semi-pronated manus is inferred to have been present in Melanorosaurus (Bonnan \& Yates, 2007), a sauropodomorph closely related to Sauropoda (Yates, 2007; Pol et al., 2011; Otero et al., 2015). In the latter studies, a semi-pronated manus was concluded to have evolved at least in sauropodomorphs close to Sauropoda, at the base of 
1293 the 'quadrupedal clade'. This clade retained other clearly 'prosauropod-like' forelimb features

1294 (e.g. an arched metacarpus, three manus claws, and a medially divergent pollex), indicating a 1295 potential decoupling of manus shape and quadrupedalism. Other features hint at a functional

1296 connection between forelimb morphology (e.g. presence of a craniolateral process on the ulna)

1297 and manus shape (i.e. presence of an arched, rather than bundled, metacarpus) (Bonnan \&

1298 Yates, 2007). Regardless, how the forelimbs of early sauropodomorphs were used for functions

1299 other than purely locomotion has hitherto not been convincingly addressed, and the functional

1300 steps that ultimately produced the derived locomotor mechanisms present in Sauropoda

1301 remain obscure, deserving testing with a wider sample of taxa.

The forelimb of Mussaurus patagonicus is particularly interesting because it displays a

1303

1304

1305

1306

1307

1308

1309

1310

1311

1312

1313 combination of plesiomorphic and derived features. For example, it has sauropodomorph

plesiomorphies such as expanded humeral epiphyses, a metacarpus that is arranged into a gentle arch, and a robust metacarpal I with a medially divergent pollex (Otero \& Pol, 2013).

Contrastingly, the evolution of an incipient craniolateral process of the proximal ulna (indicating a rearrangement of the radius relative to the ulna; Bonnan \& Yates, 2007) is a derived feature in Mussaurus, shared with other sauropodiforms (e.g. Aardonyx, Sefapanosaurus,

Melanorosaurus) and sauropods. Moreover, Mussaurus is phylogenetically placed at the base of the sauropodiform clade (Otero \& Pol, 2013; McPhee et al., 2015; Otero et al., 2015), constituting an intermediate taxon to test pronation capabilities between the plesiomorphic pattern present in non-sauropodiform sauropodomorphs (i.e. Massospondylus, Plateosaurus) and the derived pattern inferred for the closest relatives of Sauropoda (i.e. Melanorosaurus). 
To estimate the potential for manus pronation in Mussaurus, we used our 3D

1315

1316

1317

1318

1319

1320

1321

1322

1323

1324

1325

musculoskeletal model to evaluate how the radius might have been accommodated against the ulna and which antebrachial configurations Mussaurus could have adopted in order to achieve some amounts of manus pronation. Recent studies demonstrated that the morphology of the radius is an important determinant of pronation capabilities, such as the presence of radial shaft curvature (allowing the radius to cross the ulna) and a rounded proximal articular face (permitting the radius to rotate around the proximal end of the ulna during active pronation); a condition typical of extant mammals (VanBuren \& Bonnan, 2013; Hutson \& Hutson, 2017).

Nonetheless, the presence of a mediolaterally expanded radial head and the absence of radial shaft curvature may have precluded active manus pronation in most dinosaurs (VanBuren \& Bonnan, 2013) (and perhaps other archosaurs; e.g. Hutson, 2015). Moreover, another feature would have prevented active manus pronation specifically in sauropodomorph dinosaurs. The distal end of the radius of several sauropodomorphs across the transition to Sauropoda had a prominent tubercle on the caudodistal surface, which was suggested to be an osteological correlate of the radioulnar ligament's attachment (Remes, 2008, Yates et al., 2010, Otero \& Pol, 2013, McPhee et al., 2014; Otero et al., 2015). This caudodistal tubercle of the radius is a feature characteristic of basal sauropodiforms such as Mussaurus, Aardonyx, Sefapanosaurus, Melanorosaurus and Antetonitrus (Fig. 15; McPhee et al., 2014; Otero et al., 2015), and it is also present in the basal sauropod Tazoudasaurus (Allain \& Aquesbi, 2008: Fig. 22).

Digital manipulation of our 3D model of Mussaurus in our ROM analyses showed that there was limited possibility of radial movement against the ulna both proximally and distally. The elliptical proximal surface of the radius precluded pronation/supination and the distal 
1336 tubercle would have locked the distal radius and ulna, placing the former cranial to the latter.

1337 Furthermore, the radius of Mussaurus is rather straight, making radial crossing around the ulna 1338 impossible. Considering these constraints, the most likely way to articulate the radius and ulna 1339 in an anatomically plausible way was with the radius cranial to the ulna proximally, and slightly 1340 medially distally, as previously suggested by Bonnan (2003). Nonetheless, with this antebrachial 1341 configuration, we infer that appreciable manus pronation (via radioulnar rotation) was not 1342 possible in Mussaurus. Thus, the only way to achieve some degree of pronation in Mussaurus 1343 was through pronation (internal/medial rotation) of the whole antebrachium as a single unit 1344 (i.e. around the elbow joint) by up to about $-30^{\circ}$ (Table 2). With this configuration, some degree 1345 of manus pronation might have been achievable, although far from the full pronation of the manus that might be consistent with permanently quadrupedal locomotion (Fig. 16). This does not mean that Mussaurus actually did perform pronation in this way, but it is a possibility, considering that the articular surfaces of the distal humerus and proximal radius seem to allow it (albeit cartilage shape is unknown). Considering that crocodylians appear able to conduct some similar long-axis rotation (Baier and Gatesy, 2013), this is not an outrageous proposition. the inference that mobility of the radius against the ulna was severely restricted in most nonsauropodiform sauropodomorph dinosaurs, making active pronation of the manus through antebrachial rotation highly unlikely. Nonetheless, our ROM analysis showed that active semipronation might have been possible in Mussaurus through pronation of the whole antebrachium at the elbow $\left(-30^{\circ}\right)$. This potential pronation ability constitutes a novel, but tentative, finding for basal sauropodomorphs, consistent with the inference that facultative 
1358 quadrupedalism should not be ruled out for this taxon (and perhaps close relatives), although

1359 obligate quadrupedalism was unlikely.

1360 However, active pronation would presumably have been muscle-driven; and thus

1361 moment arms would be important for driving such motions or controlling postures. Our

1362 moment arm analysis for pronation/supination around the elbow (Fig. S10) was interesting in

1363 two ways in this regard. First, we found that only the FU muscle could pronate the elbow joint

1364 (increasing its pronator moment arm as the elbow became less pronated); all other

1365 antebrachial muscles were consistently supinators. Second, almost all supinator muscles acting

1366 around the elbow (except the EDL, ECR and ECU) had maximal supinator moment arms at $-30^{\circ}$

1367 pronation; reducing with increased elbow supination. It is not clear what pronation/supination

1368 moments the elbows of Mussaurus would have needed to support if used in an antigravity role

1369 during quadrupedal locomotion, but our model indicates a potentially overall greater leverage

1370 of the forelimb (for actions of pronation or supination) in a non-pronated orientation; i.e. closer

1371 to $0^{\circ}$ (Table S12). Note that the elbows of Mussaurus in an antigravity role almost certainly

1372 would have had to sustain extensor muscle actions (e.g. Triceps muscle group activity) and the

1373 activations of these muscles would have created supinator moments around the elbow that the

1374 FU muscle alone might have had difficulty opposing to maintain a semi-pronated posture (i.e.

1375 when its moment arms were minimal but its antagonists' were maximal). Together, this

1376 evidence does not strongly favour the ability of Mussaurus to actively pronate its elbow joints,

1377 but it remains a possibility based on our ROM results in particular. As we cautioned above,

1378 however, these inferences are strongly contingent on our assumptions and conclusions about

1379 elbow joint morphology and articular cartilage in Mussaurus. 
The evolution of a pronated manus has been postulated to have begun at least prior to

1381

1382

1383

1384

1385

1386

1387

1388

1389

1390

1391

1392

1393

1394

1395

the rise of sauropods, at the origin of the quadrupedal sauropodiform clade (i.e.

Melanorosaurus, Bonnan \& Yates, 2007; Yates et al., 2010). Aardonyx, a basal member relative to Melanorosaurus outside the quadrupedal clade, was proposed to have had some features that preceded quadrupedal locomotion in sauropodomorphs, such as an incipient craniolateral process of the ulna and a rather straight femoral shaft (Yates et al., 2010), but the question of how much earlier this evolution began has remained unresolved. We conclude, considering past studies as well as our new data for Mussaurus, that full, passive manus pronation was not present at the base of Sauropodiformes (sensu Sereno, 2007), but instead much closer to the origin of Sauropoda than previously thought (see also Yates et al., 2010). However, we cannot exclude some capacity for active pronation in Mussaurus and presumably some other sauropodiforms, as a potential intermediate state in this transformational series of forelimb function. One alternative would be that quadrupedalism did not merely evolve once in the sauropodomorph lineage, but rather that mosaic evolution in early Sauropodiformes resulted in some taxa tending to use quadrupedalism more often than others did. Ultimately, reconstruction of the origin, and perhaps stepwise acquisition, of manus pronation in Sauropodomorpha will depend upon further analyses using not only qualitative, descriptive approaches but also quantitative, explicitly three-dimensional methods such as the one adopted here.

\section{CONCLUSIONS}


1401 We have presented the first quantitative evaluation of forelimb muscle actions in a

1402 sauropodomorph dinosaur, and combined this with assessments of joint mobility and

1403 phylogenetic inferences. Comparisons made with Crocodylus, which represents a mode of

1404 locomotion that is closer to the presumed ancestral state for Archosauria, frame our study in a

1405 broader context to better understand major locomotor shifts in the sauropodomorph line

1406 within Archosauria, including a review of the major literature.

1407 Analysis of moment arms revealed that, first: major differences of muscle actions

1408 between Crocodylus and Mussaurus are evident at the glenohumeral joint, and such changes

1409 are correlated with the morphology of the scapula and the orientation of the glenohumeral

1410 articulation in both taxa (supporting the inference that many of these changes occurred from

1411 Archosauria to Dinosauria/Sauropodomorpha). Second, forelimb posture has great impact on

1412 moment arm values, more so in many cases than morphology. Third, our analysis of reference

1413 versus resting pose in the studied taxa demonstrated how extensive the influence of such poses

1414 could be on the action of a single muscle, particularly for an organism in which that pose is not

1415 anatomically likely (such as Mussaurus), requiring the need for comparisons made in a context

1416 of biologically plausible posture (i.e. resting pose). Fourth, caution is warranted when

1417 comparing organisms with shifted joint coordinate systems, like Crocodylus (sprawled

1418 limb/vertical scapula) and Mussaurus (erect limb/caudoventrally inclined scapula), in which the

1419 same homologous movement, like extension/flexion (i.e. the humerus moving perpendicular to

1420 the long axis of the glenoid), actually corresponds to protraction/retraction (i.e. the humerus

1421 moving in a cranial/caudal direction relative to the ground) in the former and

1422 elevation/depression in the latter (i.e. the humerus moving dorsal/ventral relative to the 
1423 ground, see Fig. 4), as raised by Gatesy \& Baier (2005). Fifth, sensitivity analysis conducted on

1424 Mussaurus' elbow joint confirms that more extensive cartilage volume would increase the 1425 moment arms of elbow extensor muscles, in particular.

Finally, habitual quadrupedalism in Mussaurus is not supported by our joint ROM analysis,

1427 in which glenohumeral protraction was found to be severely restricted. Additionally, some

1428 small amount of active pronation of the manus might have been possible in Mussaurus, and

1429 perhaps in other earlier sauropodomorphs, via long-axis rotation at the elbow to achieve semi-

1430 pronation of the whole antebrachium (not rotation of the radius around the ulna, as previously

1431 thought). In summary, then, the rise of quadrupedalism in Sauropoda would be linked not only

1432 to manus pronation, which should have occurred very close to the node Sauropoda. This

1433 quadrupedalism was also enabled by shifting forelimb morphology as a whole, allowing larger

1434 extension/flexion excursions of the glenohumeral joint and a more columnar forelimb posture.

1435 Our open modelling methods allow others to inspect and build upon these findings. Further

1436 methodological progress in refining how joint ROM is estimated and its biological implications

1437 for certain behaviours, combined with data from how living animals move their joints with

1438 muscles (especially involving how muscle areas and lengths contribute to joint moments and

1439 locomotion), is needed to build consensus in this field, particularly regarding the evolution of

1440 manus pronation vs. quadrupedalism in Archosauria.

1441

1442 ACKNOWLEDGMENTS

1443 N. Muñoz (Museo de La Plata) digitized the forelimb of Mussaurus. Three-dimensional models 1444 of Mussaurus were possible thanks to the freely available software Meshlab (3D-CoForm 
1445 project) and 3D Studio (Autodesk Education Community). We thank Kent Vliet and David Kledzik

1446 for providing the crocodile specimen for study and Larry Witmer for the use of his

1447 laboratoryand access to a CT scanner for imaging that specimen. Reviews by Susannah

1448 Maidment and Joel Hutson greatly improved the manuscript.

1449

1450

\section{REFERENCES}

1451

1452

1453

1454

1455

Ackland DC, Pak P, Richardson M, Pandy M.G. 2008. Moment arms of the muscles crossing the anatomical shoulder. Journal of Anatomy 213:383-390.

Allain R, Aquesbi N. 2008. Anatomy and phylogenetic relationships of Tazoudasaurus naimi (Dinosauria, Sauropoda) from the late Early Jurassic of Morocco. Geodiversitas 30:345424.

Allen V, Paxton H, Hutchinson JR. 2009. Variation in center of mass estimates for extant sauropsids and its importance for eeconstructing inertial properties of extinct archosaurs. Anatomical Record 292:1442-461.

Allen V, Molnar J, Parker W, Pollard A, Nolan G, Hutchinson JR. 2014. Comparative architectural properties of limb muscles in Crocodylidae and Alligatoridae and their relevance to divergent use of asymmetrical gaits in extant Crocodylia. Journal of Anatomy 225:569582.

An KN, Takahashi K, Harrigan TP, Chao,EY. 1984. Determination of muscle orientations and moment arms. Journal of Biomechanical Engineering 106: 280-282. 
1465 Arnold P, Fischer MS, Nyakatura JA. 2014. Soft tissue influence on ex vivo mobility in the hip of 1466 Iguana: comparison with in vivo movement and its bearing on joint motion of fossil sprawling tetrapods. Journal of Anatomy 225:31-41.

1468

1469

1470

1471

1472

1473

1474

1475

1476

1477

1478

1479

1480

1481

1482

1483

1484

Baier DB, Gatesy SM. 2013. Three-dimensional skeletal kinematics of the shoulder girdle and forelimb in walking Alligator. Journal of Anatomy 223:462-473.

Baier DB, Gatesy SM, Dial KP. 2013. Three-dimensional, high-resolution skeletal kinematics of the avian wing and shoulder during ascending flappingflight and uphill flap-running. PLOS ONE 8(5):e63982.

Bates KT, Manning PL, Vila V, Hodgetts D. 2008. Three-dimensional modelling and analysis of dinosaur trackways. Palaeontology 51:999-1010.

Bates KT, Schachner ER. 2012. Disparity and convergence in bipedal archosaur locomotion. Journal of the Royal Society Interface 9:1339-1353.

Bates KT, Benson RBJ, Falkingham PL. 2012. A computational analysis of locomotor anatomy and body mass evolution in Allosauroidea (Dinosauria: Theropoda). Paleobiology 38:486-507.

Bates KT, Mannion PD, Falkingham PL, Brussatte SL, Hutchinson JR, Otero A, Sellers WI, Sullivan C, Stevens KA, Allen V. 2016. Temporal and phylogenetic evolution of the sauropod dinosaur body plan. Royal Society Open Science 3:150636.

Biewener AA. 1989. Scaling body support in mammals: limb posture and muscle mechanics. Science 245:45-48. 
1485 Blob RW, Biewener AA. 2001. Mechanics of limb bone loading during terrestrial locomotion in

1486

1487

1488

1489

1490

1491

1492

1493

1494

1495

1496

1497

1498

1499

1500

1501

1502

1503

1504

the green iguana (Iguana iguana) and American alligator (Alligator mississippiensis). Journal of Experimental Biology 204:1099-1122.

Bonaparte JF, Vince M. 1979. El hallazgo del primer nido de Dinosaurios Triásicos (Saurischia, Prosauropoda), Triásico Superior de Patagonia, Argentina. Ameghiniana 16:173-182.

Bonnan MF. 2003. The evolution of manus shape in sauropod dinosaurs: implications for functional morphology, forelimb orientation and sauropod phylogeny. Journal of Vertebrate Paleontology 23:595-613.

Bonnan MF, Senter P. 2007. Were the basal sauropodomorph dinosaurs Plateosaurus and Massospondylus habitual quadrupeds? Special Papers in Palaeontology 77:139-155.

Bonnan MF, Yates AM. 2007. A new description of the forelimb of the basal sauropodomorph Melanorosaurus: implications for the evolution of pronation, manus shape and quadrupedalism in sauropod dinosaurs. Special Papers in Palaeontology 77:157-168.

Bonnan MF, Wilhite DR, Masters SL, Yates AM, Gardner CK, Aguiar A. 2013. What lies beneath: sub-articular long bone shape scaling in eutherian mammals and saurischian dinosaurs suggests different locomotor adaptations for gigantism. PLOS ONE 8(10):e75216.

Brusatte SL, Nesbitt SJ, Irmis RB, Butler RJ, Benton MJ, Norell MA. 2010. The origin and early radiation of dinosaurs. Earth-Science Reviews 101:68-100.

Bryant HN, Seymour KL. 1990. Observations and comments on the reliability of muscle reconstruction in fossil vertebrates. Journal of Morphology 206:109-117. 
1505 Burch SH. 2014. Complete forelimb myology of the basal theropod dinosaur Tawa hallae based 1506 on a novel robust muscle reconstruction method. Journal of Anatomy 225:271-297.

1507

1508

1509

1510

1511

1512

1513

1514

1515

1516

Burch SH. 2017. Myology of the forelimb of Majungasaurus crenatissimus (Theropoda, Abelisauridae) and the morphological consequences of extreme limb reduction. Journal of Anatomy 231:515-531.Campione NE, Evans DC. 2012. A universal scaling relationship between body mass and proximal limb bone dimensions in quadrupedal terrestrial tetrapods. BMC Biology 10:60.

Carrano MT. 1998. Locomotion in non-avian dinosaurs: integrating data from hindlimb kinematics, in vivo strains, and bone morphology. Paleobiology 24:450-469.

Carrano MT. 2000. Homoplasy and the evolution of dinosaur locomotion. Paleobiology 26:489512.

Carrano MT. 2005. The evolution of Sauropod locomotion: morphological diversity of a secondarily quadrupedal radiation. In: Curry-Rogers CA, Wilson JA, eds. The Sauropods: Evolution and Paleobiology. Berkeley: University of California Press, 229-249.

Carrano MT, Biewener AA. 1999. Experimental alteration of limb posture in the chicken (Gallus gallus) and its bearing on the use of birds as analogs for dinosaur locomotion. Journal of Morphology 240:237-249.

Coombs Jr, WP. 1978. Forelimb muscles of the Ankylosauria (Reptilia, Ornithischia). Journal of Paleontology 52:642-657. 
1524 Cooper MR. 1981. The prosauropod Dinosaur Massospondylus carinatus Owen from Zimbabwe:

1525

1526

1527

1528

1529

1530

1531

1532

1533

1534

1535

1536

1537

1538

1539

1540

1541

1542

1543
Its biology, mode of life and phylogenetic significance. Occasional Papers of the Natural Museum of Rhodesia, B, Natural Sciences 6:689-840.

Cope ED. 1878. On the Saurians recently discovered in the Dakota Beds of Colorado. American Naturalist 12:71-85.

Delp SL, Loan JP, Hoy MG, Zajac FE, Topp EL, Rosen JM. 1990. An interactive graphics-based model of the lower extremity to study orthopaedic surgical procedures. IEEE Transactions in Biomedical Engineering 37:757-767.

Delp SL, Loan JP. 1995. A graphics-based software system to develop and analyze models of musculoskeletal structures. Computers in Biology and Medicine 25:21-34.

Delp SL, Loan JP. 2000. A computational framework for simulating and analysing human and animal movement. IEEE Computing in Science and Engineering 2:46-55.

Dial KP. 2003. Wing-assisted incline running and the evolution of flight. Science 299:402-404.

Dodson P, Farlow JO. 1997. The forelimb carriage of ceratopsid dinosaurs. In: Wolberg DL, Stump E, Rosenberg GD, eds. Dinofest International: proceedings of a symposium held at Arizona State University. Philadelphia: Academy of Natural Sciences, 393-398.,

Fraas E. 1913. Die neuesten Dinosaurierfunde in der schwäbischen Trias. Naturwissenschaften 45:1097-1100.

Fujiwara S, Taru H, Suzuki D. 2010. Shape of articular surface of crocodilian (Archosauria) elbow joints and its relevance to sauropsids. Journal of Morphology 271:883-896. 
1544 Fujiwara S, Hutchinson JR. 2012. Elbow joint adductor moment arm as an indicator of forelimb

1545

1546

1547

1548

1549

1550

1551

1552

1553

1554

1555

1556

1557

1558

1559

1560

1561

1562

1563 posture in extinct quadrupedal tetrapods. Proceedings of The Royal Society B 279:25612570.

Galton PM. 1990. Basal sauropodomorpha-Prosauropoda. In: Weishampel DB, Dodson P, Osmólska H, eds. The Dinosauria. Berkeley: University of California Press, 320-344.

Galton PM, Upchurch P. 2004. Prosauropoda. In: Weishampel DB, Dodson P, Osmólska H, eds. The Dinosauria. Berkeley: University of California Press, 232-258.

Gatesy SM. 1990. Caudofemoral musculature and the evolution of theropod locomotion. Paleobiology 16:170-186.

Gatesy SM. 1991. Hind limb movements of the American alligator (Alligator mississippiensis) and postural grades. Journal of Zoology 224:577-588.

Gatesy SM, Middleton KM. 1997. Bipedalism, flight and the evolution of theropod locomotor diversity. Journal of Vertebrate Paleontology 17:308-329.

Gatesy SM, Baier DB. 2005. The origin of the avian flight stroke: a kinematic and kinetic perspective. Paleobiology 31:382-399.

Gatesy SM, Bäker M, Hutchinson JR. 2009. Constraint-based exclusion of limb poses for reconstructing theropod dinosaur locomotion. Journal of Vertebrate Paleontology 29:535-544.

González RV, Buchanan TS, Delp SL. 1997. How muscle architecture and moment arms affect wrist flexion-extension moments. Journal of Biomechanics 30:705-712. 
1564 Gauthier JA, Nesbitt SJ, Schachner ER, Bever GS, Joyce WG. 2011. The bipedal stem crocodilian

1565

1566

1567

1568

1569

1570

1571

1572

1573

1574

1575

1576

1577

1578

1579

1580

1581

1582

Poposaurus gracilis: inferring function in fossils and innovation in archosaur locomotion. Bulletin of the Peabody Museum of Natural History 52:107-126.

Haines RW. 1958. Arboreal or terrestrial ancestry of placental mammals. Quarterly Review of Biology 33:1-23.

Harris JD. 2004. Confusing dinosaurs with mammals: tetrapod phylogenetics and anatomical terminology in the world of homology. The Anatomical Record 281A:1240-1246.

Holliday CM, Ridgely RC, Sedlmayr JC, Witmer LM. 2010. Cartilaginous epiphyses in extant archosaurs and their implications for reconstructing limb function in dinosaurs. PLOS ONE 5(9):e13120.

Holzbaur KR, Murray WM, Delp SL. 2005. A model of the upper extremity for simulating musculoskeletal surgery and analyzing neuromuscular control. Annals of Biomedical Engineering: 33:829-840.

Hutchinson JR, Gatesy SM. 2000. Adductors, abductors, and the evolution of archosaur locomotion. Paleobiology 26:734-751.

Hutchinson JR, Anderson FC, Blemker SS, Delp SL. 2005. Analysis of hindlimb muscle moment arms in Tyrannosaurus rex using a three dimensional musculoskeletal computer model: implications for stance, gait and speed. Paleobiology 31:676-701.

Hutchinson JR, Gatesy SM. 2006. Beyond the bones. Nature 440:292-294. 
1583 Hutchinson JR, Miller C, Fritsch G, Hildebrandt T. 2008. The anatomical foundation for

1584

1585

1586

1587

1588

1589

1590

1591

1592

1593

1594

1595

1596

1597

1598

1599

1600

1601

1602 multidisciplinary studies of animal limb function: examples from dinosaur and elephant limb imaging studies. In: Fritsch G, Frey R. Anatomical imaging: towards a new morphology). Berlin: Springer, 23-38.

Hutchinson JR, Allen V. 2009.The evolutionary continuum of limb function from early theropods to birds. Naturwissenschaften 96:423-448.

Hutchinson JR, Rankin JW, Rubenson J, Rosenbluth KH, Siston RA, Delp SL. 2015. Musculoskeletal modelling of an ostrich (Struthio camelus) pelvic limb: influence of limb orientation on muscular capacity during locomotion. PeerJ 3:e1001 DOI 10.7717/peerj.1001

Hutson JD. 2015. Quadrupedal dinosaurs did not evolve fully pronated forearms: New evidence from the ulna. Acta Palaeontologica Polonica 60:599-610.

Hutson JD, Hutson KN. 2012. A test of the validity of range of motion studies of fossil archosaur elbow mobility using repeated-measures analysis and the extant phylogenetic bracket. Journal of Experimental Biology 215:2030-2038.

Hutson JD, Hutson KN. 2013. Using the American alligator and a repeated-measures design to place constraints on in vivo shoulder joint range of motion in dinosaurs and other fossil archosaurs. Journal of Experimental Biology 216:275-284.

Hutson JD, Hutson KN. 2014. A repeated-measures analysis of the effects of soft tissues on wrist range of motion in the extant phylogenetic bracket of dinosaurs: Implications for the 
1603

1604

1605

1606

1607

1608

1609

1610

1611

1612

1613

1614

1615

1616

functional origins of an automatic wrist folding mechanism in Crocodilia. Anatomical Record 297:1228-1249.

Hutson JD, Hutson KN. 2015. An examination of forearm bone mobility in Alligator mississippiensis (Daudin, 1802) and Struthio camelus Linnaeus, 1758 reveals that Archaeopteryx and dromaeosaurs shared an adaptation for gliding and/or flapping. Geodiversitas 37:325-344

Hutson JD, Hutson KN. 2017. An investigation of the locomotor function of therian forearm pronation provides renewed support for an arboreal, chameleon-like evolutionary stage. Journal of Mammalian Evolution 24:159-177.

Jaekel O. 1910. Die Fussstellung und Lebensweise der grossen Dinosaurier. Zeitschrift der Deutschen Geologischen Gesellschaft, Monatsberichte 62:270-277.

Jasinoski SC, Russell AP, Currie PJ. 2006. An integrative phylogenetic and extrapolatory approach to the reconstruction of dromaeosaur (Theropoda: Eumaniraptora) shoulder musculature. Zoological Journal of The Linnean Society 146:301-344.

Jenkins FAJ. 1993. The evolution of the avian shoulder joint. American Journal of Science 293a:253-267.

Johnson RE, Ostrom JH. 1995. The forelimb of Torosaurus and an analysis of the posture and gait of ceratopsian dinosaurs. In: Thomason JJ, ed. Functional morphology of vertebrate paleontology. Cambridge: Cambridge University Press, 205-218. 
1622 Kambic RE, Roberts TJ, Gatesy SM. 2017. 3-D range of motion envelopes reveal interacting

1623

1624

1625

1626

1627

1628

1629

1630

1631

1632

1633

1634

1635

1636

1637

1638

1639

1640

degrees of freedom in avian hind limb joints. Journal of Anatomy. DOI:

10.1111/joa.12680

Klinkhamer AJ, Wilhite DR, White MA, Wroe S. 2017. Digital dissection and three- dimensional interactive models of limb musculature in the Australian estuarine crocodile (Crocodylus porosus). PLOS ONE 12(4):e0175079.

Langer MC, Franca MAG, Gabriel S. 2007. The pectoral girdle and forelimb anatomy of the stem sauropodomorph Saturnalia tupiniquim (Late Triassic, Brazil). Special Papers in Palaeontology 77:113-137.

Langer MC, Ezcurra MD, Bittencourt JS, Novas FE. 2010. The origin and early evolution of dinosaurs. Biological Reviews 85:55-110.

Maidment SCR, Barrett PM. 2011. The locomotor musculature of basal ornithischian dinosaurs. Journal of Vertebrate Paleontology 31:1265-1291.

Maidment SCR, Barrett PM. 2012. Does morphological convergence imply functional similarity? A test using the evolution of quadrupedalism in ornithischian dinosaurs. Proceedings of the Royal Society B: Biological Sciences 279:3765-3771.

Maidment SCR, Bates KT, Falkingham PL, Vanburen C, Arbour V, Barrett PM. 2014. Locomotion in ornithischian dinosaurs: an assessment using three-dimensionalcomputational modelling. Biological Reviews 89:588-617. 
1641 Mallison H. 2010a. The digital Plateosaurus I: body mass, mass distribution, and posture

1642

1643

1644

1645

1646

1647

1648

1649

1650

1651

1652

1653

1654

1655

1656

1657

1658

1659 assessed using CAD and CAE on a digitally mounted complete skeleton. Palaeontologia Electronica 13:1-26.

Mallison H. 2010b. The digital Plateosaurus II: an assessment of the range of motion of the limbs and vertebral column and of previous reconstructions using a digital skeletal mount. Acta Palaeontologica Polonica 55:433-458.

Matthew WD. 1904. The arboreal ancestry of the Mammalia. The American Naturalist 38:811818.

McPhee BW, Yates AM, Choiniere JN, Abdala F. 2014. The complete anatomy and phylogenetic relationships of Antetonitrus ingenipes (Sauropodiformes, Dinosauria): implications for the origins of Sauropoda. Zoological Journal of the Linnean Society 171:151-205.

McPhee BW, Choiniere JN, Yates AM, Viglietti PA. 2015. A second species of Eucnemesaurus Van Hoepen, 1920 (Dinosauria, Sauropodomorpha): new information on the diversity and evolution of the sauropodomorph fauna of South Africa's lower Elliot Formation (latest Triassic). Journal of Vertebrate Paleontology 35:e980504.

Meers MB. 2003. Crocodylian forelimb musculature and its relevance to archosauria. Anatomical Record 274:892-916.

Murray WM, Delp SL, Buchanan TS. 1995. Variation of muscle moment arms with elbow and forearm position. Journal of Biomechanics 28:513517-515525. 
1660 Murray WM, Buchanan TS, Delp SL. 2000. The isometric functional capacity of muscles that 1661 cross the elbow. Journal of Biomechanics 33:943-952.

1662 1663
Murray WM, Buchanan TS, Delp SL. 2002. Scaling of peak moment arms of elbow muscles with upper extremity bone dimensions. Journal of Biomechanics 35:19-26.

Norman DB. 1980. On the ornithischian dinosaur Iguanodon bernissartensis from Belgium. Mém. Inst. Roy. Sci. Nat. Belg. 178:1-103.

Norman DB. 1986. On the anatomy of Iguanodon atherfieldensis (Ornithischia: Ornithopoda). Bull. Inst. Roy. Sci. Nat. Belg. Sci. Terre 56:281-372.

Nyakatura JA, Andrada E, Curth S, Fischer MS. 2014. Bridging "Romer's Gap": limb mechanics of an extant belly-dragging lizard inform debate on tetrapod locomotion during the early carboniferous. Journal of Evolutionary Biology 41:175-190.

Osborn H. 1898. Additional characters of the great herbivorous dinosaur Camarasaurus. Bulletin American Museum of Natural History 19:219-233.

Ostrom JH. 1974. The pectoral girdle and forelimb function of Deinonychus (Reptilia: Saurischia): a correction. Postilla 165:10-14.

Otero A, Pol D. 2010. Postcranial anatomy and phylogenetic relationships of Mussaurus patagonicus (Dinosauria, Sauropodomorpha). Journal of Vertebrate Paleontology 33:1138-1168. 
1678 Otero A, Pol D, Powell J. 2012. Phylogenetic relationships of Mussaurus patagonicus: testing the 1679 effect of ontogenetically variable characters on three topology. Journal of Vertebrate 1680 Paleontology 33 (Suppl.):153R.

1681

1682

1683

1684

1685

1686

1687

1688

1689

1690

1691

1692

1693

1694

1695

1696

Otero A, Krupandan E, Pol D, Chinsamy A, Choiniere J. 2015. A new basal Sauropodiform from South Africa and the phylogenetic relationships of basal sauropodomorphs. Zoological Journal of the Linnean Society 174:589-634.

Otero A, Allen V, Pol D, Hutchinson JR. 2017a. Crocodylus musculoskeletal models. Figshare. doi: 10.6084/m9.figshare.49286960tero A, Allen V, Pol D, Hutchinson JR. 2017b. Mussaurus musculoskeletal models. Figshare. doi:10.6084/m9.figshare.4928684

Owen R. 1875. Monographs of the British Fossil Reptilia of the Mesozoic Formations. Part II. (Genera Bothriospondylus, Cetiosaurus, Omosaurus). Palaeontographical Society Monographs 29:15-93.

Padian K, Li C, Pchelnikova J. 2010. The trackmaker of Apatopus (Late Triassic, North America): implications for the evolution of archosaur stance and gait. Palaeontology 53:175-189.

Pandy MG. 1999. Moment arm of a muscle force. Exercise and Sport Science Reviews 27:79-118

Parrish MJ. 1986. Locomotor adaptations in the hindlimb and pelvis of the Thecodontia. Hunteria 1:1-35.

Paul GS, Christiansen P. 2000. Forelimb posture in neoceratopsian dinosaurs: implications for gait and locomotion. Paleobiology 26:450-465. 
1697 Pierce SE, Clack JA, Hutchinson JR. 2012. Three-dimensional limb joint mobility in the early $1698 \quad$ tetrapod Ichthyostega. Nature 486:523-526.

1699 Pol D, Garrido A, Cerda IA. 2011. A new nauropodomorph ninosaur from the Early Jurassic of 1700 Patagonia and the origin and evolution of the sauropod-type sacrum. PLOS ONE 1701 6(1):e14572.

Rankin JW, Neptune RR. 2012. Musculotendon lengths and moment arms for a threedimensional upper-extremity model. Journal of Biomechanics 45:1739-1744. Experimental Biology 211:2735-2751.

Romer AS. 1923. Crocodilian pelvic muscles and their avian and reptilian homologues. Bulletin 1716 of the American Museum of Natural History 48:533-552. 
1717 Romer AS. 1956. Osteology of the Reptiles. University of Chicago Press, Chicago.

1718 Russell AP, Bauer AM. 2008. The appendicular locomotor apparatus of Sphenodon and normal-

1719 limbed squamates. In: Gans C, Gaunt AS, Adler K, eds. Biology of the Reptilia 24,

1720 Morphology 1. Ithaca, NY: Society for the Study of Amphibians and Reptiles, 1-466.

1721

1722

1723

1724

1725

1726

1727

1728

1729

1730

1731

1732

1733

1734
Schwarz D, Wings O, Meyer CA. 2007. Super sizing the giants: first cartilage preservation at a sauropod dinosaur limb joint. Journal of the Geological Society, London 164:61-65.

Schwarz D, Frey E, Meyer CA. 2007. Novel Reconstruction of the Orientation of the Pectoral Girdle in Sauropods. The Anatomical Record 290:32-47.

Senter P. 2005. Function in the stunted forelimbs of Mononykus olecranus (Theropoda), a dinosaurian anteater. Paleobiology 31:373-381.

Senter $P$, Robins JH. 2005. Range of motion in the forelimb of the theropod dinosaur Acrocanthosaurus atokensis, and implications for predatody behaviour. Zoological Journal of London 266:307-318.

Sereno PC. 1993. The pectoral girdle and forelimb of the basal theropod Herrerasaurus ischigualastensis. Journal of Vertebrate Paleontology 13:425-450.

Sereno PC. 2007. Basal Sauropodomorpha: historical and recent phylogenetic hypotheses, with comments on Ammosaurus major (Marsh, 1889). Special Papers in Palaeontology 77:261-289. 
1735 Suzuki D, Hayashi S. 2010. Myology of crocodiles II: Pectoral girdle and forelimb. The

1736 Palaeontological Society of Japan 87:83-102.

1737 Taylor MP, Wedel MJ. 2013. The effect of intervertebral cartilage on neutral posture and range of motion in the necks of sauropod dinosaurs. PLoS ONE 8(10):e78214.

1739

1740

1741

1742

1743

1744

1745

1746

1747

1748

1749

1750

1751

1752

1753
Tsai HP, Holliday CM. 2014. Articular soft tissue anatomy of the archosaur hip joint: structural homology and functional implications. Journal of Morphology 276:601-630.

Upchurch P, Barrett PM, Galton PM. 2007. A phylogenetic analysis of basal sauropodomorph relationships: implications for the origin of sauropod dinosaurs. Special Papers in Palaeontology 77:57-90.

VanBuren CS, Bonnan M. 2013. Forearm posture and mobility in quadrupedal dinosaurs. PLOS ONE 8(9):e74842.

Vanden Berge JC. 1982. Miología de las aves. In: Sisson S, Grossman JD, eds. Anatomía de los animales domésticos. Salvat, 1973-2021.

Vanden Berge JC, Zweers GA. 1993. Myologia. In: Baumel JJ, King AS, Breazile JE, Evans HE, Vanden Berge JC, eds. Handbook of Avian Anatomy: Nomina Anatomica Avium. Massachusetts: Nutall Ornithological Club 23, 189-250.

Vargas-Peixoto D, Da-Rosa AAS, Franca MAG. 2015. Functional and biomechanic aspects of the scapular girdle and forelimbs of Unaysaurus tolentinoi Leal et al., 2004 (Saurischia: Sauropodomorpha). Journal of South American Earth Sciences 61:129-133. 
1754 White MA, Bell PR, Cook AG, Barnes DG, Tischler TR, Bassam BJ, Elliott DA. 2015. Forearm range 1755 of motion in Australovenator wintonensis (Theropoda, Megaraptoridae). PLOS ONE 10(9):e0137709.

1757

1758

1759

1760

1761

1762

1763

1764

1765

1766

1767

1768

1769

1770

Williams SB, Wilson AM, Payne RC. 2007. Functional specialisation of the thoracic limb of the hare (Lepus europeus). Journal of Anatomy 210:491-505.

Williams SB, Wilson AM, Daynes J, Peckham K, Payne RC. 2008. Functional anatomy and muscle moment arms of the thoracic limb of an elite sprinting athlete: the racing greyhound (Canis familiaris). Journal of Anatomy 213:373-382.

Wilson JA. 2002. Sauropod dinosaur phylogeny: critique and cladistic analysis. Zoological Journal of the Linnean Society 136:217-276.

Wilson JA. 2006. Anatomical nomenclature of fossil vertebrates: standardized terms or lingua franca? Journal of Vertebrate Paleontology 26:511-518.

Wilson JA, Sereno PC. 1998. Early evolution and higher-level phylogeny of sauropod dinosaurs. Society of Vertebrate Paleontology Memoir 5:1-68.

Witmer LM. 1995. The extant phylogenetic bracket and the importance of reconstructing soft tissues in fossils. In: Thomason JJ, ed. Functional Morphology in Vertebrate Paleontology. Cambridge: Cambridge University Press, 19-33.

1771 Witton MP. 2015. Were early pterosaurs inept terrestrial locomotors? PeerJ 3:e1018. 
1772 Yates AM. 2007. The first complete skull of the Triassic dinosaur Melanorosaurus Haughton (Sauropodomorpha: Anchisauria). Special Papers in Palaeontology 77:9-55.

1774 Yates AM, Vasconcelos CC. 2005. Furcula-like in the prosauropod dinosaur Massospondylus. Journal of Vertebrate Paleontology 25:466-468.

1776

1777

1778

1779

1780

Yates AM, Bonnan MF, Neveling J, Chinsamy A, Blackbeard MG. 2010. A new transitional sauropodomorph dinosaur from the Early Jurassic of South Africa and the evolution of sauropod feeding and quadrupedalism. Proceedings of the Royal Society of London B 277:787-794. 


\section{Figure 1}

Simplified cladogram of crown group Archosauria depicting the relationships between Crocodylus johnstoni and Mussaurus patagonicus.

Arrows denote stem-based clade definitions. Modified from Brusatte et al., 2010; Otero et al., 2015. 


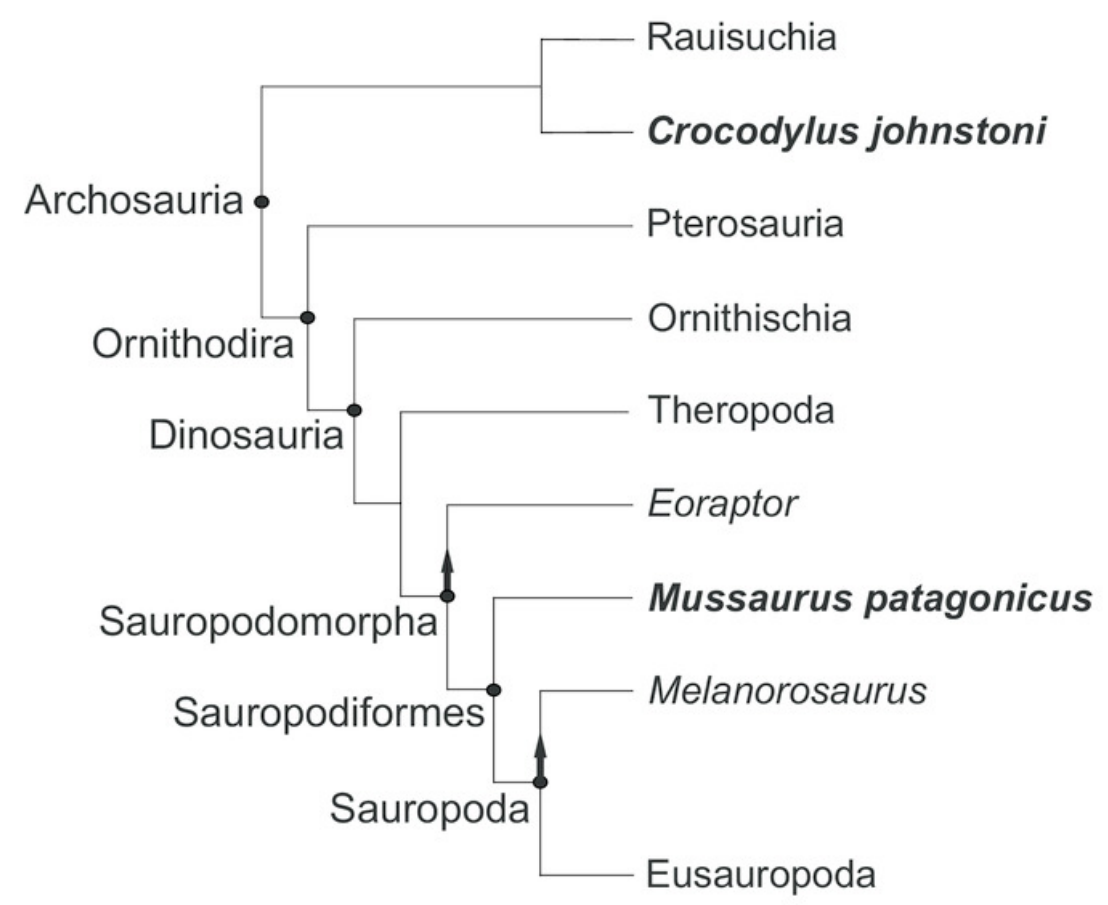




\section{Figure 2}

Crocodylus and Mussaurus models.

Joint axes for rotation $(x, y, z)$ in the reference pose showing the whole forelimb (A-F) and manus (G-I) in cranial (A, B), dorsal (C, D, G), craniolateral $(E, F)$, medial $(H)$ and ventromedial (I) views. A, C, E are Crocodylus and B, D, F-I are Mussaurus. Joint axis ' $\mathrm{x}$ ' (red) corresponds to pronation/supination; ' $y$ ' (green) corresponds to adduction/abduction; and ' $z$ ' (blue) corresponds to extension/flexion, based on the coordinate system described by Baier $\&$ Gatesy (2013).

*Note: Auto Gamma Correction was used for the image. This only affects the reviewing manuscript. See original source image if needed for review. 


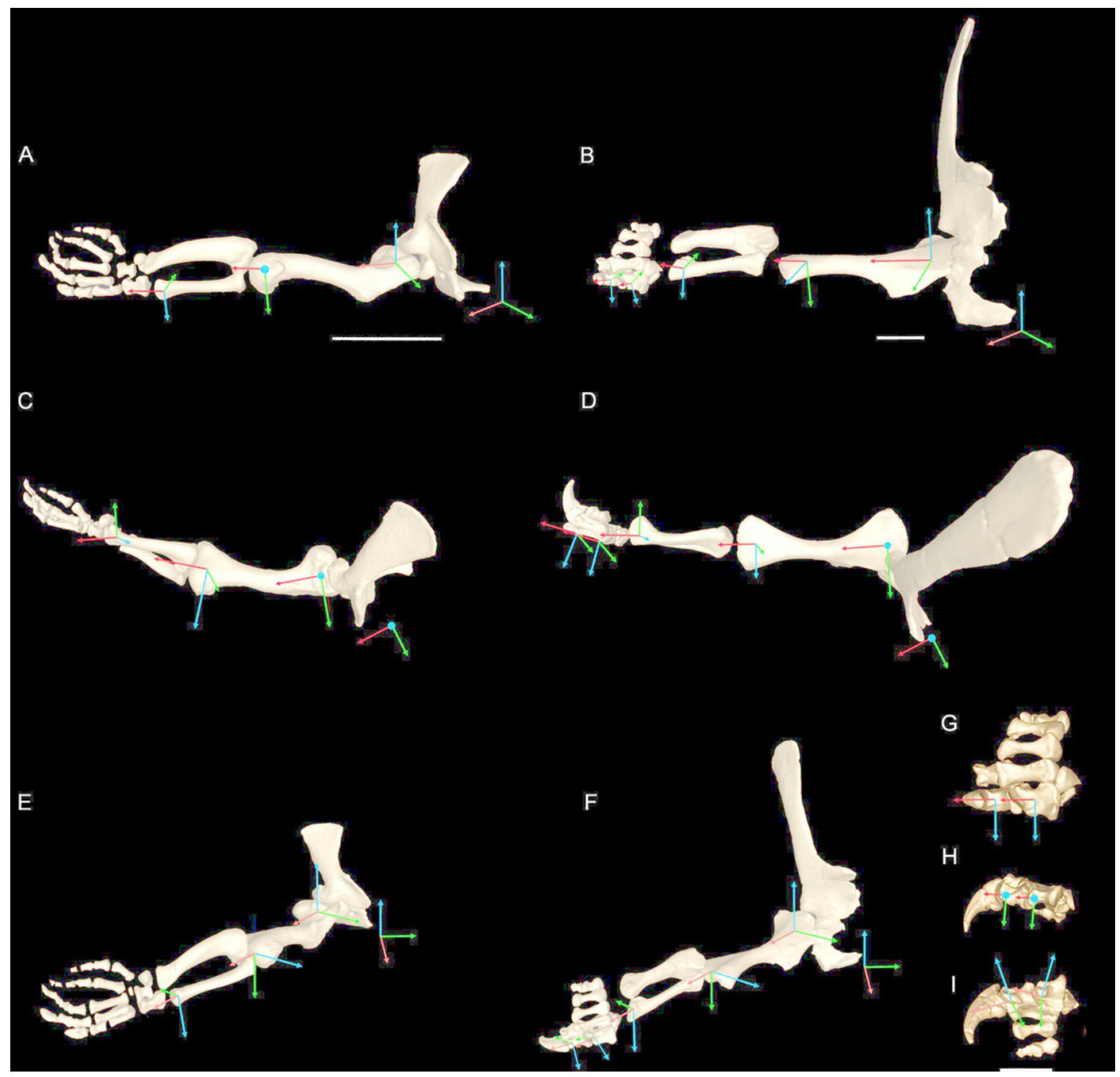




\section{Figure 3}

Muscle reconstruction.

Right forelimb musculoskeletal models for Mussaurus (A-C) and Crocodylus (D-F) models in the resting pose in lateral $(A, D)$, medial $(B, E)$, and dorsomedial $(C, F)$ views. Scale bar: 10 $\mathrm{cm}$.

*Note: Auto Gamma Correction was used for the image. This only affects the reviewing manuscript. See original source image if needed for review.
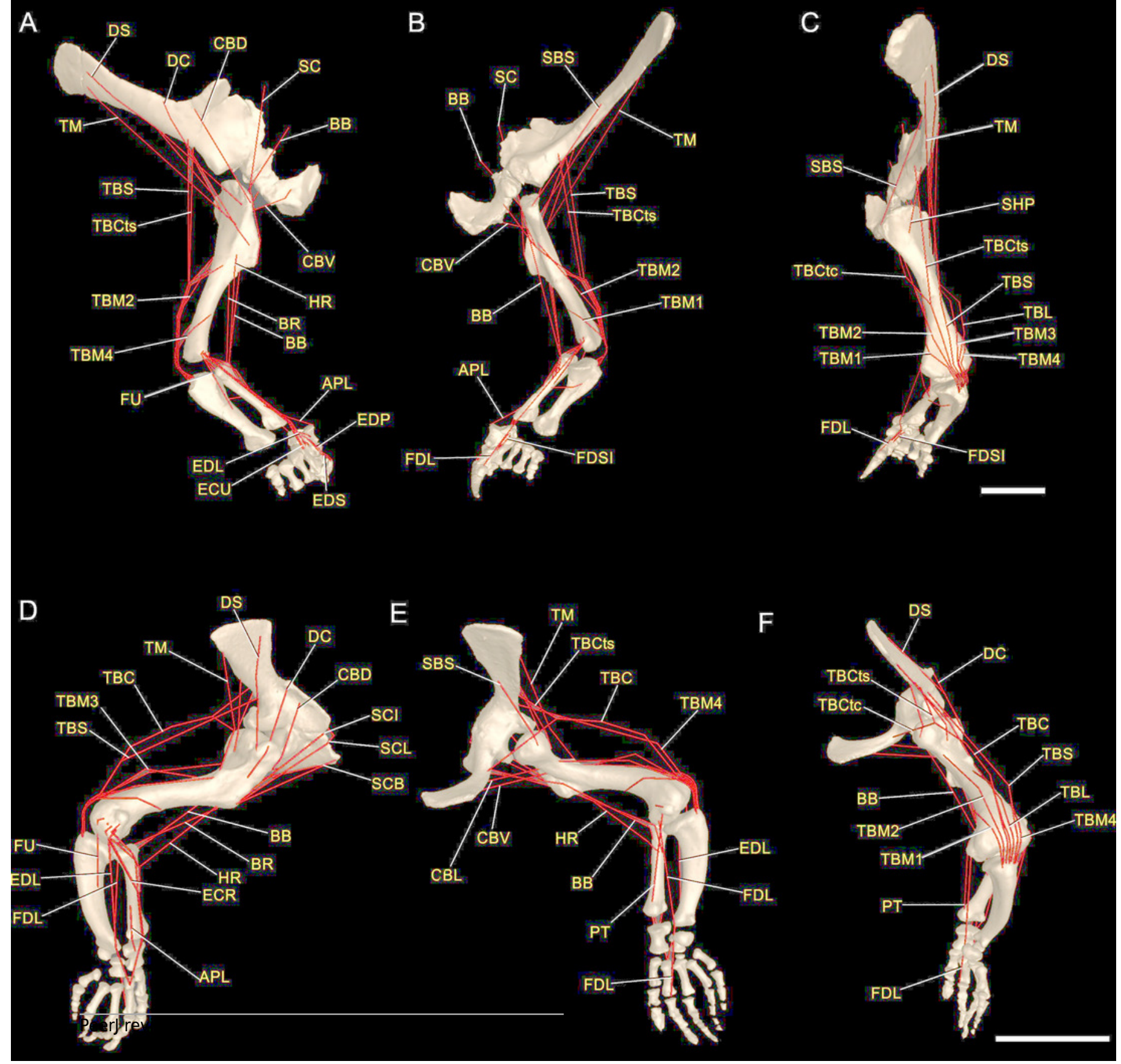


\section{Figure 4}

Joint axis nomenclature for the glenohumeral joint used in this study.

Reference pose in Mussaurus patagonicus showing ' $X$ ' (A, B), ' $Y$ ' (C, D) and ' $Z$ ' (E, F) axes.

Reference pose in Mussaurus showing the vertical scapular blade, with a caudal orientation of the glenohumeral joint, depicting abduction/adduction joint motion (G), and the elevation action of the M. deltoideus scapularis (DS; red line) (H). Resting pose of Mussaurus showing the caudoventrally inclined scapular blade, with a caudoventral orientation of the glenohumeral joint, depicting abduction/adduction joint motion (I), and the retraction action of the M. deltoideus scapularis (DS; red line) (J). Note that the movements/actions depicted in both the reference and resting poses are the same (i.e. they are homologous), but differ in the resulting functions performed, because of the reorientation of the glenohumeral joint via scapular reorientation. 

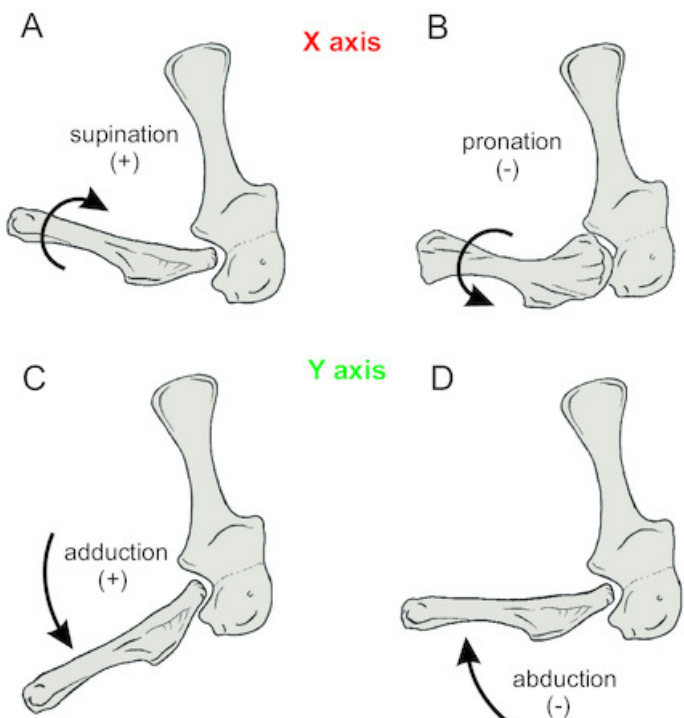

$\mathrm{Y}$ axis
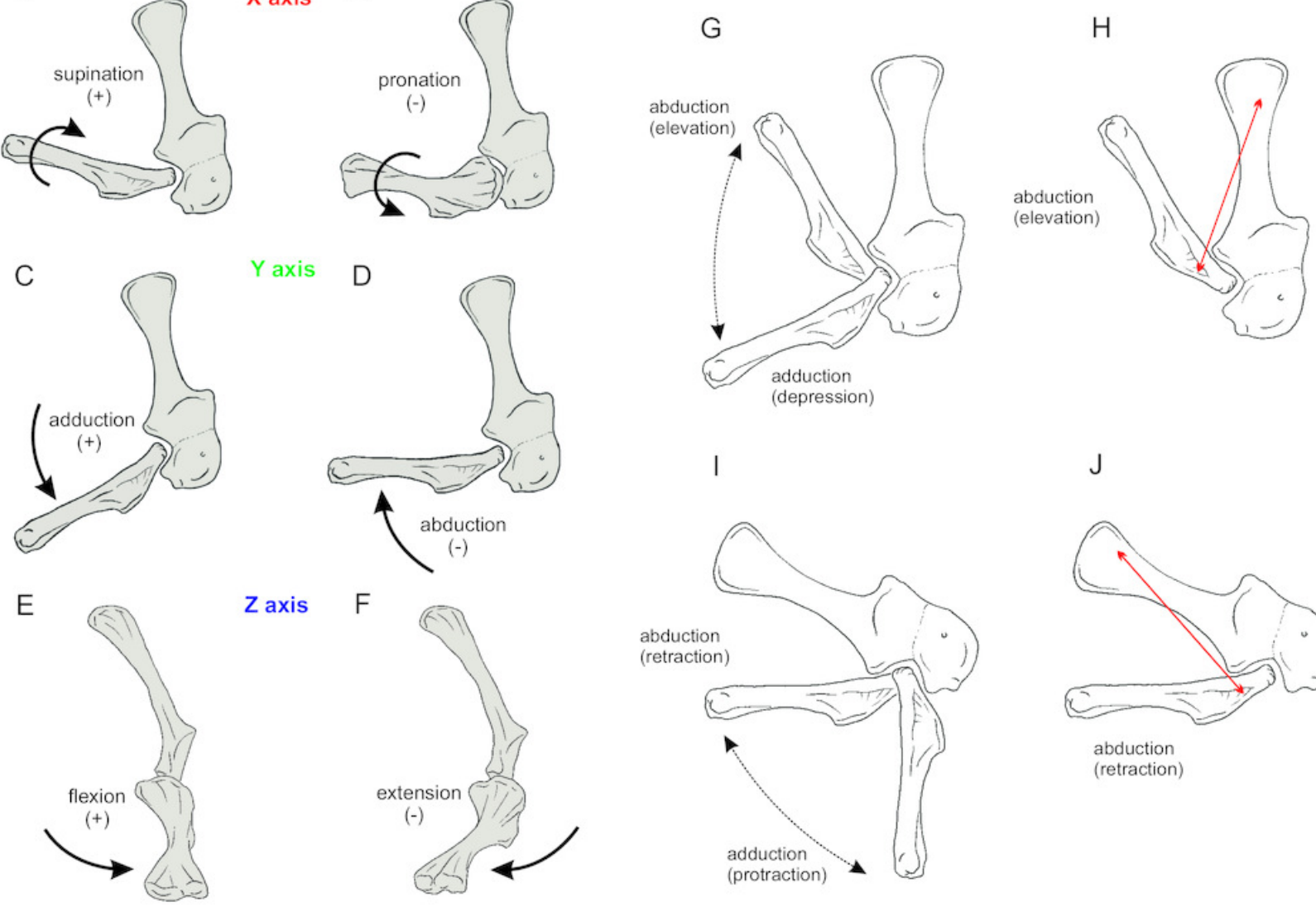

$Z$ axis
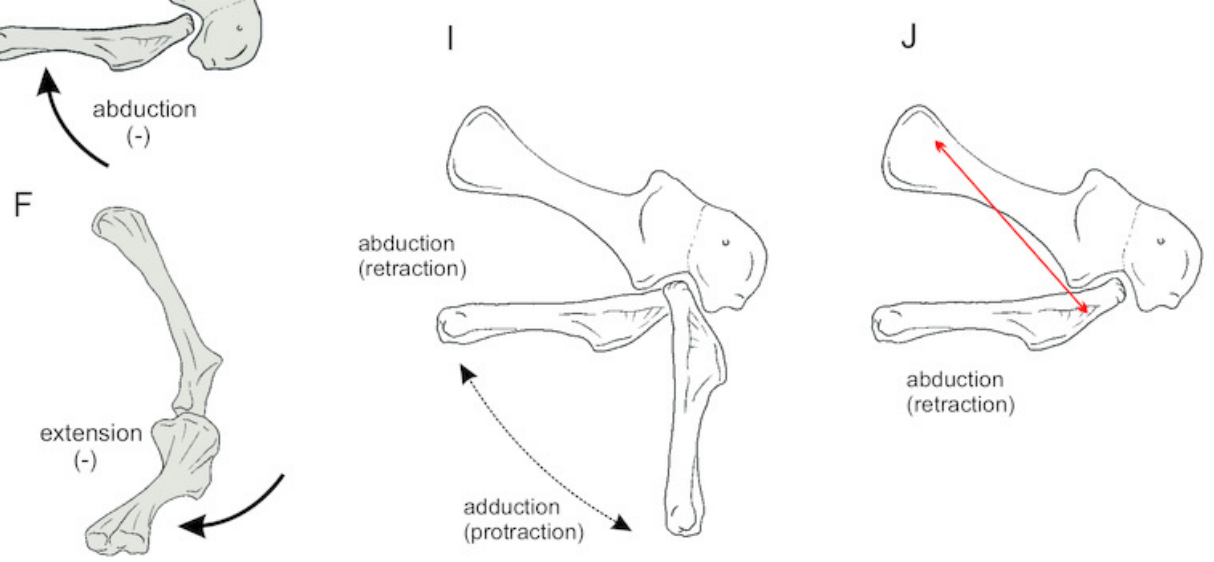


\section{Figure $\mathbf{5}$ (on next page)}

Pronation/supination moment arms around the glenohumeral joint, normalized to humerus segment length, plotted against pronation/supination joint angles for Crocodylus and Mussaurus in the resting pose.
(A) mostly pronators; (B) mostly supinators; (C) mixed pronators/supinators. Negative moment arms and glenohumeral angles correspond to pronation, while positive values correspond to supination. For muscle abbreviations see Table 1. For a similar plot using glenohumeral extension/flexion joint angle on the x-axis, see Fig. S8. 

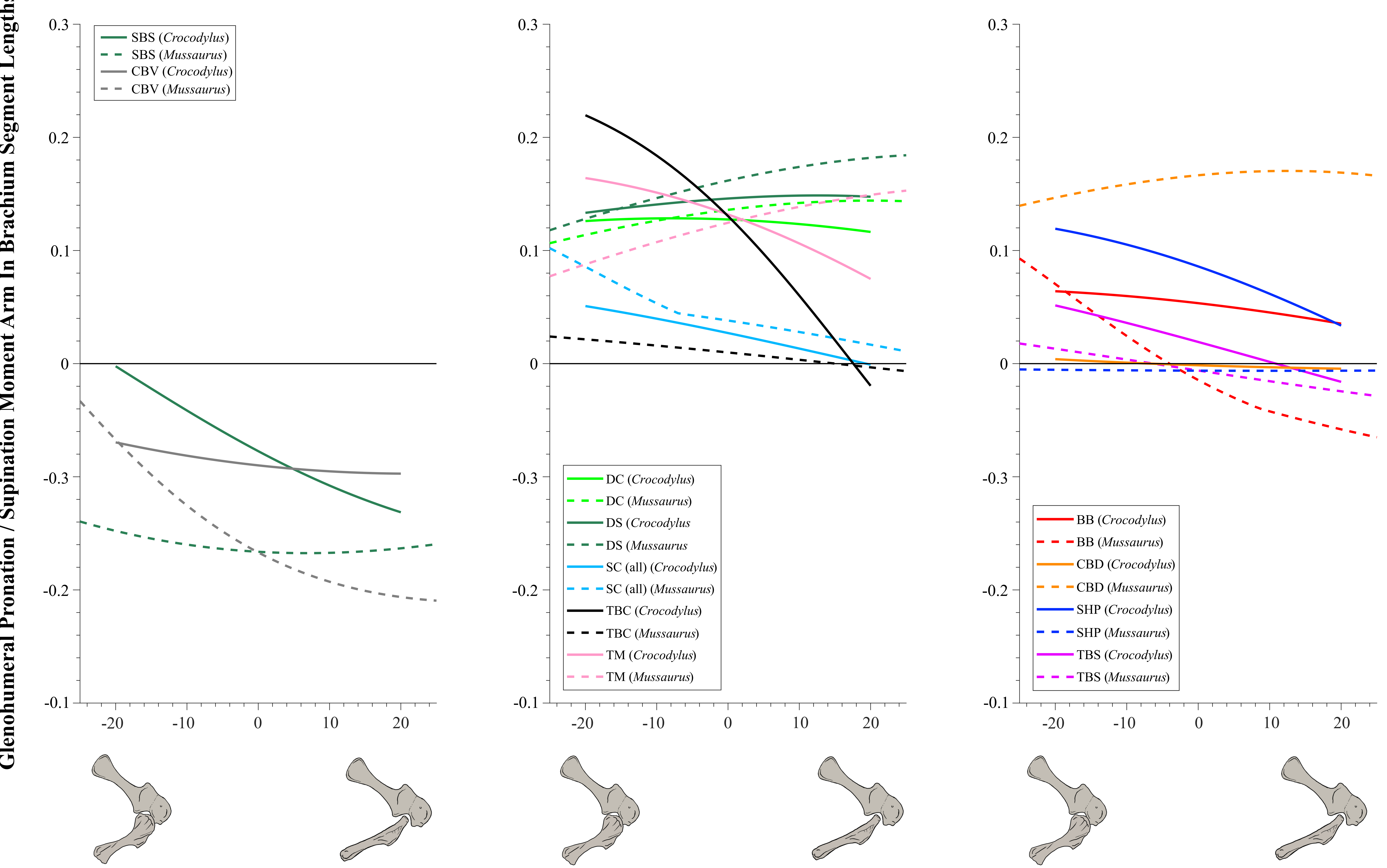
Figure $\mathbf{6}$ (on next page)

Extension/flexion moment arms around the glenohumeral joint, normalized to humerus segment length, plotted against extension/flexion joint angles for Crocodylus and Mussaurus in the resting pose.

(A) mostly extensors; (B) mostly flexors; (C) mixed extensors/flexors. Negative moment arms and glenohumeral angles correspond to extension, while positive values correspond to flexion. For muscle abbreviations see Table 1. 

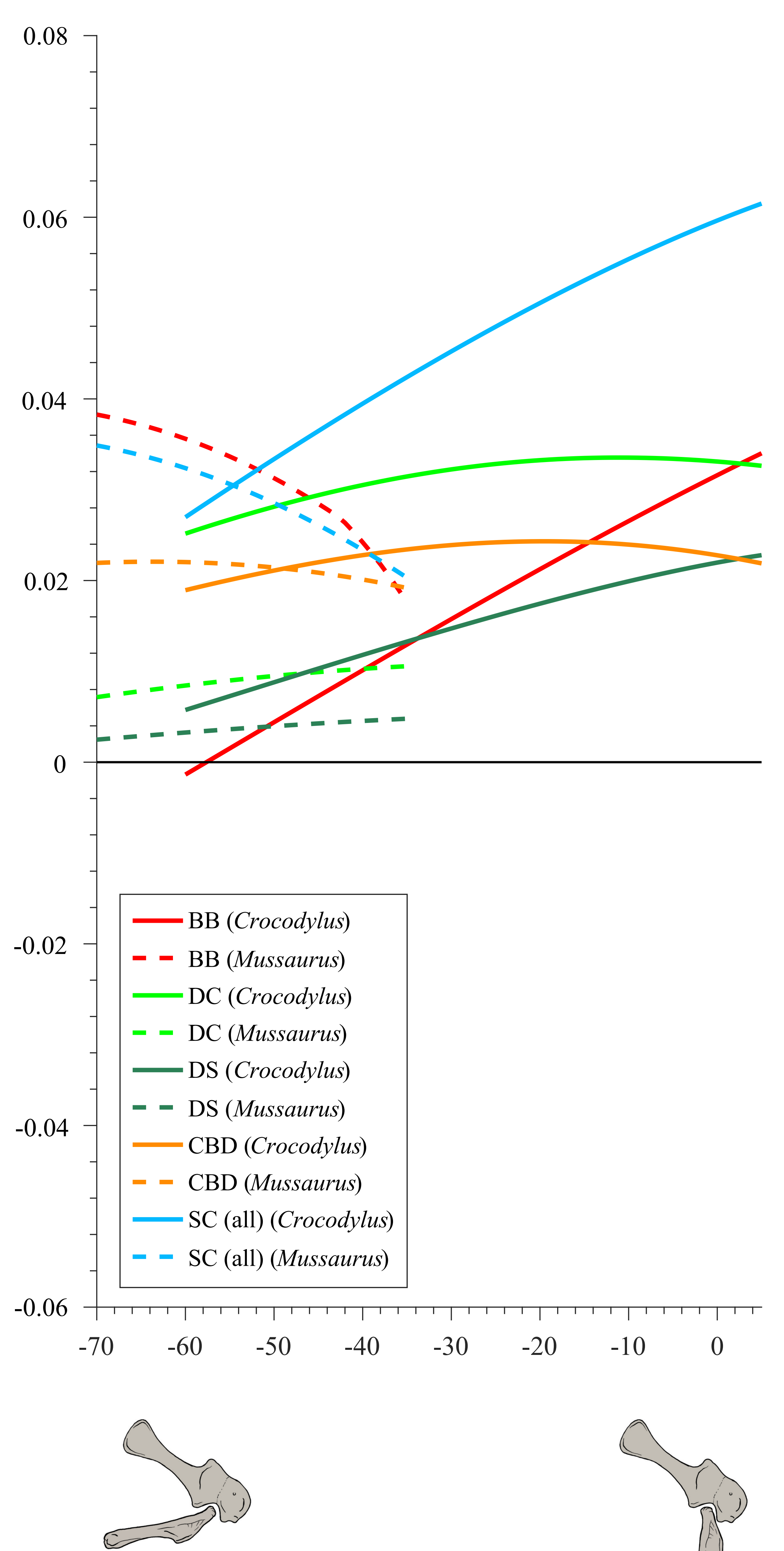

- - SBS (Mussaurus) — SHP (Crocodylus) - - SHP (Mussaurus) _ TM (Crocodylus) - - TM (Mussaurus) - - TBC $(M u s$

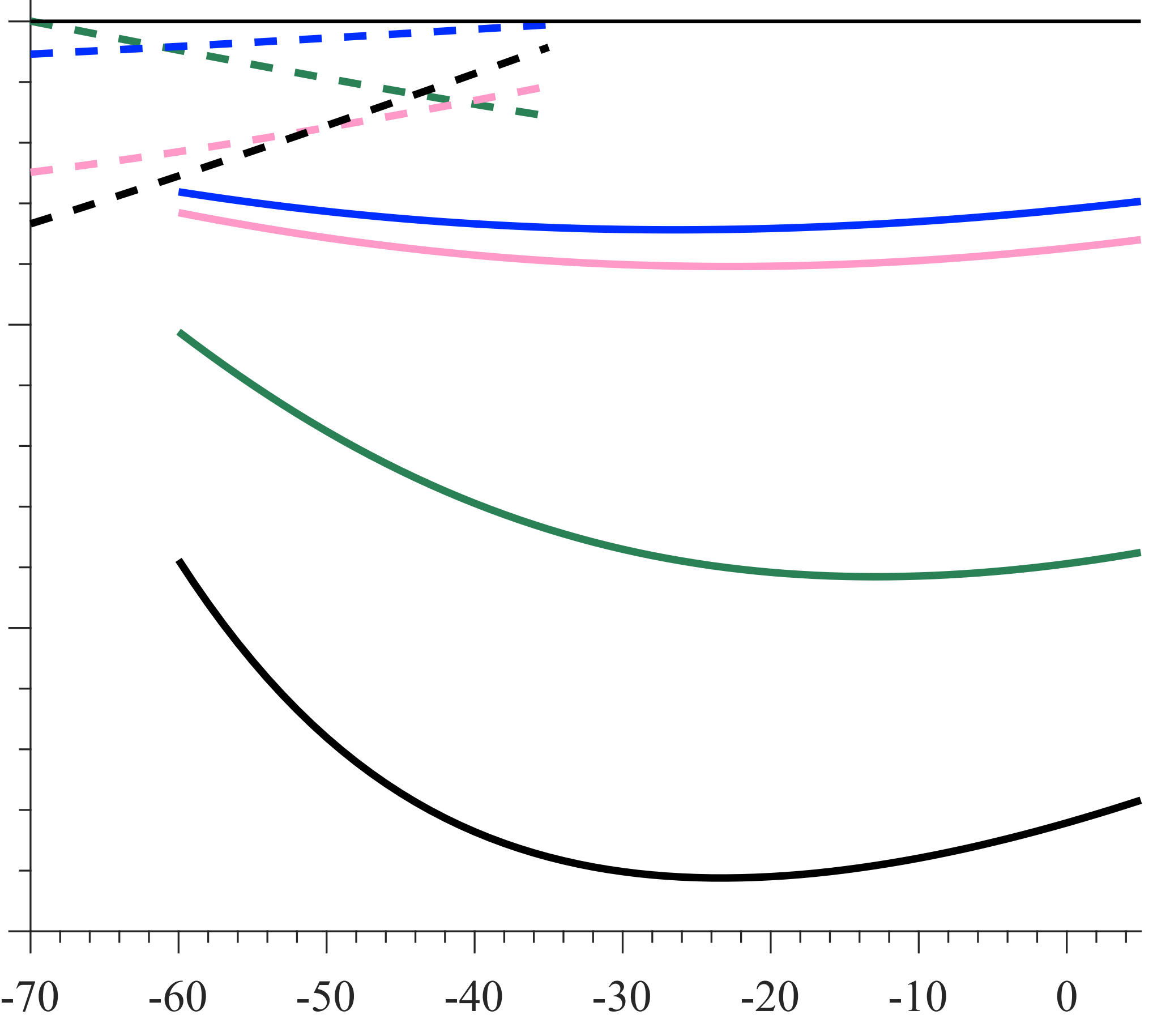

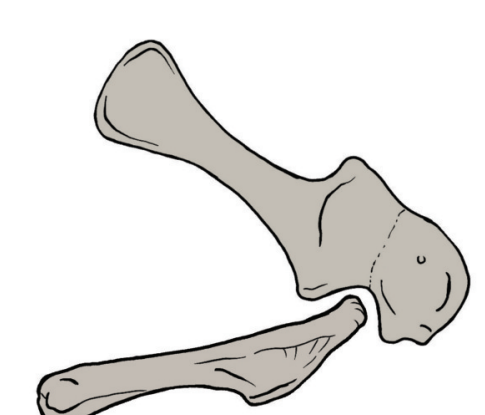
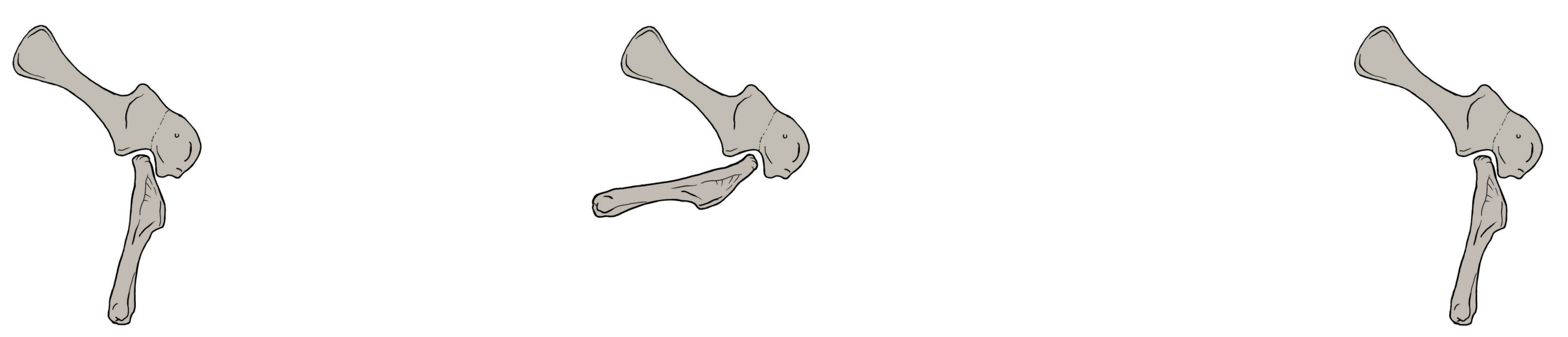

0.08

Mixed Extensors / Flexors

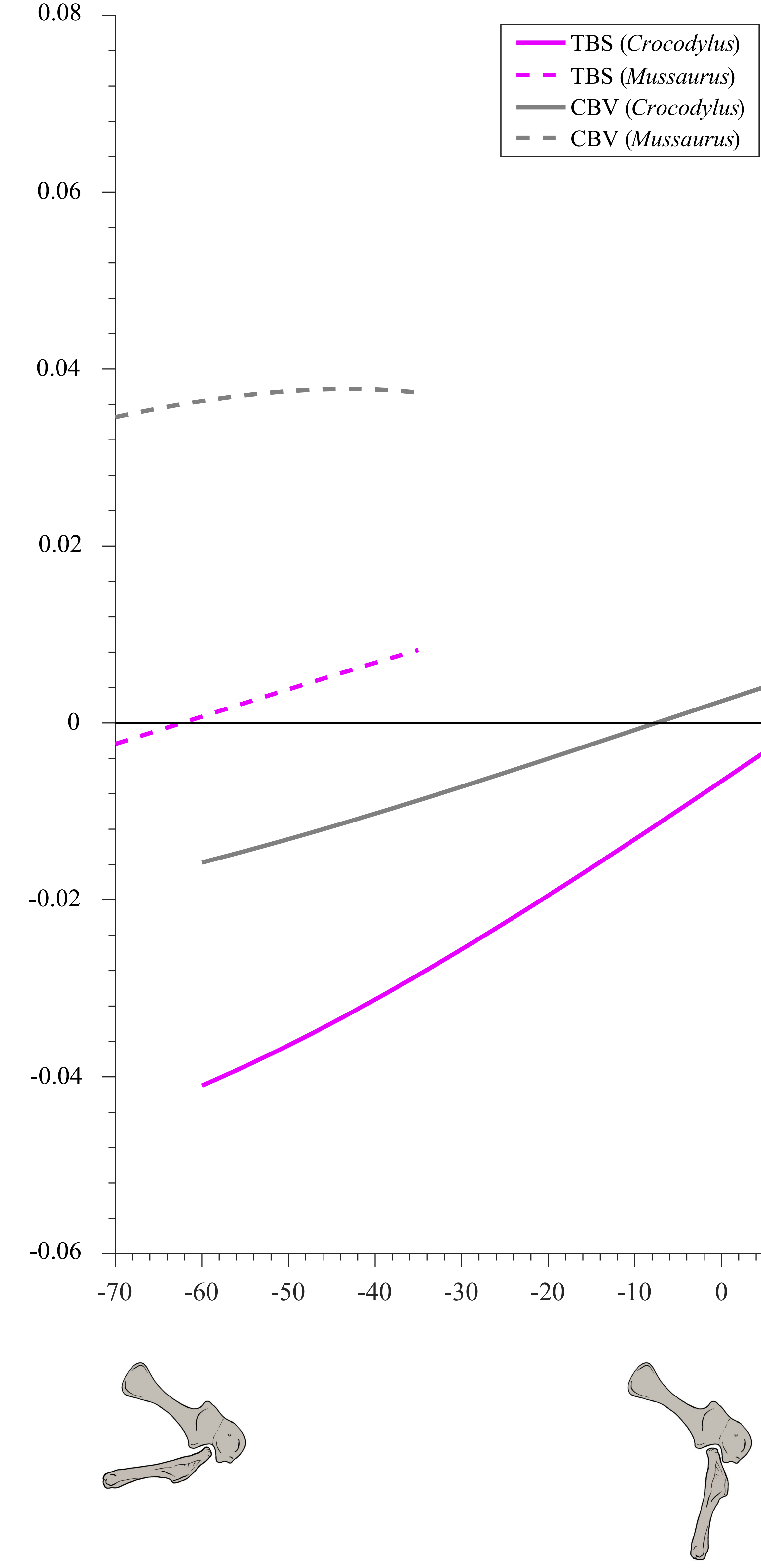


Figure 7 (on next page)

Abduction/adduction moment arms around the glenohumeral joint, normalized to humerus segment length, plotted against abduction/adduction joint angles for Crocodylus and Mussaurus in the resting pose.

(A) mostly abductors; (B) mostly adductors; (C) mixed abductors/adductors. Negative moment arms and glenohumeral angles correspond to abduction, while positive values correspond to adduction. For muscle abbreviations see Table 1. For a similar plot using glenohumeral extension/flexion joint angle on the x-axis, see Fig. S9. 

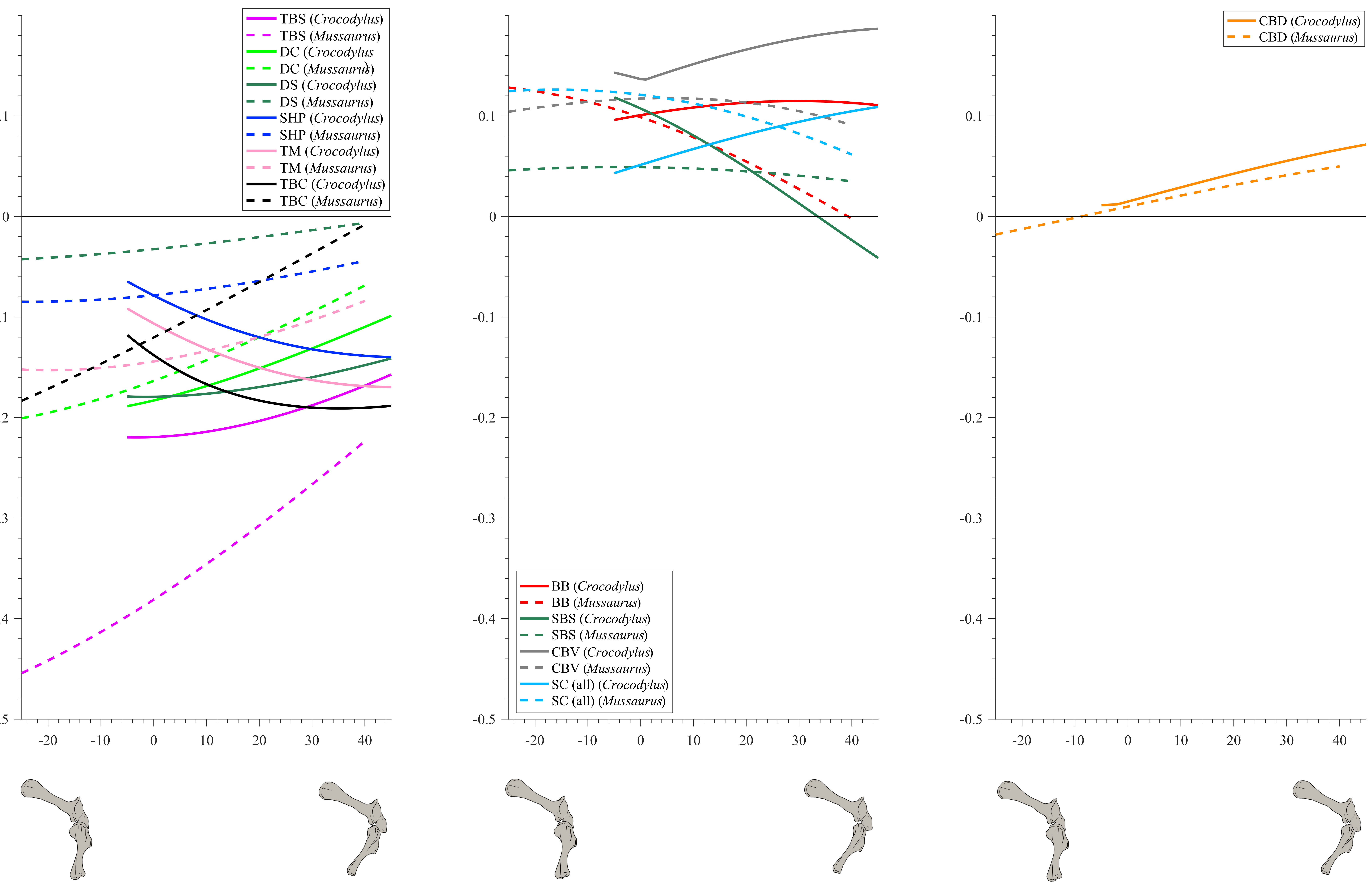

Peer) reviewing PDF | (2017:04:17707:1:1:CHECK 3 Oct 2017) 
Figure 8 (on next page)

Extension/flexion moment arms around the elbow joint, normalized to radius-ulna segment length, plotted against extension/flexion joint angles for Crocodylus and Mussaurus in the resting pose.

(A) mostly extensors; (B) mostly flexors; (C) mixed extensors/flexors. Negative moment arms correspond to extension, while positive values correspond to flexion. Zero elbow angle corresponds to full extension, while larger angles correspond to flexion. For muscle abbreviations see Table 1. 


\section{Figure 9 (on next page)}

Extension/flexion moment arms around the wrist, normalized to metacarpal II length, plotted against extension/flexion joint angles for Crocodylus and Mussaurus.

(A) mostly extensors; (B) mostly flexors. Negative moment arms and wrist joint angles correspond to extension, while positive values correspond to flexion. For muscle abbreviations see Table 1. 


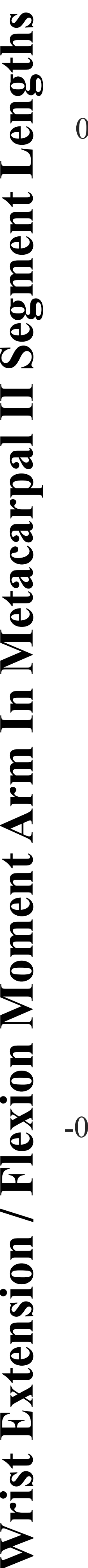

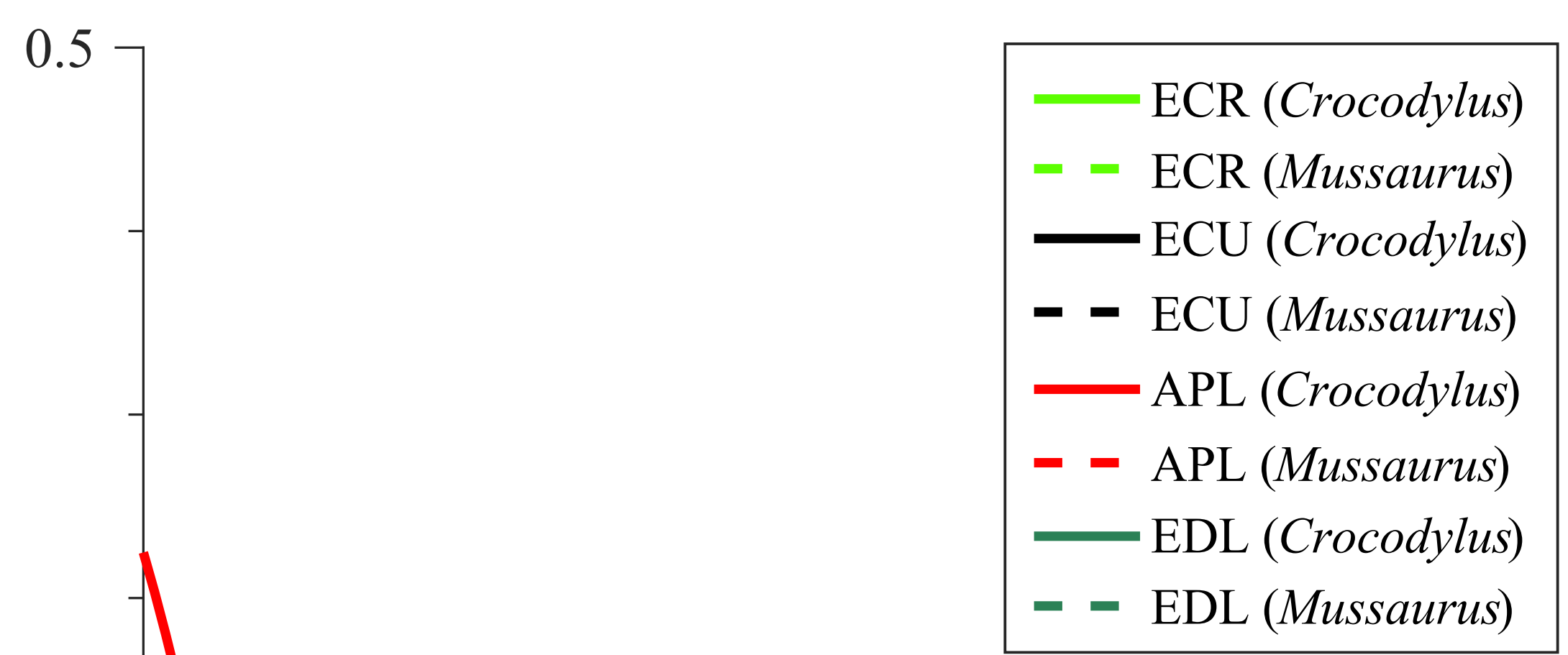

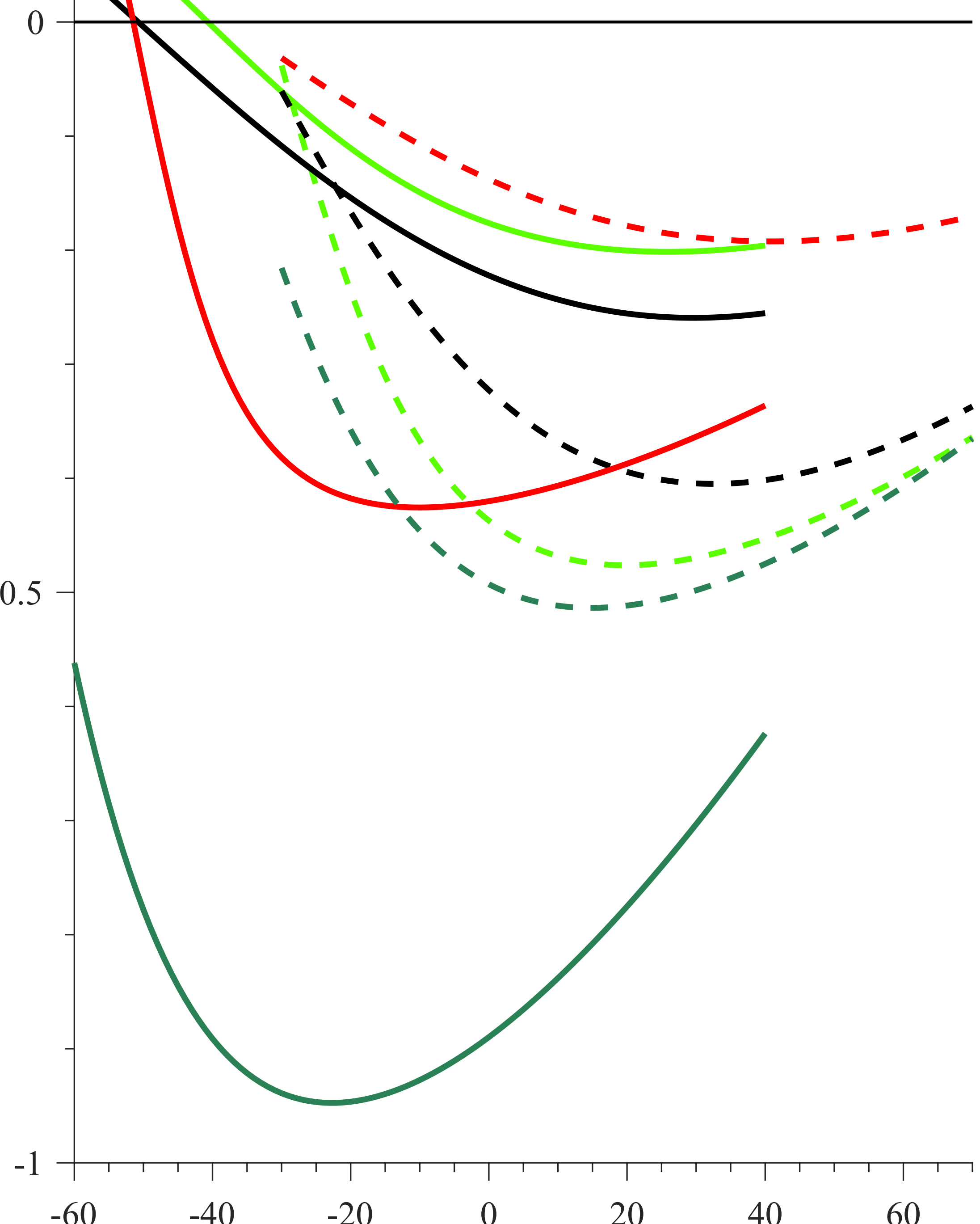
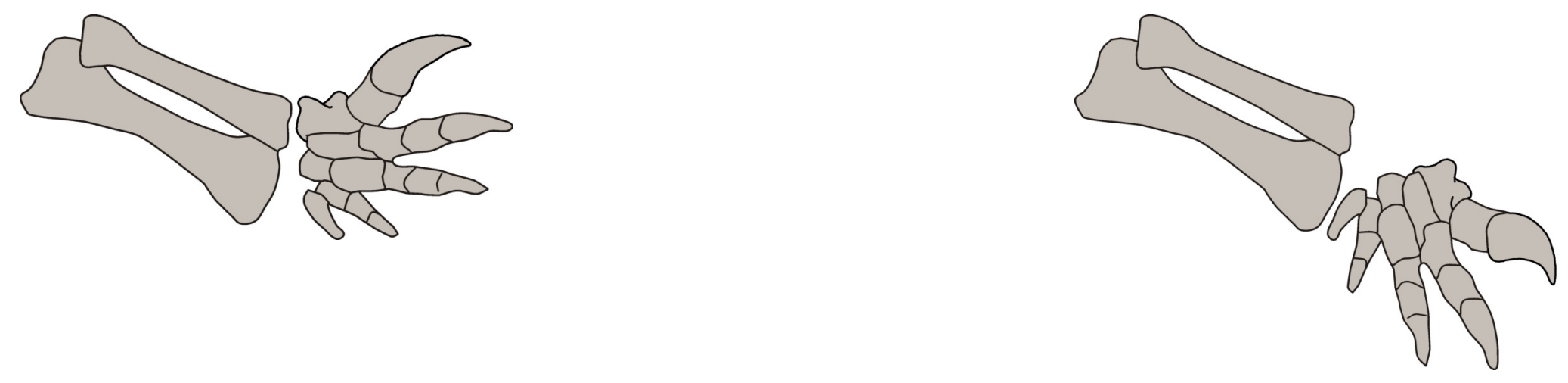

B

Manuscript Mostlye Flexers
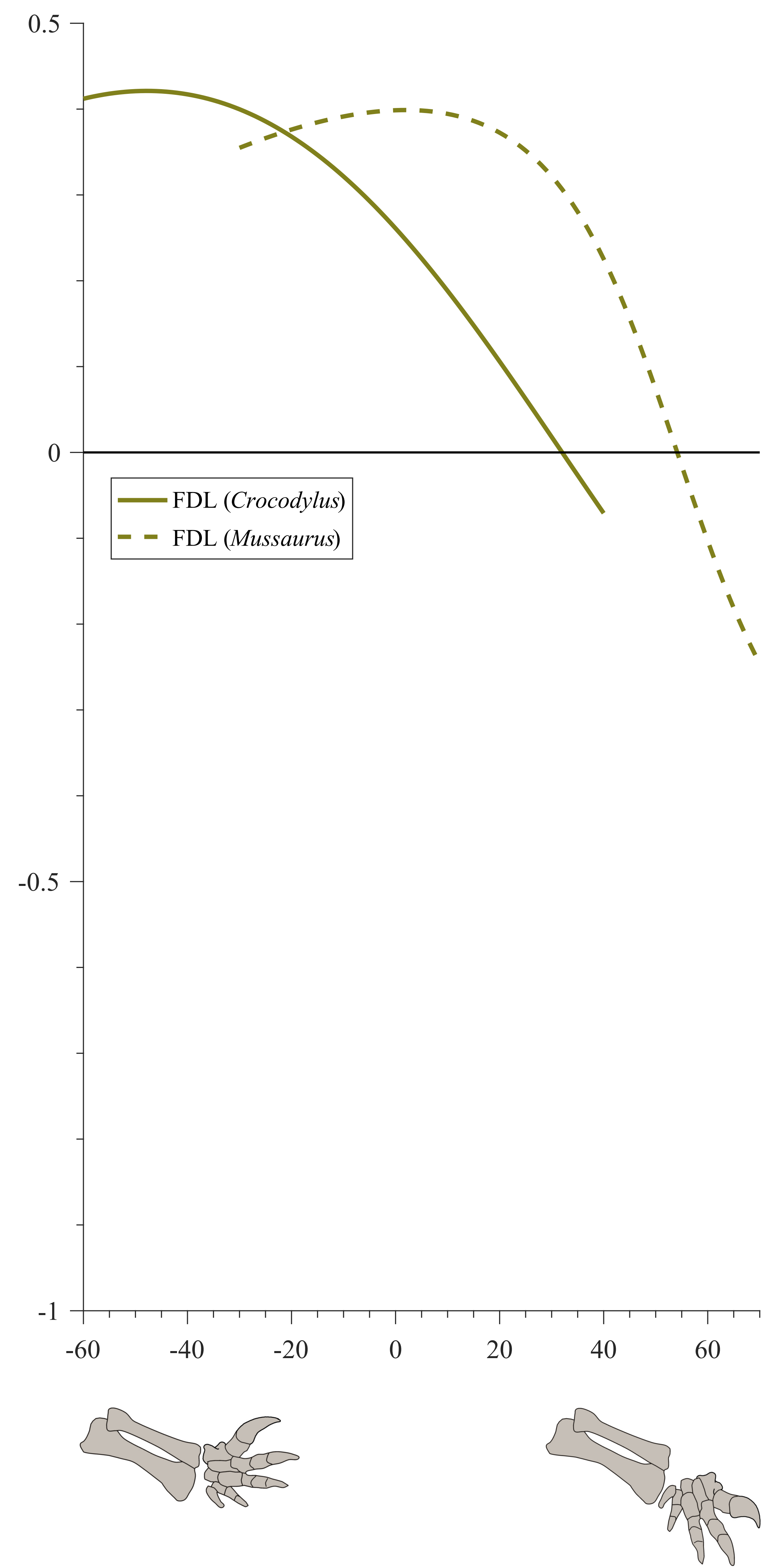
Figure 10 (on next page)

Extension/flexion moment arms (not normalized) around the metacarpo-phalangeal joints (MCP), plotted against extension/flexion joint angles for Mussaurus.

(A) mostly extensors; (B) mostly flexors. Negative moment arms and joint angles correspond to extension, while positive values correspond to flexion. For muscle abbreviations see Table 1. 


\section{PeeMbstly Extensors}
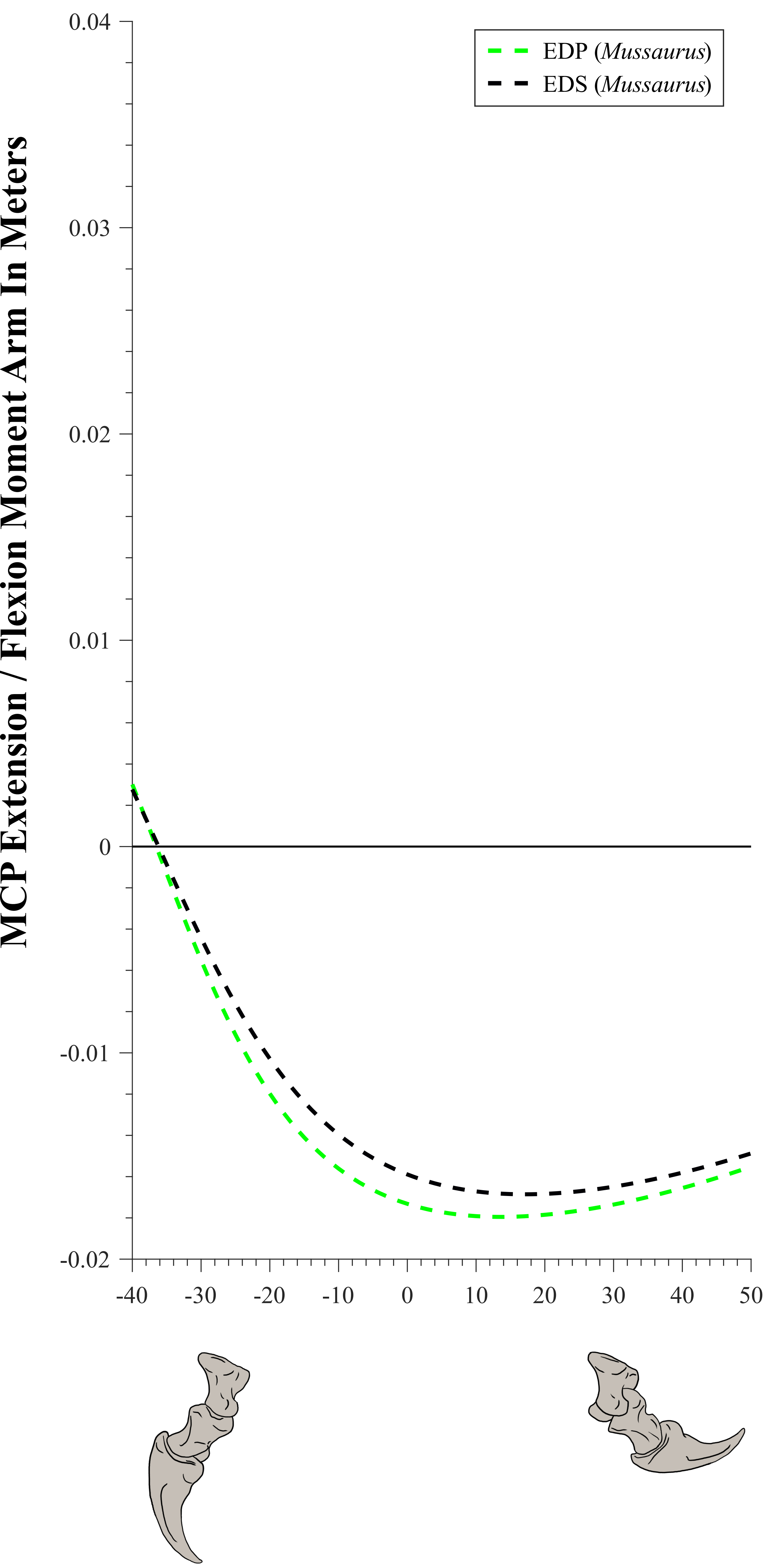

Manuscript MostlyeElexers

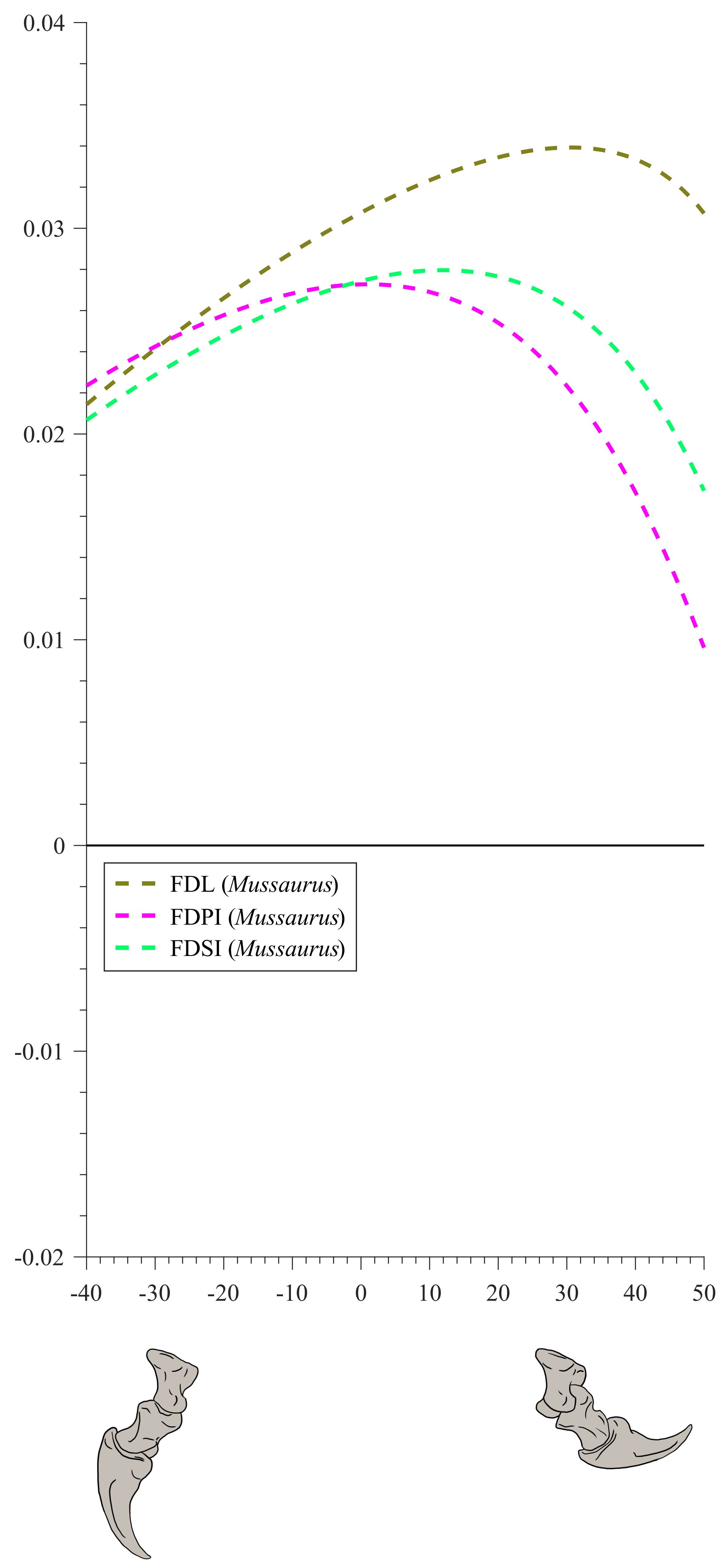


Figure 11 (on next page)

Extension/flexion moment arms (not normalized) around the interphalangeal joints (INP), plotted against flexion/extension joint angles for Mussaurus.

(A) extensors; (B) flexors. Negative moment arms and joint angles correspond to extension, while positive values correspond to flexion. For muscle abbreviations see Table 1. 
A Peelastly Extensors
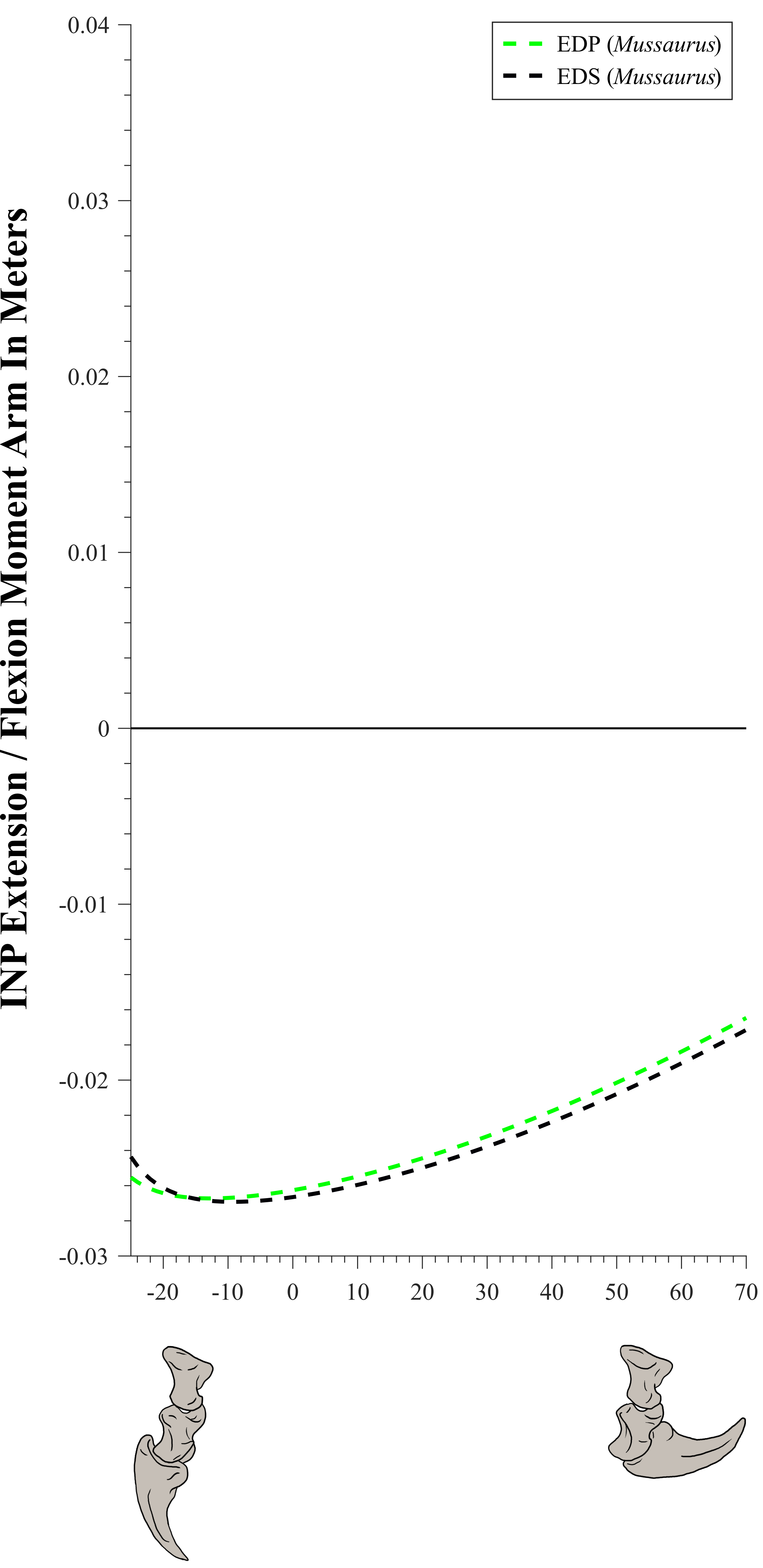

Manuscript tohostivdatexors

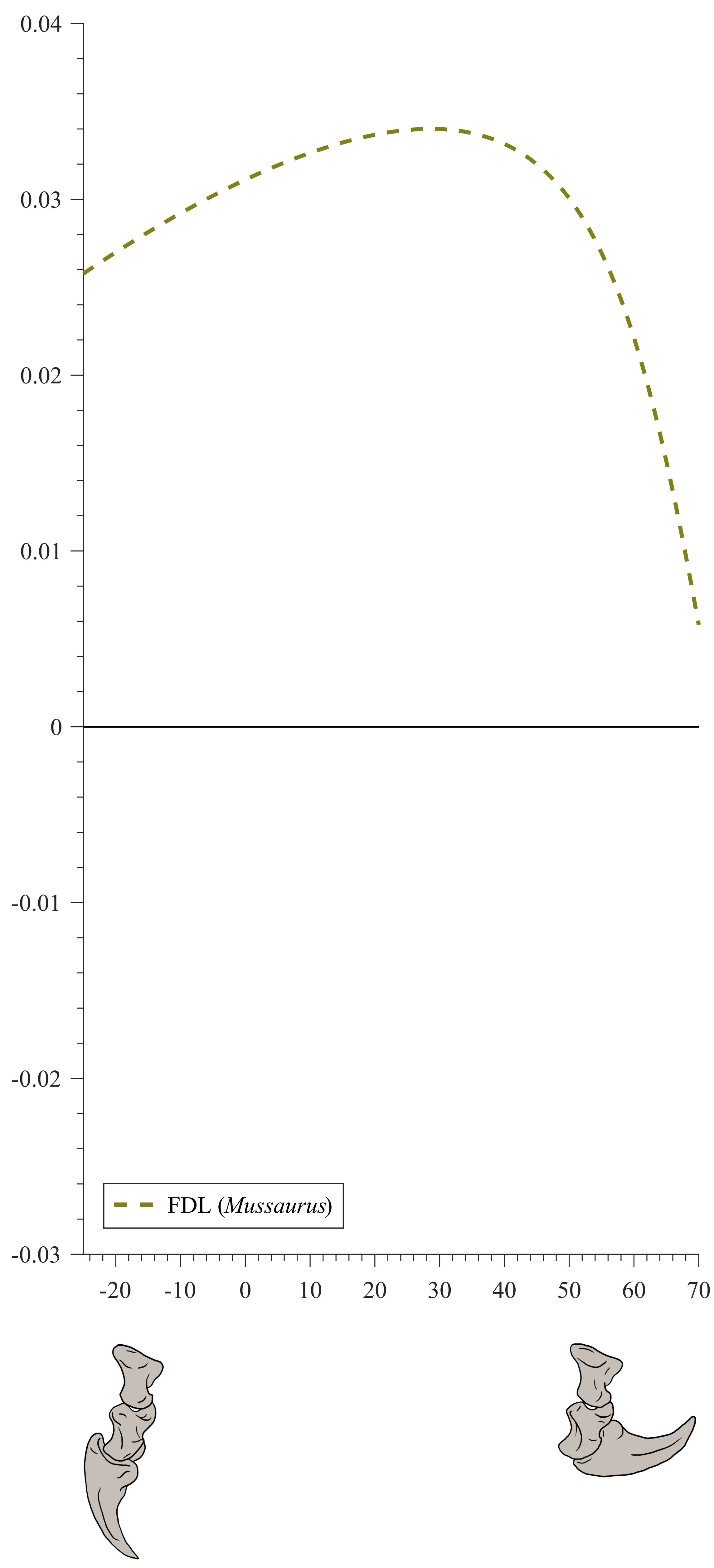




\section{Figure 12}

Terminology for homologous joint movements as the glenohumeral articular surface transformed across Archosauria.

(A) Crocodylus and (B) a generalized bird showing homologous joint movement (in this case perpendicular to the long axis of the glenoid) along the extremes of locomotor patterns within Archosauria. (C) Evolution of muscle action around the flexion/extension axis along the ornithodiran line from the ancestral archosaurian pattern for a homologous movement. Same colour/tone indicates the same glenohumeral joint orientation. Line drawings modified from: Crocodylus and generalized bird (Gatesy \& Baier, 2005); Pterosauria (Witton, 2015); Ornithischia (Maidment \& Barrett, 2011); Camarasaurus (Wilson \& Sereno, 1998) and Tawa (Burch, 2014). Abbreviations: ad., adduction; fl., flexion. 
A

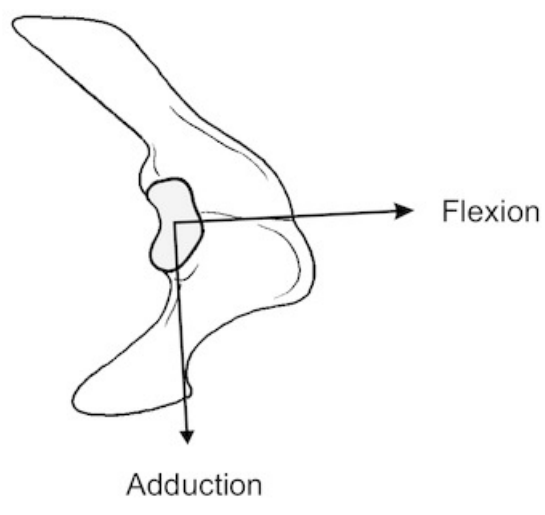

Crocodylus
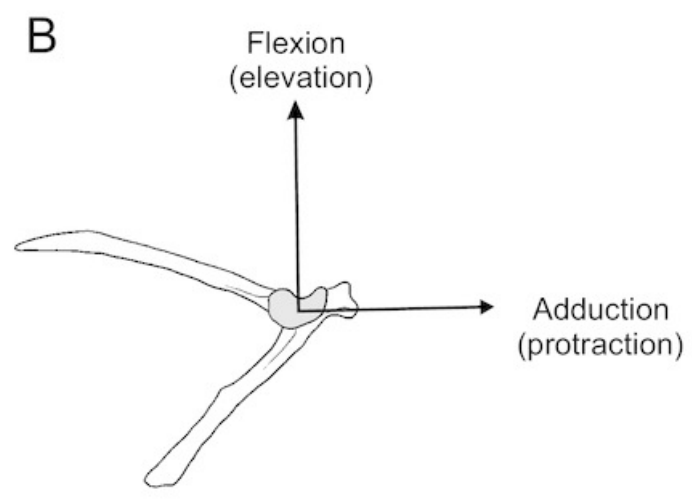

Birds

C

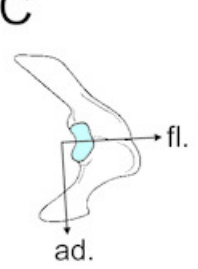

Crocodylus
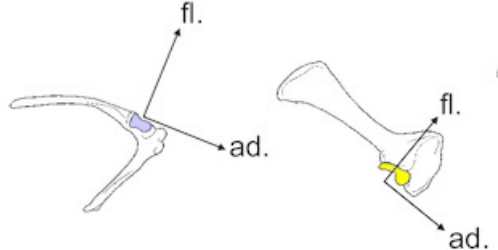

Ornithischia
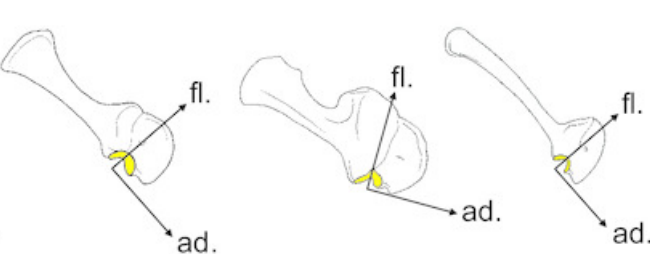

fl.

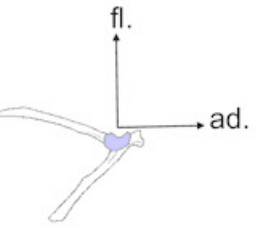

Mussaurus

Camarasaurus

Tawa

Birds
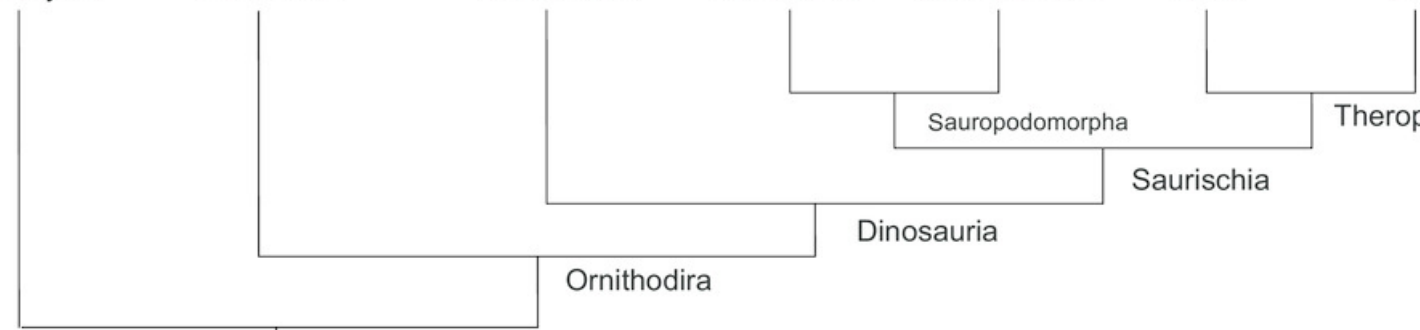

Sauropodomorpha

Theropoda

Saurischia

Archosauria 


\section{Figure 13}

Sensitivity analysis of elbow extensor muscles in Mussaurus patagonicus (right elbow in lateral view; resting pose).

Cartilage diameter is shown for (A) reduced by $25 \%$ from original, (B) original and (C) enlarged by $25 \%$ from original.

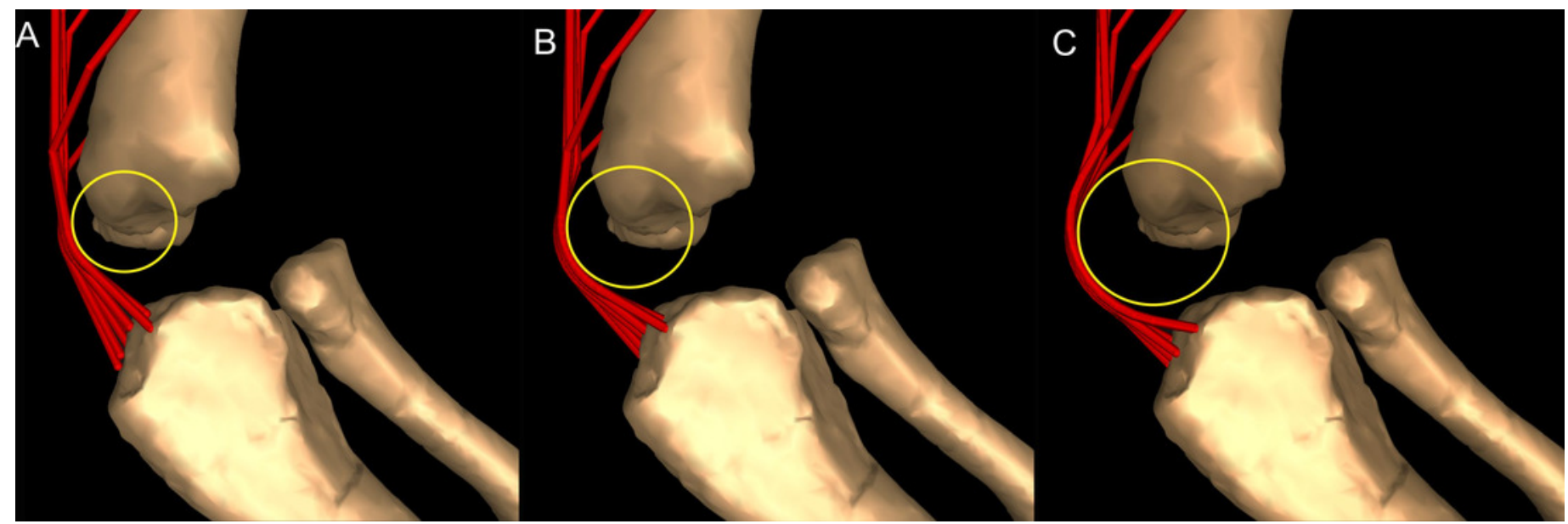


Figure 14(on next page)

Sensitivity analysis of elbow extensor muscles in Mussaurus patagonicus.

Moment arms around the elbow joint (not normalized), plotted against extension/flexion joint angles for Mussaurus in the resting pose; for various elbow cartilage assumptions (see Fig. 13 and Methods). Negative moment arms correspond to extension. Zero elbow angle corresponds to full extension, while larger angles correspond to flexion. For muscle abbreviations see Table 1. 



\section{Figure 15}

Antebrachial bones of Mussaurus patagonicus.

Radius and ulna (A) showing the articulation of the distal ends in medial (B), distomedial (C), dorsolateral (D) and lateral (E) views. Not to scale.

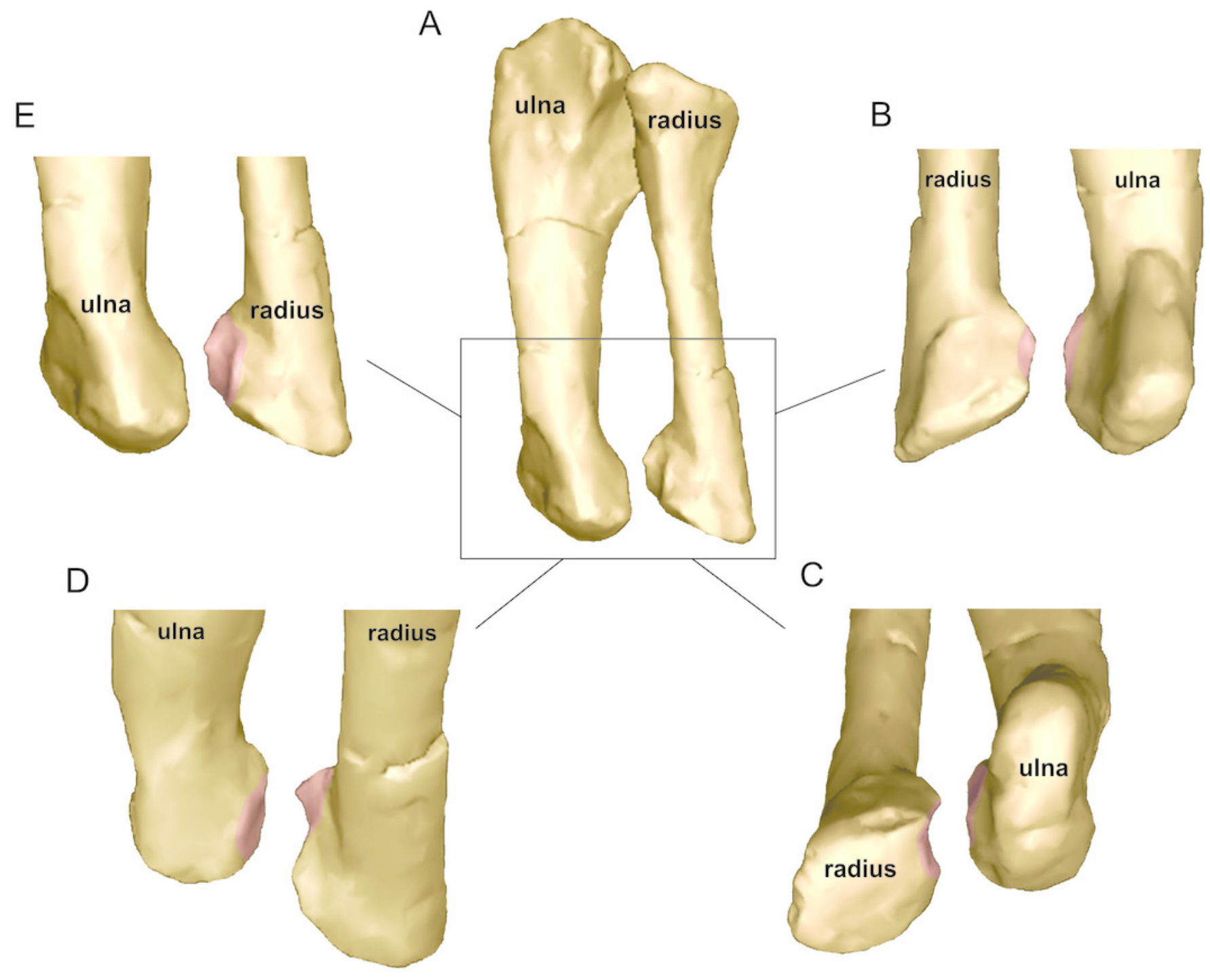




\section{Figure 16}

Antebrachial articulations of Mussaurus patagonicus.

Non-pronated (i.e. semi-pronated, sensu Hutson \& Hutson, 2015, 2017) (A, C, E) and semipronated (i.e. $30^{\circ}$ of medial rotation/pronation from $\left.A\right)(B, D, F)$ poses depicting the relationships among antebrachial bones. Radius and ulna in proximal $(A, B)$ views, forelimb in cranial (C, D) views, and manus in proximal $(E, F)$ views. 


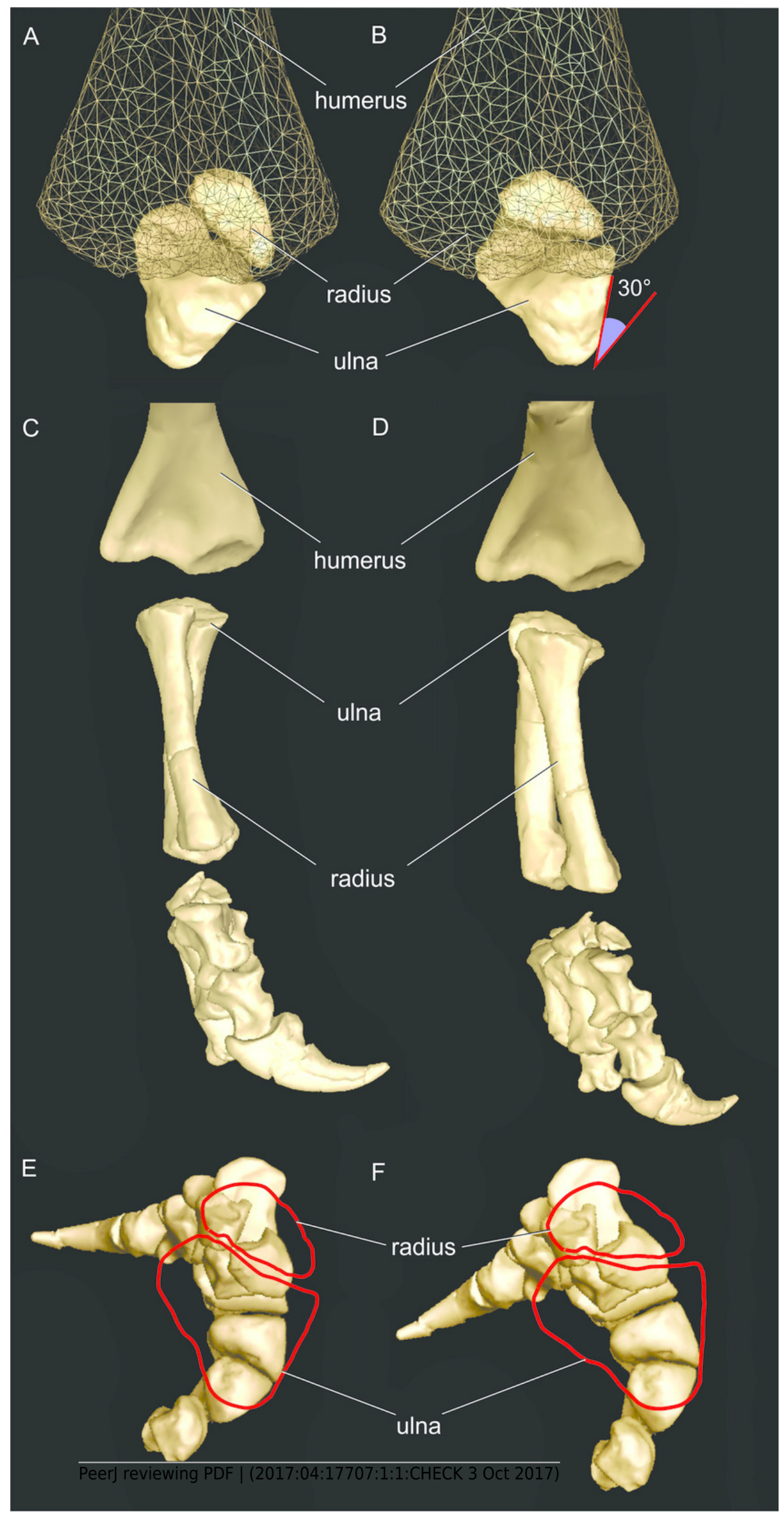




\section{Table $\mathbf{1}$ (on next page)}

Shoulder and forelimb muscles inferred to be present in Mussaurus patagonicus, and their approximate locations. 
1 Table 1. Shoulder and forelimb muscles inferred to be present in Mussaurus patagonicus, and their

2 approximate locations.

\begin{tabular}{|c|c|c|c|c|c|}
\hline Muscle & Abbreviation & Origin & $\begin{array}{l}\text { Level of } \\
\text { Inference }\end{array}$ & Insertion & $\begin{array}{l}\text { Level of } \\
\text { Inference }\end{array}$ \\
\hline Deltoideus scapularis & DS & $\begin{array}{l}\text { Lateral surface of the } \\
\text { scapular blade }\end{array}$ & $\mathrm{II}^{\prime}$ & $\begin{array}{l}\text { Caudal side of the humerus, } \\
\text { close to the humeral head }\end{array}$ & II \\
\hline Deltoideus clavicularis & DC & $\begin{array}{l}\text { Acromial region along } \\
\text { the craniodorsal } \\
\text { surface of the } \\
\text { scapula }\end{array}$ & $\mathrm{I}^{\prime}$ & $\begin{array}{l}\text { Caudal surface of the } \\
\text { deltopectoral crest }\end{array}$ & $l^{\prime}$ \\
\hline Teres major & TM & $\begin{array}{l}\text { Caudolateral surface } \\
\text { of the scapular } \\
\text { blade, on the distal } \\
\text { half of the blade }\end{array}$ & $I^{\prime}$ & $\begin{array}{l}\text { Caudal surface of the } \\
\text { humerus, medial to the } \\
\text { deltopectoral crest }\end{array}$ & II \\
\hline Subscapularis & SBS & $\begin{array}{l}\text { Medial surface of the } \\
\text { scapular blade, just } \\
\text { above the } \\
\text { ventromedial ridge }\end{array}$ & $\mathrm{I}^{\prime}$ & $\begin{array}{l}\text { Proximal end of the } \\
\text { humerus, medial to the } \\
\text { humeral head }\end{array}$ & I \\
\hline $\begin{array}{l}\text { Scapulohumeralis } \\
\text { posterior }\end{array}$ & SHP & $\begin{array}{l}\text { Caudal margin of the } \\
\text { scapular blade, } \\
\text { above the scapular } \\
\text { glenoid lip }\end{array}$ & $\mathrm{I}^{\prime}$ & $\begin{array}{l}\text { Proximocaudal surface of } \\
\text { the humerus, below the } \\
\text { humeral head }\end{array}$ & $\mathrm{I}^{\prime}$ \\
\hline $\begin{array}{l}\text { Supracoracoideus } \\
\text { complex }\end{array}$ & $\mathrm{SC}$ & & & & \\
\hline S. longus & $\mathrm{SCL}$ & $\begin{array}{l}\text { Medial scapula- } \\
\text { coracoid boundary }\end{array}$ & $I^{\prime}$ & $\begin{array}{l}\text { Distal portion of the } \\
\text { deltopectoral crest }\end{array}$ & I \\
\hline S. intermedius & $\mathrm{SCl}$ & $\begin{array}{l}\text { Lateral scapula- } \\
\text { coracoid boundary }\end{array}$ & $\mathrm{I}^{\prime}$ or II' & $\begin{array}{l}\text { Distal portion of the } \\
\text { deltopectoral crest }\end{array}$ & I or II \\
\hline S. brevis & SCB & $\begin{array}{l}\text { Lateral coracoid, } \\
\text { above the } \mathrm{SCL}\end{array}$ & I' or II' & $\begin{array}{l}\text { Distal portion of the } \\
\text { deltopectoral crest }\end{array}$ & I or II \\
\hline $\begin{array}{l}\text { Coracobrachialis brevis } \\
\text { dorsalis }\end{array}$ & CBD & $\begin{array}{l}\text { Lateral surface of the } \\
\text { scapula, close to the } \\
\text { acromion }\end{array}$ & $I^{\prime}$ & $\begin{array}{l}\text { Proximolateral margin of } \\
\text { humerus, above the } \\
\text { deltopectoral crest }\end{array}$ & II' \\
\hline $\begin{array}{l}\text { Coracobrachialis brevis } \\
\text { ventralis }\end{array}$ & CBV & Lateral coracoid & $\mathrm{I}^{\prime}$ & $\begin{array}{l}\text { Internal surface of the } \\
\text { deltopectoral crest }\end{array}$ & $\mathrm{I}^{\prime}$ \\
\hline \multicolumn{6}{|l|}{ Triceps brachii } \\
\hline T. caput scapulare & TBS & $\begin{array}{l}\text { Caudolateral surface } \\
\text { of the glenoid rim, } \\
\text { on scar }\end{array}$ & I & Ulnar olecranon process & I \\
\hline $\begin{array}{c}\text { T. caput } \\
\text { coracoideus }\end{array}$ & TBC & $\begin{array}{l}\text { Ramii on the caudal } \\
\text { margin of scapula } \\
\text { and coracoid }\end{array}$ & II & Ulnar olecranon process & II \\
\hline T. lateralis & TBL & $\begin{array}{l}\text { Caudolateral surface } \\
\text { of humeral shaft }\end{array}$ & $I^{\prime}$ & Ulnar olecranon process & 1 \\
\hline T. caput mediale 1 & TBM1 & $\begin{array}{l}\text { Medial and distal } \\
\text { portion of the } \\
\text { humeral shaft }\end{array}$ & $\mathrm{I}^{\prime}$ & Ulnar olecranon process & I \\
\hline T. caput mediale 2 & TBM2 & $\begin{array}{l}\text { Caudomedial surface } \\
\text { of proximal humerus }\end{array}$ & $I^{\prime}$ & Ulnar olecranon process & I \\
\hline
\end{tabular}


T. caput mediale 3

TBM3

T. caput mediale $4 \quad$ TBM4

Biceps brachii

BB

Humeroradialis

HR

Brachialis

BR

Supinator

SU

Extensor carpi radialis

ECR

Extensor carpi ulnaris

ECU

Flexor ulnaris

FU

Abductor radialis

AR

Pronator teres

PT

Abductor pollicis

APL

longus

Extensor digitorum

longus

Extensor digiti I

superficialis

Extensor digiti I

profundus

Flexor digitorum brevis superficialis digiti I

Flexor digitorum profundus digiti I

Flexor digitorum longus

EDL

EDS

EDP

FDSI

FDPI

FDL
Caudal surface of th humeral shaft

Lateral and distal portion of the humeral shaft

Craniodorsal surface I' of the coracoid

Craniodorsal surface II' of humerus, caudal to the deltopectoral crest

Craniomedial surface I or I' of the humerus, distal to the deltopectoral crest, or on the cuboid fossa

Ectepicondyle of the humerus

\section{Ectepicondyle of the I} humerus

Ectepicondyle of the II humerus

Ectepicondyle of the I humerus

Ectepicondyle of the II humerus

Entepicondyle of the l' humerus

Lateral shaft of the l' radius and ulna
Ectepicondyle of the I humerus

Distal and anterior I' surface of radius and ulna and probably distal carpal I

Dorsolateral and dorsodistal surface of metacarpal I

Distal carpals

Distal carpals

Entepicondyle of the humerus, caudal surface of the ulna, and ulnar surface of distal carpals
Ulnar olecranon process II

Ulnar olecranon process II

Proximomedial surface of I the radius

Humeroradialis tubercle of II the proximal radius, on craniolateral side

Proximomedial surface of I' the radius

Cranial radial shaft I'

Dorsal surface of distal II' carpal I

Dorsolateral surface of $\quad$ II metacarpal II

Craniolateral surface of ulna I'

Cranial surface of the radius II'

Proximomedial surface of I' radius

Proximomedial margin of $\quad \mathrm{II}^{\prime}$ metacarpal I

Proximodorsal margin of I' metacarpal II

Extensor process of ungual I' phalanx

Extensor process of ungual I' phalanx

Flexor processes of phalanx I II

Flexor process of phalanx I I

Flexor surface of ungual I phalanges 
3 Levels of inference correspond to those that are conservative in extant archosaurs (I) or varied and thus

4 ambiguous for Archosauria (II); level III inferences (parsimoniously absent in ancestral Archosauria) were

5 not used. Prime ( $\left.I^{\prime}, I^{\prime}\right)$ annotations indicate attachments lacking clear osteological correlates, which can

6 still be reconstructed but only have approximate, relative rather than more specific, direct locations

7 (I,II). 


\section{Table 2 (on next page)}

Ranges of motion (ROMs) of each degree of freedom for Mussaurus and Crocodylus in the resting pose. 
1 Table 2. Ranges of motion (ROMs) of each degree of freedom for Mussaurus and Crocodylus in the resting pose.

\begin{tabular}{|c|c|c|c|c|c|c|c|c|c|c|}
\hline & Joint & $\begin{array}{l}\text { Pronation } \\
\left({ }^{\circ}\right)\end{array}$ & $\begin{array}{l}\text { Supination } \\
\left({ }^{\circ}\right)\end{array}$ & $\begin{array}{l}\text { Total long- } \\
\text { axis rotation } \\
\left({ }^{\circ}\right)\end{array}$ & $\begin{array}{l}\text { Abduction } \\
\left({ }^{\circ}\right)\end{array}$ & $\begin{array}{l}\text { Adduction } \\
\left({ }^{\circ}\right)\end{array}$ & $\begin{array}{l}\text { Total ab/ } \\
\text { adduction }\left({ }^{\circ}\right)\end{array}$ & $\begin{array}{l}\text { Flexion } \\
\left({ }^{\circ}\right)\end{array}$ & Extension $\left({ }^{\circ}\right)$ & $\begin{array}{l}\text { Total } \\
\text { flexion/ } \\
\text { extension } \\
\left({ }^{\circ}\right)\end{array}$ \\
\hline \multirow{5}{*}{$\begin{array}{l}\text { Mussaurus } \\
\text { patagonicus }\end{array}$} & Glenohumeral & -25 & 25 & 50 & -25 & 40 & 65 & -35 & -70 & 35 \\
\hline & Elbow & -30 & 0 & 30 & -5 & 5 & 10 & 130 & 20 & 110 \\
\hline & Wrist & - & - & - & -10 & 10 & 20 & 70 & -30 & 100 \\
\hline & $\begin{array}{l}\text { Metacarpo- } \\
\text { phalangeal }\end{array}$ & - & - & - & - & - & - & 50 & -40 & 90 \\
\hline & Interphalangeal & - & - & - & - & - & - & 70 & -25 & 95 \\
\hline \multirow{3}{*}{$\begin{array}{l}\text { Crocodylus } \\
\text { johnstoni }\end{array}$} & Glenohumeral & -20 & 20 & 40 & -5 & 45 & 50 & 5 & -60 & 65 \\
\hline & Elbow & -20 & 8 & 28 & -5 & 5 & 10 & 110 & 15 & 95 \\
\hline & Wrist & -10 & 30 & 40 & -30 & 5 & 35 & 40 & -60 & 100 \\
\hline
\end{tabular}

2 


\section{Table 3 (on next page)}

Muscle actions for the glenoid and elbow joints for Crocodylus and Mussaurus in the resting pose. 
Table 3. Muscle actions for the glenoid and elbow joints for Crocodylus and Mussaurus in the resting pose.

\begin{tabular}{|c|c|c|c|c|c|c|c|c|}
\hline \multirow[b]{3}{*}{ Muscle } & \multicolumn{6}{|c|}{ Glenoid } & \multicolumn{2}{|c|}{ Elbow } \\
\hline & \multicolumn{2}{|c|}{ Pronation/supination } & \multicolumn{2}{|c|}{ Extension/flexion } & \multicolumn{2}{|c|}{ Abduction/adduction } & \multicolumn{2}{|c|}{ Extension/flexion } \\
\hline & Crocodylus & Mussaurus & Crocodylus & Mussaurus & Crocodylus & Mussaurus & Crocodylus & Mussaurus \\
\hline DS & supination & supination & flexion & flexion & abduction & abduction & - & - \\
\hline DC & supination & supination & flexion & flexion & abduction & abduction & - & - \\
\hline $\mathrm{TM}$ & supination & supination & extension & extension & abduction & abduction & - & - \\
\hline SBS & pronation & pronation & extension & extension & adduction & adduction & - & - \\
\hline SHP & supination & pronation & extension & extension & abduction & abduction & - & - \\
\hline $\mathrm{SCl}$ & supination & supination & flexion & flexion & adduction & adduction & - & - \\
\hline SCB & supination & supination & flexion & flexion & adduction & adduction & - & - \\
\hline $\mathrm{SCL}$ & supination & supination & flexion & flexion & adduction & adduction & - & - \\
\hline CBV & pronation & pronation & extension & flexion & adduction & adduction & - & - \\
\hline CBD & mixed & supination & flexion & flexion & adduction & mixed & - & - \\
\hline TBS & supination & mixed & extension & mixed & abduction & abduction & extension & extension \\
\hline TBC & supination & supination & extension & extension & abduction & abduction & extension & extension \\
\hline TBM4 & - & - & - & - & - & - & extension & extension \\
\hline TBM1 & - & - & - & - & - & - & extension & extension \\
\hline TBM3 & - & - & - & - & - & - & extension & extension \\
\hline TBL & - & - & - & - & - & - & extension & extension \\
\hline TBM2 & - & - & - & - & - & - & extension & extension \\
\hline $\mathrm{BB}$ & supination & mixed & flexion & flexion & adduction & adduction & flexion & flexion \\
\hline HR & - & - & - & - & - & - & flexion & flexion \\
\hline$B R$ & - & - & - & - & - & - & flexion & flexion \\
\hline SU & - & - & - & - & - & - & extension & mixed \\
\hline FU & - & - & - & - & - & - & extension & mixed \\
\hline$A R$ & - & - & - & - & - & - & extension & mixed \\
\hline PT & - & - & - & - & - & - & extension & flexion \\
\hline FDL & - & - & - & - & - & - & mixed & flexion \\
\hline EDL & - & - & - & - & - & - & extension & mixed \\
\hline ECR & - & - & - & - & - & - & extension & mixed \\
\hline ECU & - & - & - & - & - & - & extension & mixed \\
\hline
\end{tabular}

2 Bold font highlights a difference between the two taxa. ' - ' indicates that the muscle was inferred not to act around that axis in the model. 


\section{Table 4(on next page)}

Muscle actions for the wrist and manus of Crocodylus and Mussaurus. 
1 Table 4. Muscle actions for the wrist and manus of Crocodylus and Mussaurus.

2

\begin{tabular}{|c|c|c|c|c|}
\hline & \multicolumn{2}{|c|}{ Wrist } & \multirow{2}{*}{$\begin{array}{c}\text { Metacarpo-phalangeal } \\
\text { Extension/flexion }\end{array}$} & \multirow{3}{*}{$\begin{array}{c}\text { Interphalangeal } \\
\text { Extension/flexion } \\
\text { Mussaurus }\end{array}$} \\
\hline & Extensi & flexion & & \\
\hline Muscle & Crocodylus & Mussaurus & Mussaurus & \\
\hline ECR & extension & extension & - & - \\
\hline APL & extension & extension & - & - \\
\hline EDL & extension & extension & - & - \\
\hline ECU & extension & extension & - & - \\
\hline EDS & - & - & extension & extension \\
\hline EDP & - & - & extension & extension \\
\hline FDL & flexion & flexion & flexion & flexion \\
\hline FDSI & - & - & flexion & - \\
\hline FDPI & - & - & flexion & - \\
\hline
\end{tabular}

3 ' - ' indicates that the muscle was inferred not to act around that axis in the model; or was not modelled

4 in that regard. 